\title{
Dichotomies for Queries with Negation in Probabilistic Databases
}

\author{
ROBERT FINK and DAN OLTEANU, University of Oxford
}

\begin{abstract}
This article charts the tractability frontier of two classes of relational algebra queries in tuple-independent probabilistic databases. The first class consists of queries with join, projection, selection, and negation, but without repeating relation symbols and union. The second class consists of quantified queries that express the following binary relationships amongst sets of entities: set division, set inclusion, set equivalence, and set incomparability. Quantified queries are expressible in relational algebra using join, projection, nested negation, and repeating relation symbols.

Each query in the two classes has either polynomial-time or \#P-hard data complexity and the tractable queries can be recognised efficiently. Our result for the first query class extends a known dichotomy for conjunctive queries without self-joins to such queries with negation. For quantified queries, their tractability is sensitive to their outermost projection operator: They are tractable if no attribute representing set identifiers is projected away and \#P-hard otherwise.
\end{abstract}

Categories and Subject Descriptors: H.2.4 [Database Management]: Query Processing; G.2.1 [Combinatorics]: Counting Problems

General Terms: Algorithms, Theory

Additional Key Words and Phrases: complexity dichotomy, knowledge compilation, probabilistic databases

ACM Reference Format:

Robert Fink and Dan Olteanu. 2015. Dichotomies for queries with negation in probabilistic databases. ACM Trans. Datab. Syst. 1, 1, Article 1 (October 2015), 45 pages.

DOI : http://dx.doi.org/10.1145/0000000.0000000

\section{INTRODUCTION}

Charting the tractability frontier of query evaluation lies at the foundation of probabilistic databases. The probabilistic database systems MystiQ Dalvi and Suciu 2007a and MayBMS/SPROUT [Huang et al. 2009] distinguish between tractable and intractable queries at compile time and provide exact evaluation techniques for tractable queries at the speed of relational databases and approximate techniques for intractable queries. The relevance of such tractability results goes beyond probabilistic databases: the problems of tractable query evaluation in probabilistic databases and of domain-lifted inference for weighted first-order model counting den Broeck 2011, which is actively investigated by the AI community, essentially coincide Gribkoff et al. 2014b.

Complexity dichotomies have been established for several classes of relational queries in probabilistic databases: Any query in such a class is either tractable or intractable, that is, its data complexity is either polynomial time or \#P-hard. Such dichotomies are known for conjunctive queries without repeating relation symbols Dalvi and Suciu 2007a and their extension to ranking [Olteanu and Wen 2012], and for unions of conjunctive queries Dalvi] and Suciu 2012. This article complements these results with dichotomies for two classes of queries with negation. These dichotomies has been announced previously in extended abstracts Fink et al. 2011, Fink and Olteanu 2014].

Permission to make digital or hard copies of part or all of this work for personal or classroom use is granted without fee provided that copies are not made or distributed for profit or commercial advantage and that copies show this notice on the first page or initial screen of a display along with the full citation. Copyrights for components of this work owned by others than ACM must be honored. Abstracting with credit is permitted. To copy otherwise, to republish, to post on servers, to redistribute to lists, or to use any component of this work in other works requires prior specific permission and/or a fee. Permissions may be requested from Publications Dept., ACM, Inc., 2 Penn Plaza, Suite 701, New York, NY 10121-0701 USA, fax +1 (212) 869-0481, or permissions@acm.org.

(C) 2015 ACM 0362-5915/2015/10-ART1 $\$ 15.00$

DOI : http://dx.doi.org/10.1145/0000000.0000000 
The two query classes considered in this article are fragments of relational algebra without union. The first class is denoted $1 \mathrm{RA}^{-}$and consists of queries with equi-join, projection, selection, and negation, which is expressed using the difference operator, but without repeating relation symbols. Examples of $1 \mathrm{RA}^{-}$queries are given in Figs. 1 and 3 The second class consists of the set-division, set-inclusion, set-equivalence, set-incomparability quantified queries. In contrast to $1 \mathrm{RA}^{-}$queries, quantified queries can only be expressed in relational algebra using repeating relation symbols, cf. Figs. 5 and 15 The two classes can also be mixed by allowing $1 \mathrm{RA}^{-}$queries in place of relations in quantified queries.

The tractable queries from both classes admit efficient syntactic recognition. This is convenient for practical reasons: By just inspecting the query, the query optimiser of a probabilistic database management system can quickly decide whether to employ efficient exact inference in case of tractable queries, or approximate inference otherwise.

The probabilistic database model considered in this article is that of tuple-independent databases, where each tuple in the database is an independent probabilistic event. Prime examples of tuple-independent probabilistic databases are the knowledge bases from Google Knowledge Vault Dong et al. 2014 and NELL Carlson et al. 2010]. For more complex probabilistic models, query tractability is quickly lost: for block-independent disjoint tables consisting of independent groups of mutually exclusive tuples, tractability essentially falls back to that for tuple-independent databases by restricting joins to attributes representing group keys, while for the general model of probabilistic c-tables, simple selection and projection queries can be \#P-hard Suciu et al. 2011].

The following theorem states our first dichotomy result:

Theorem 1.1. The data complexity of any $1 R A^{-}$query $Q$ on tuple-independent databases is polynomial time if $Q$ is hierarchical and \#P-hard otherwise.

We next define the hierarchical property for a $1 \mathrm{RA}^{-}$query $Q$. We denote by $[A]$ the equivalence class of attribute $A$ in $Q$, as enforced by join and difference operators; for instance, given relations over schemas $X\left(A_{1}\right)$ and $Y\left(A_{2}\right)$, both the join $X \bowtie_{A_{1}=A_{2}} Y$ and the difference $X-{ }_{A_{1} \leftrightarrow A_{2}} Y$ under the attribute mapping $A_{1} \leftrightarrow A_{2}$ enforce that $\left[A_{1}\right]=\left[A_{2}\right]$.

Definition 1.2. A $1 \mathrm{RA}^{-}$query $Q$ is hierarchical if for every pair of attribute classes $[A]$ and $[B]$ without attributes in $Q$ 's result or in selections with equality conditions, there is no triple of relation symbols $R, S$, and $T$ in $Q$ such that $R$ has attributes in $[A]$ and not in $[B], S$ has attributes in both $[A]$ and $[B]$, and $T$ has attributes in $[B]$ and not in $[A]$.

Example 1.3. Figure 1 depicts the non-hierarchical query $\pi_{\emptyset}\left[X \bowtie\left(R-\pi_{A}(T \bowtie S)\right)\right]$ over schema $(X(A), R(A), T(B), S(A, B))$. The query $\pi_{\emptyset}(R \bowtie S \bowtie T)$ over the same schema is the classical example of a non-hierarchical query without negation [Suciu et al. 2011. NonBoolean versions of these queries are hierarchical. Figure 3 depicts the hierarchical query $\pi_{\emptyset}[R \times T-U \times V]$ over schema $(R(A), U(A), T(B), V(B))$.

Hierarchical $1 \mathrm{RA}^{-}$queries can be recognised in LOGSPACE. The hierarchical property plays a central role in studies with seemingly disparate focus. All join queries that admit parallel evaluation with one broadcast step in the Massively Parallel Communication model are hierarchical Koutris and Suciu 2011]. The results of any hierarchical conjunctive query in relational databases admit lossless factorised representations that are at most linear in the size of the input databases Olteanu and Závodný 2015. In the finite cursor model, the hierarchical queries are exactly those semi-join algebra queries with one-step streaming evaluation Grohe et al. 2009. In provenance databases, the hierarchical queries are exactly those conjunctive queries with provenance polynomials of bounded readability Olteanu and Závodný 2012. The hierarchical queries are exactly those non-repeating conjunctive queries that are tractable in probabilistic databases Dalvi and Suciu 2007a. A key contribution of 


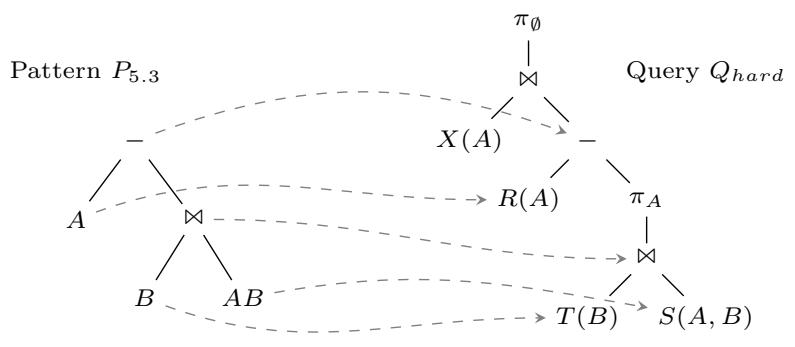

Fig. 1. Query pattern $P_{5.3}$ (left) matched by the $1 \mathrm{RA}{ }^{-}$query $Q_{\text {hard }}$ (right).

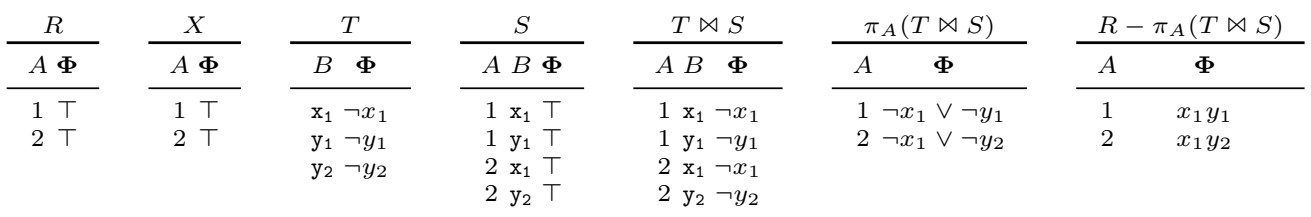

Fig. 2. Hardness reduction for query $Q_{\text {hard }}$ in Fig. 1 and formula $x_{1} y_{1} \vee x_{1} y_{2}$. To avoid clutter (and in contrast to Sec. 5 s naming convention), relations may share attribute names and all joins are natural.

this article is to understand the connection between the hierarchical property and negation in probabilistic databases. Theorem 1.1 states that the hierarchical property partitions the query language $1 \mathrm{RA}^{-}$into tractable and hard queries, thereby lifting the dichotomy for non-repeating conjunctive queries Dalvi and Suciu 2007a to queries with negation. In Section 7. we discuss difficulties of extending this result to non-repeating relational calculus with negation and to non-repeating relational algebra with union.

The tractability and hardness proofs for $1 \mathrm{RA}^{-}$are non-trivial generalisations of those for queries without negation. Careful treatment is needed for the interaction of projection and difference operators, which can encode universal quantification and can lead to hardness already for cases where one input relation is probabilistic and all other relations are deterministic. A further source of complexity is the lack of commutativity and associativity of the difference operator, which leads to many incomparable minimal hard query patterns defined by the interaction between difference and join operators. In contrast, for queries without negation there is a single minimal hard pattern and it requires two probabilistic relations. We next exemplify techniques used in the hardness and tractability proofs.

\section{\#P-hardness of non-hierarchical queries}

We prove that every non-hierarchical $1 \mathrm{RA}^{-}$query $Q$ has \#P-hard data complexity by reduction from the \#P-hard model-counting problem for positive bipartite DNF formulas: Given such a formula $\Psi$ and the query $Q$, for most reductions used in this article we construct an input database whose input tuples are annotated with variables in $\Psi$ such that the result of $Q$ becomes annotated with $\Psi$ or $\neg \Psi$. To count the models of $\Psi$, we call an oracle that computes the probability $P_{Q}$ of the query $Q$ on a tuple-independent database where each variable has probability $1 / 2$. The number of models $\# \Psi$ is then $2^{n} P_{Q}$ or $2^{n}\left(1-P_{Q}\right)$, where $n$ is the number of variables in $\Psi$. The query evaluation problem is not technically in \#P since it is not a counting problem, cf. [Suciu et al. 2011] (page 47) for a detailed discussion.

The starting point of our analysis is an alternative characterisation of the hierarchical property via minimal hard patterns: A query is not hierarchical exactly when it matches such a pattern Fink and Olteanu 2014. There is a pattern for each possible binary tree with leaves $A, A \bar{B}$, and $B$, and with inner nodes join and difference operators (48 in total). 
A query matches a pattern if there is a total mapping of the nodes of the pattern to nodes in the parse tree of the query such that: (1) The join and difference operators in the pattern are mapped to join and respectively difference operators in the query; (2) the leaves $A$, $A B$, and $B$ are mapped to relations $R, S$, and respectively $T$ as in Definition 1.2 and (3) parent-child edges in the pattern are mapped to ancestor-descendant edges in the query.

Example 1.4. For each query, we craft a specific reduction depending on which pattern is matched. For example, the query $Q_{\text {hard }}$ in Fig. 1 (right) is not hierarchical as it matches the pattern in Fig. 1 (left). We exemplify the reduction for formula $\Psi=x_{1} y_{1} \vee x_{1} y_{2}$, where we consider each variable random and with probability $1 / 2$.

We populate the relations $R, S, T$, and $X$ as shown in Fig. 2 The relations $R$ and $X$ consist of tuples representing the indices 1 and 2 of the clauses in $\Psi$ and annotated by the Boolean constant true $(T)$. The relation $S$ lists all pairs of the index of a clause and variable in that clause; these tuples are also annotated by $T$. Finally, the relation $T$ lists all variables occurring in $\Psi$, where each tuple for variable $z$ is annotated by $\neg z$. In our encoding, we may use variable names as constants, e.g., the values of the attribute $B$ in relations $R$ and $T$.

The probabilistic database $(R, X, S, T)$ represents a finite set of possible database instances, with each instance defined by a total assignment of the variables in the annotation columns $\Phi$ Suciu et al. 2011. The instance defined by a variable assignment consists of those tuples whose annotations are satisfied by the assignment. For instance, the assignment mapping all variables $x_{1}, y_{1}$, and $y_{2}$ to $T$ defines the following database instance: $R$, $S$ and $X$ retain all their tuples, since their annotation $T$ is always satisfied; and $T$ becomes empty, since the assignment falsifies the annotation of each tuple in $T$.

Figure 2 also depicts the intermediate results during the computation of $Q_{\text {hard }}$. Whereas the input relations are tuple-independent, the intermediate results exhibit correlated annotations. These annotations are Boolean formulas over the annotations of the input relations Green et al. 2007): a join (projection) of tuples is annotated by the conjunction (respectively, disjunction) of their annotations, and a difference of two tuples is the conjunction of the annotation of the first tuple and the negation of the second tuple. The query result is the projection on the empty set of the bottom-right relation; the annotation associated with this nullary result tuple is $\Psi$.

Example 1.4 shows the power of negation: Our query $Q_{\text {hard }}$ can compute \# $\Psi$ for any positive $2 \mathrm{DNF}$ formula $\Psi$ and is thus \#P-hard already when one of its relations is probabilistic (here, $T$ ) while all other relations are deterministic. In contrast, hardness can only be achieved for queries without negation when at least two input relations are probabilistic.

The key challenge in the hardness reductions from Section 5 is to identify three relations $(R, S, T)$ that establish the match of a non-hierarchical query with one of the minimal hard patterns, and to populate them such that the annotation of the query result is the input positive $2 \mathrm{DNF}$ formula $\Psi$. The remaining relations ( $X$ in Example 1.4 ) are populated such that they do not influence the interaction between the annotations of $R, S$, and $T$. The reductions put forward in this article vary substantially and there is no one unifying reduction for all the minimal hard patterns. Indeed, some reductions require a single relation to be probabilistic, while others require two. Furthermore, some reductions populate a single probabilistic database (such as in the above example), while others require to populate a number of databases linear in the size of the input formula $\Psi$.

\section{Efficient evaluation algorithm for hierarchical queries}

We evaluate a hierarchical $1 \mathrm{RA}^{-}$query $Q$ in five steps: (1) We translate $Q$ into an equivalent relational calculus expression $Q_{R C}$ that is further rewritten into a disjunction of disjunctionfree existential relational calculus expressions by pushing down negation and existential quantifiers; (2) we compute the formulas representing the annotations of the results of $Q_{R C}$ 's disjuncts; (3) we compile each such formula into an ordered binary decision diagram 


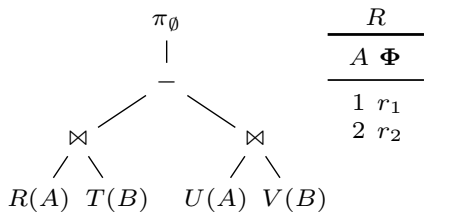

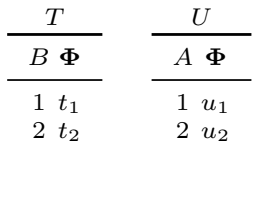

\begin{tabular}{cc}
$\frac{V}{B}$ & $\mathbf{\Phi}$ \\
\hline 1 & $v_{1}$ \\
2 & $v_{2}$
\end{tabular}

\begin{tabular}{ccc}
\multicolumn{3}{c}{$R \bowtie T$} \\
\hline$A$ & $B$ & $\mathbf{\Phi}$ \\
\hline 1 & 1 & $r_{1} t_{1}$ \\
1 & 2 & $r_{1} t_{2}$ \\
2 & 1 & $r_{2} t_{1}$ \\
2 & 2 & $r_{2} t_{2}$
\end{tabular}

\begin{tabular}{ccc}
\multicolumn{3}{c}{$R \bowtie T-U \bowtie V$} \\
\hline$A$ & $B$ & $\mathbf{\Phi}$ \\
\hline 1 & 1 & $r_{1} t_{1} \neg\left(u_{1} v_{1}\right)$ \\
1 & 2 & $r_{1} t_{2} \neg\left(u_{1} v_{2}\right)$ \\
2 & 1 & $r_{2} t_{1} \neg\left(u_{2} v_{1}\right)$ \\
2 & 2 & $r_{2} t_{2} \neg\left(u_{2} v_{2}\right)$
\end{tabular}

Fig. 3. Hierarchical $1 \mathrm{RA}^{-}$Boolean query $Q_{\text {easy }}$ and a database $\mathcal{D}=(R, T, U, V)$. The tables $R \bowtie T$ and $R \bowtie T-U \bowtie V$ show how the annotations of $R, T, U, V$ are propagated by $Q_{e a s y}$.

(OBDD); (4) we compute an OBDD representing the disjunction of the OBDDs from step (3); (5) and finally, we compute the probability of the OBDD from step (4). Resorting to OBDDs for query evaluation in probabilistic databases is not new Olteanu and Huang 2008 Jha and Suciu 2013. While for arbitrary queries the OBDDs may be exponential in the size of their annotations and thus of the input database, we show in Section 3 that those from step (5) can be computed in time polynomial in the input database. Since OBDDs admit linear-time probability computation [Wegener 2004], we obtain an overall query evaluation algorithm with polynomial-time data complexity. While the OBDD sizes are independent of the query size and linear in the database size for hierarchical non-repeating conjunctive queries Olteanu and Huang 2008, they remain linear in the database size, but may depend exponentially on the query size, for hierarchical $1 \mathrm{RA}^{-}$queries. The exponential dependency on the query size arises due to the query rewriting and the OBDD construction steps.

Example 1.5. Consider the hierarchical query $Q_{\text {easy }}$ and the database $\mathcal{D}$ from Fig. 3 The formula annotating $Q_{\text {easy }}$ 's result is

$$
\Psi=r_{1}\left[t_{1}\left(\neg u_{1} \vee \neg v_{1}\right) \vee t_{2}\left(\neg u_{1} \vee \neg v_{2}\right)\right] \vee r_{2}\left[t_{1}\left(\neg u_{2} \vee \neg v_{1}\right) \vee t_{2}\left(\neg u_{2} \vee \neg v_{2}\right)\right] .
$$

The difference operator entangles the annotations of the participating relations in such a way that the resulting annotation $\Psi$ is not a read-once formula, that is, a formula where each variable appears once; this entanglement is the pivotal intricacy introduced by the difference operator.

We show in Section 3 that for every tuple-independent database $\mathcal{D}$, the annotation of the result of $Q_{\text {easy }}$ on $\mathcal{D}$ admits an OBDD of size $\mathcal{O}\left(|\mathcal{D}| \cdot f\left(Q_{\text {easy }}\right)\right)$, where $f\left(Q_{\text {easy }}\right)$ is the OBDD width (cf. Section 2.3) and only depends on the query size $\left|Q_{e a s y}\right|$. The underlying idea is to translate $Q_{\text {easy }}$ into an equivalent disjunction of disjunction-free existential relational calculus queries such that each of the disjuncts gives rise to a compact OBDD and all OBDDs have compatible variable orders and can be combined efficiently into a single OBDD. We denote the language of such queries by $\mathrm{RC}^{\exists}$. For $Q_{\text {easy }}$, this translation yields the query

$$
Q_{R C}=\underbrace{\exists_{A}(R(A) \wedge \neg U(A)) \wedge \exists_{B} T(B)}_{Q_{1}} \vee \underbrace{\exists_{A} R(A) \wedge \exists_{B}(T(B) \wedge \neg V(B))}_{Q_{2}} .
$$

The formulas annotating the results of $Q_{1}$ and $Q_{2}$ on the database $\mathcal{D}$ from Fig. 3 are

$$
\Psi_{1}=\left(r_{1} \neg u_{1} \vee r_{2} \neg u_{2}\right) \wedge\left(t_{1} \vee t_{2}\right) \quad \Psi_{2}=\left(r_{1} \vee r_{2}\right) \wedge\left(t_{1} \neg v_{1} \vee t_{2} \neg v_{2}\right) .
$$

and clearly $\Psi_{1} \vee \Psi_{2} \equiv \Psi$. The queries $Q_{1}$ and $Q_{2}$ can be written such that (i) for each quantifier $\exists_{X}(Q)$ every relation symbol in $Q$ contains the variable $X$, and (ii) the nesting order of the quantifiers is the same in both $Q_{1}$ and $Q_{2}$. Property (i) ensures that the formulas $\Psi_{1}$ and $\Psi_{2}$ admit OBDDs of size $\mathcal{O}(|\mathcal{D}|)$, as exemplified in the diagrams of Fig. 4 Property (ii) implies that these OBDDs have the same global variable order, which enables efficient computation of their conjunctions, disjunctions, and negation Wegener 2004]. 


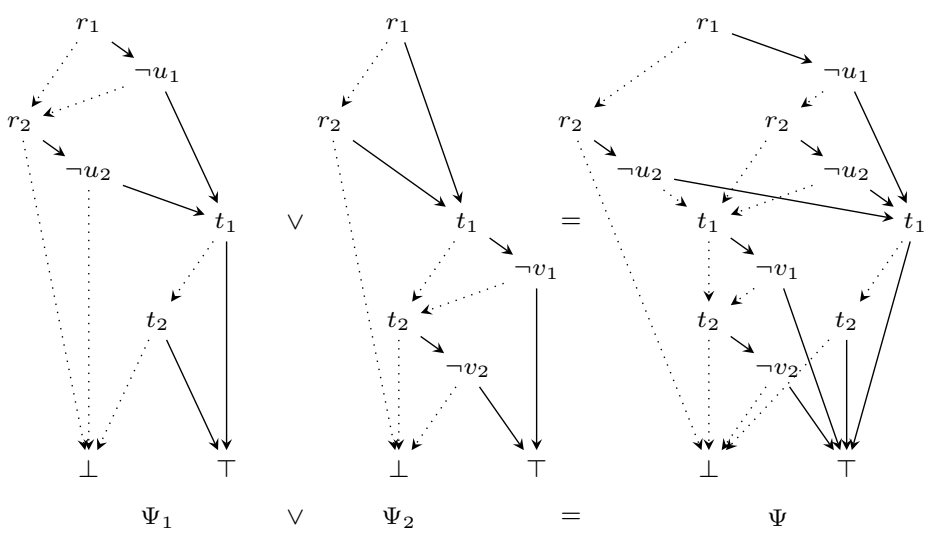

Fig. 4. From left to right: OBDDs for $\Psi_{1}, \Psi_{2}$, and $\Psi=\Psi_{1} \vee \Psi_{2}$ in Example 1.5 The inner nodes of the OBDD are variables in $\Psi$ and the leaves are the Boolean constants $\top$ and $\perp$. For an inner node $n$, the outgoing dotted edge is for $n=\perp$, while the outgoing solid edge is for $n=T$. The three OBDDs share the same order of variables on any root-to-leaf path.

\section{Quantified queries for reasoning about sets}

The study of tractability for queries with repeating relation symbols raises additional challenges due to the interaction between copies of the same relation and to query containment. The language of unions of conjunctive queries (with repeating relation symbols) admits a complexity dichotomy, though there is no syntactic characterisation of tractable queries in this language; instead, there is an algorithm that runs in polynomial time for all tractable unions of conjunctive queries Dalvi and Suciu 2012.

In Section 6]we investigate the class of so-called quantified queries that are expressible in an extension of $1 \mathrm{RA}^{-}$queries with repeating relation symbols. They express binary relationships amongst sets of items encoded in relations. We consider set division, set inclusion, set equality, set difference, and set incomparability. Their definitions in relational algebra are given in Figs. 5 and 15 . Each quantified query stands for a set of queries if we allow the input relations to be replaced by hierarchical $1 \mathrm{RA}^{-}$queries whose results are tuple-independent probabilistic relations.

Given a relation $S$ (sid, item) encoding an arbitrary number of sets and their items, the set inclusion query $S_{\subseteq}$ returns the pairs of set identifiers $\operatorname{sid}_{1}$ and $\operatorname{sid}_{2}$ such that all items of $\operatorname{sid}_{1}$ are also items of $\operatorname{sid}_{2}$; the result of the set equality query $S_{=}$consists of those pairs of sets that consist of the same items; the result of the set incomparability query $S_{<>}$ consists of those pairs of sets such that none is contained in the other. Given the above relation $S$ (sid,item) and a second relation $I$ (item) consisting of items, the set division query $S \div I$ returns those sets that contain all items in $I$. Such queries are used in decision support applications, such as insurance and healthcare Rao et al. 1996 or data mining applications Rantzau 2004.

Similar to $1 \mathrm{RA}^{-}$queries, the tractable quantified queries admit efficient syntactic recognition. In particular, the queries are tractable if they retain in the result the attributes for set identifiers. If some of these attributes are projected away, then they become \#P-hard.

Example 1.6. Consider a tuple-independent database consisting of a supplier relation $S$ with two columns sid for supplier key and pid for product key, and a product relation $I$ with only a product item key pid that contains keys of all product items of a given brand (cf. Fig. 5). Then the set division query $S \div P$ returns suppliers that supply all products of this brand. The query can be expressed as shown in Fig. 5 . In a deterministic setting, the result would only consist of the tuple with sid 1 , since this is the only sid paired in $S$ with 


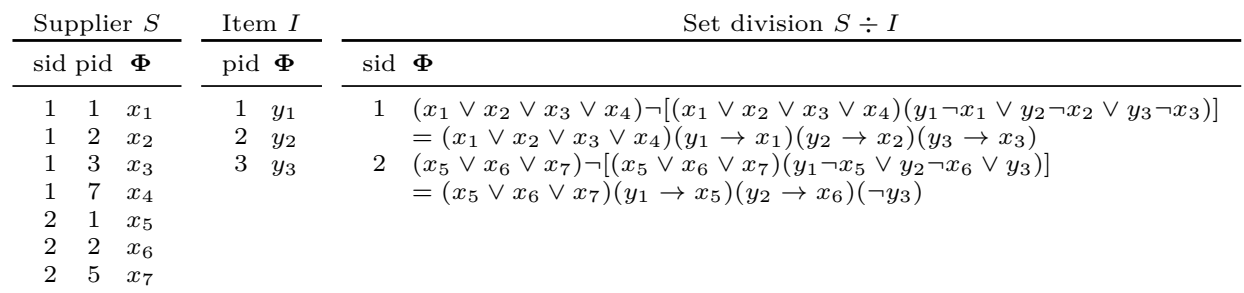

Fig. 5. Supplier $S$, Item $I$, Set division $S \div I=\pi_{\text {sid }}(S)-\pi_{\text {sid }}\left(\pi_{\text {sid }}(S) \bowtie I-S\right)$.

all pids in $I$. In a probabilistic setting, the third tuple from $I$ can be absent with probability $1-P_{y_{3}}$, where $P_{y_{3}}$ is the probability that this tuple is present. In that case, sid 2 can be in the result of $S \div I$, since sid 2 is paired in $S$ with pids 1 and 2 but not with 3 . The set of instances that witness sid $\in\{1,2\}$ in the result is defined by the annotation expressions over the input random variables given in column $\Phi$.

We explain the annotation for sid 1 by following the structure of the query. The projection $\pi_{\text {sid }}(S)$ corresponds to the formula $x_{1} \vee \cdots \vee x_{4}$ : Indeed, sid 1 can be part of the result if at least one of these variables is true. The subsequent cross product with $I$ generates the tuple $(1, i)$ with annotation $\left(x_{1} \vee \cdots \vee x_{4}\right) y_{i}$ for $1 \leq i \leq 3$. The difference with $S$ keeps all tuples in the product and their annotations are extended by the negation of the annotation of the corresponding tuples in $S$ : In case of tuple $(1, i)$, its annotation becomes $\left(x_{1} \vee \cdots \vee x_{4}\right) y_{i} \neg x_{i}$. The next projection yields the disjunction of all annotations for tuples $(1,1),(1,2)$, and $(1,3):\left(x_{1} \vee \cdots \vee x_{4}\right)\left[y_{1} \neg x_{1} \vee y_{2} \neg x_{2} \vee y_{3} \neg x_{3}\right]$. The outermost difference produces the annotation in Fig. 5 . The final annotation reads as follows: sid 1 is in the result provided at least one of the tuples with sid 1 is present in $S$, and if pid $=i$ is present in $I$, then the tuple $(1, i)$ must be present in $S$. We show in Section 6 that the probability of this annotation can be computed in time linear in the size of the input database. However, the Boolean version of the division query, namely $\pi_{\emptyset}(S \div I)$ is \#P-hard.

\section{Organisation of the article}

The remainder of the article is organised as follows. Section 2 recalls background on propositional formulas and probabilistic databases. Section 3 presents the polynomial-time procedure for computing probabilities of hierarchical 1RA queries. Section 4 introduces techniques necessary in the hardness proofs for non-hierarchical queries, and Section 5 details the \#P-hardness reductions. The case of quantified queries is discussed in Section 6] The article closes with a discussion of related work and open research directions in Section 7

\section{PRELIMINARIES}

We introduce necessary vocabulary for the $1 \mathrm{RA}^{-}$query language, the $\mathrm{RC}^{\exists}$ relational calculus to which we translate $1 \mathrm{RA}^{-}$queries for query evaluation, probabilistic databases, propositional formulas annotating results of queries in probabilistic databases, and their compilation into ordered binary decision diagrams as used for efficient query evaluation.

\subsection{The relational algebra subset $1 \mathbf{R A}^{-}$}

We assume database schemas with unique attribute names. The set of attributes of a relation $R$ is $\operatorname{sch}(R)$. A query $Q$ is non-repeating if each relation symbol occurs at most once in $Q$.

The relational algebra subset $1 \mathrm{RA}^{-}$consists of queries composed of:

- Non-repeating relation symbols;

- Equi-join: $Q_{1} \bowtie_{\rho} Q_{2}$, where $\rho$ is a conjunction of equality conditions $\rho=\left(A_{1}=B_{1}\right) \wedge \cdots \wedge$ $\left(A_{n}=B_{n}\right)$ such that all $A_{i}$ are attributes of $Q_{1}$ and all $B_{i}$ are attributes of $Q_{2}$; 
- Projection: $\pi_{A_{1}, \ldots, A_{n}}$ for attributes $A_{1}, \ldots, A_{n}$. We also use $\pi_{-B_{1}, \ldots,-B_{m}}(Q)=\pi_{-[B]}(Q)$ as shorthand for discarding the attributes in the class $[B]=\left\{B_{1}, \ldots, B_{m}\right\}$;

- Difference: $Q_{1}-{ }_{\rho} Q_{2}$, where $Q_{1}$ and $Q_{2}$ 's results are over schemas $\left\{A_{1}, \ldots, A_{n}\right\}$ and $\left\{B_{1}, \ldots, B_{n}\right\}$ respectively, and $\rho$ is the attribute mapping $\left(A_{1} \leftrightarrow B_{1}\right) \wedge \cdots \wedge\left(A_{n} \leftrightarrow B_{n}\right)$;

- Selection: $\sigma_{A \theta c}$, where $A$ is an attribute, $c$ a constant, and $\theta$ an arithmetic comparison.

Without loss of generality, we only consider in the sequel 1RA ${ }^{-}$queries without selections: Selections can be resolved prior to the development put forward in this article, since their results on tuple-independent relations are also tuple-independent. We recall from Definition 1.2 that in case of an equality condition $A=c$, we can safely ignore the attribute class $[A]$ when checking the hierarchical property since $A$ can only take one value.

In $Q_{1} \bowtie_{\rho} Q_{2}$ and $Q_{1}-{ }_{\rho} Q_{2}$, we write $A \in \rho$ to express that $\rho$ contains an equality or mapping on $A$, and $\left(A=A^{\prime}\right) \in \rho$ or $\left(A \leftrightarrow A^{\prime}\right) \in \rho$ to express that $\rho$ contains the equality $A=A^{\prime}$ or mapping $A \leftrightarrow A^{\prime}$, respectively. When no confusion arises, we choose a schema with suggestive unique attribute names like $R\left(A_{r}\right), S\left(A_{s}, B_{s}\right), T\left(B_{t}\right)$ and then write the queries $R \bowtie_{A_{r}=A_{s}} S$ and $(R \bowtie T)-A_{r} \leftrightarrow A_{s} \wedge B_{t} \leftrightarrow B_{s} S$ more concisely as $R \bowtie S$ and $(R \bowtie T)-S$.

We interchangeably use algebraic expressions and their ordered parse trees when referring to queries; in the latter case, the leaves are relations and inner nodes are algebra operators. Given a query $Q$ and an operator $O p$ in $Q, O p$ has even polarity if the number of "-" operators between $O p$ (exclusive) and the root of $Q$ (inclusive), for which $O p$ is a right descendant, is even, and has odd polarity otherwise. The pol function captures this notion: $\operatorname{pol}(Q, O p)$ is 1 if $O p$ has odd polarity in $Q$, and 0 otherwise. The bottom join operator in $Q_{\text {hard }}$ from Fig. 1 has polarity 1; for query $Q$ in Fig. 7 the joins on the leftmost path in have polarity 0 , the join of $V$ and $X$ has polarity 1, and relation $S$ has polarity 2 .

The equivalence class $[A]$ of an attribute $A$ in $Q$ consists of $A$ and all attributes made equal or mapped to $A$ in $Q$.

The attributes exported by a query $Q$, denoted $\mathcal{E}(Q)$, are defined on the query structure:

$$
\begin{aligned}
& \mathcal{E}\left(Q_{1} \bowtie_{\rho} Q_{2}\right)=\mathcal{E}\left(Q_{1}\right) \cup \mathcal{E}\left(Q_{2}\right) \quad \mathcal{E}\left(Q_{1}-{ }_{\rho} Q_{2}\right)=\mathcal{E}\left(Q_{1}\right) \\
& \mathcal{E}\left(\pi_{A_{1}, \ldots, A_{n}}(Q)\right)=\left\{A_{1}, \ldots, A_{n}\right\} \quad \mathcal{E}\left(\pi_{-[B]}(Q)\right)=\mathcal{E}(Q)-[B] \quad \mathcal{E}(R)=\operatorname{sch}(R) .
\end{aligned}
$$

A query $Q$ exports $[A]$ if there exists $A^{\prime} \in[A]$ such that $A^{\prime} \in \mathcal{E}(Q)$. Conversely, $Q$ does not export $[A]$ if for all $A^{\prime} \in[A]$ it holds that $A^{\prime} \notin \mathcal{E}(Q)$. By $Q^{[A]}, Q^{[\neg B]}$, and $Q^{[A][\neg B]}$ we denote a query $Q$ that exports $[A]$, does not export $[B]$, and respectively exports $[A]$ and not $[B]$. Using this notation, the triple of relations used to disprove the hierarchical property in Definition 1.2 is $\left(R^{[A][\neg B]}, T^{[B][\neg A]}, S^{[A][B]}\right)$ for distinct attributes $A$ and $B$.

\subsection{The relational calculus subset $\mathrm{RC}^{\exists}$}

Our query tractability results in Section 3 make use of standard translation between the relational algebra subset $1 \mathrm{RA}^{-}$and the relational calculus subset called $\mathrm{RC}^{\exists}$. The latter consists of queries $\{H \mid F\}$, where the query head $H$ is the set of query variables that occur unquantified in the query body $F$, and $F$ is a formula defined by the following grammar:

$$
F::=R\left(X_{1}, \ldots, X_{n}\right)\left|\exists_{X}\left(F_{1}\right)\right| F_{1} \wedge F_{2}\left|F_{1} \vee F_{2}\right| \neg\left(F_{1}\right),
$$

The size $|Q|$ of a query $Q$ is the number of its relation symbols.

\subsection{Propositional formulas and their compilation into decision diagrams}

Propositional formulas are essential to the probabilistic database formalism used in this article. We next review the necessary vocabulary from their syntax to (probabilistic) semantics, and conclude with their compilation to ordered binary decision diagrams.

Syntax. Let $\mathbf{X}$ be a finite set of variable symbols. A literal is a variable or its negation. A clause is a conjunction of literals. A formula can be constructed using variables and 
constants $\top$ (true) and $\perp$ (false) using the logical connectives $\vee$ (or), $\wedge$ (and), and $\neg$ (not). We denote by $\mathcal{B}(\mathbf{X})$ the set of propositional formulas over variables $\mathbf{X}$. A formula is positive if it contains only positive literals. A formula is in disjunctive normal form (DNF) if it is a disjunction of clauses. Given two disjoint sets of variables, $\mathbf{X}$ and $\mathbf{Y}$, a DNF formula is bipartite over $\mathbf{X}$ and $\mathbf{Y}$ if each clause has the form $x \wedge y$ with variables $x \in \mathbf{Y}$ and $y \in \mathbf{Y}$. The set of positive bipartite DNF formulas is denoted by 2DNF. A convenient way of representing a $2 \mathrm{DNF}$ formula is by labelling the variables by natural numbers, that is, $x_{1}, x_{2}, \ldots$ and $y_{1}, y_{2}, \ldots$, and representing each clause by a pair $(i, j) \in \mathbb{N} \times \mathbb{N}$. A set $E \subseteq \mathbb{N} \times \mathbb{N}$ of such pairs then defines the formula $\Psi=\bigvee_{(i, j) \in E} x_{i} y_{j}$

Semantics. Given the set $\mathbf{X}$ of variables, we denote by $\mathcal{I}$ the set of possible assignments of all variables from $\mathbf{X}$ to constants $\top$ and $\perp$. For a formula $\Psi$, its set of assignments is denoted by $\mathcal{I}(\Psi)=\{I: \operatorname{vars}(\Psi) \rightarrow\{\top, \perp\}\}$. A model of $\Psi$ is a satisfying assignment, that is, an assignment $I$ that maps $\Psi$ to $\top$, also denoted by $I \models \Psi$. The set of models of $\Psi$ is denoted by $\mathcal{M}(\Psi)$. Counting the number of models (determining the number $\# \Psi=|M(\Psi)|$ ) is already \#P-hard for 2DNF Provan and Ball 1983.

Probabilistic interpretation. Let now $\mathbf{X}$ be a set of independent random and variables. For each variable $x \in \mathbf{X}$, let $P_{x}$ be the probability of $x$ being true; we assume $P_{x}>0$ without loss of generality. The probability mass function $\operatorname{Pr}(I)=\left[\prod_{x \in \mathbf{X}}^{I(x)=\top} P_{x}\right] \cdot\left[\prod_{x \in \mathbf{X}}^{I(x)=\perp}\left(1-P_{x}\right)\right]$ for each assignment $I \in \mathcal{I}$ and the probability measure $\operatorname{Pr}(E)=\sum_{I \in E} \operatorname{Pr}(I)$ for all $E \subseteq \mathcal{I}$ define the probability space $\left(\mathcal{I}, 2^{\mathcal{I}}, \operatorname{Pr}\right)$ that we call the probability space induced by $\mathbf{X}$.

A formula $\Psi$ over random variables is itself a random variable $\Psi: \mathcal{I} \rightarrow\{\top, \perp\}$ over $\left(\mathcal{I}, 2^{\mathcal{I}}, \operatorname{Pr}\right)$ by letting $\Psi: I \mapsto I(\Psi)$ and with probability distribution defined as

$$
\operatorname{Pr}(\Psi=\top)=\sum_{I \in \mathcal{I}, I \models \Psi} \operatorname{Pr}(I) .
$$

We write $P_{\Psi}$ or $P(\Psi)$ for $\operatorname{Pr}(\Psi=\top)$ and $P_{\neg \Psi}$ or $P(\neg \Psi)$ for $\operatorname{Pr}(\Psi=\perp)=1-\operatorname{Pr}(\Psi=\top)$. If $P_{x}=1 / 2$ for each variable $x$, the model counting problem reduces to the probability computation problem: $P_{\Psi}=2^{-|\operatorname{vars}(\Psi)|} \# \Psi$, and the latter problem is \#P-hard for $2 \mathrm{DNF}$.

Binary decision diagrams $(B D D s)$. BDDs form a representation system for Boolean propositional formulas. A BDD over a set $\mathbf{X}$ of variables is a directed acyclic graph where inner nodes are labeled with variables from $\mathbf{X}$ and terminal nodes are $\top$ (true) and $\perp$ (false). Each inner node has two outgoing edges, for the case its variable is set to true (solid edge) and false (dotted edge) respectively. Each root-to-leaf path in a BDD is a (possibly partial) assignment of variables. A BDD is ordered (OBDD) if there is a total order $\Pi$ on its variables such that the variables visited by each path are in $\Pi$-order. A level in an OBDD corresponds to all nodes labeled with the same variable. The width 1 of a BDD is the maximum number of edges crossing the section of the OBDD between the nodes of any two consecutive levels, where edges incident to the same node are counted as one.

In this paper, we use of the following results on OBDDs:

LEMMA 2.1 (|WEGENER 2004]). Given an OBDD for a formula $\Psi$, the probability $P_{\Psi}$ can be computed in time linear in the size of the $O B D D$.

Let $\Phi_{1}, \Phi_{2}$ be two formulas, $\Pi$ be a fixed variable order on their variables, and $O_{1}$ and $O_{2}$

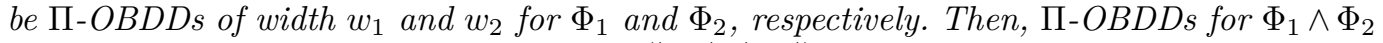
and $\Phi_{1} \vee \Phi_{2}$ can be constructed in time $O\left(\left|O_{1}\right| \cdot\left|O_{2}\right|\right)$ and have width at most $w_{1} \cdot w_{2}$.

\footnotetext{
${ }^{1} \mathrm{~A}$ different notion of BDD width refers to the maximum number of nodes in any level.
} 
Example 2.2. Figure 4 shows three OBDDs with the same variable order $\left(r_{1}, u_{1}, r_{2}, u_{2}, t_{1}\right.$, $\left.v_{1}, t_{2}, v_{2}\right)$. The path $r_{1} \stackrel{\mathrm{T}}{\rightarrow} \neg u_{1} \stackrel{\perp}{\rightarrow} r_{2} \stackrel{\perp}{\rightarrow} \perp$ encodes that under any truth assignment $\nu$ with $\nu\left(r_{1}\right)=\top$ and $\nu\left(\neg u_{1}\right)=\nu\left(r_{2}\right)=\perp$, the expression $\Psi_{1}=\left(r_{1} \neg u_{1} \vee r_{2} \neg u_{2}\right) \wedge\left(t_{1} \vee t_{2}\right)$ becomes false. The width of the left two OBDDs is three: There are three edges with different sinks crossing from level of $r_{2}$ to $\neg u_{2}$ and respectively from $t_{1}$ to $\neg v_{1}$.

The rightmost OBDD in Fig. 4 represents the disjunction of the two leftmost OBDDs and has width five. Intuitively, a disjunction of two OBBDs is computed in a top-down lockstep traversal of the input OBDDs using, e.g., the APPLY algorithm [Wegener 2004. For each node of a variable $x$ in both or one of the OBDDs, there is a node $x$ in the output OBDD with children computed recursively as the disjunctions of the OBDDs rooted at the children of the input OBDDs accessed by following the solid and respectively dotted edges. We choose the next node for the output OBDD following the common global variable order.

We can compute the probability of a BDD in one bottom-up pass. We exemplify for the OBDD of $\Psi_{1}$, where by $P(@ x)$ we denote the probability at node $x$ :

$$
\begin{aligned}
P\left(@ t_{2}\right) & =P_{t_{2}} \cdot P_{\top}+P_{\neg t_{2}} \cdot P_{\perp}=P_{t_{2}} \\
P\left(@ t_{1}\right) & =P_{t_{1}} \cdot P_{\top}+P_{\neg t_{1}} \cdot P\left(@ t_{2}\right)=P_{t_{1}}+\left(1-P_{t_{1}}\right) \cdot P\left(@ t_{2}\right) \\
P\left(@ \neg u_{2}\right) & =P_{\neg u_{2}} \cdot P\left(@ t_{1}\right)+\left(1-P_{\neg u_{2}}\right) \cdot P_{\perp}=\left(1-P_{u_{2}}\right) \cdot P\left(@ t_{1}\right) \\
P\left(@ r_{2}\right) & =P_{r_{2}} \cdot P\left(@ \neg u_{2}\right)+\left(1-P_{r_{2}}\right) \cdot P_{\perp}=P_{r_{2}} \cdot P\left(@ \neg u_{2}\right) \\
P\left(@ \neg u_{1}\right) & =P_{\neg u_{1}} \cdot P\left(@ t_{1}\right)+\left(1-P_{\neg u_{1}}\right) \cdot P\left(@ r_{2}\right) \\
P\left(@ r_{1}\right) & =P_{r_{1}} \cdot P\left(@ \neg u_{1}\right)+\left(1-P_{r_{1}}\right) \cdot P\left(@ r_{2}\right) .
\end{aligned}
$$

The probability of $\Psi_{1}$ is the probability of the OBDD, which is $P\left(@ r_{1}\right)$.

\subsection{Probabilistic databases}

Syntax and semantics. Probabilistic c-tables (pc-tables) are relational databases where each tuple is annotated with a formula over a set $\mathbf{X}$ of independent Boolean random variables Imielinski and Lipski 1984, Suciu et al. 2011. In its simplest form, each annotation formula is a distinct variable: This is the tuple-independent model considered in this article.

Under the possible worlds semantics, a pc-table $\mathcal{D}$ represents a finite set of possible worlds: Each total assignment $I$ of the variables in $\mathbf{X}$ defines a possible world representing a relational database consisting of exactly those tuples in $\mathcal{D}$ whose annotations are satisfied by $I$. The probability of each world is the product of probabilities of the variable assignments in $I$, that is, $\operatorname{Pr}(I)$ as defined above. This representation formalism is complete in the sense that it can represent arbitrary probability distributions over any finite set of possible worlds.

Query evaluation. Given a query $Q$ and a pc-table $\mathcal{D}$, the query evaluation problem is to compute the distinct tuples in the results of $Q$ in the worlds of $\mathcal{D}$ together with their probabilities. The probability $P(t \in Q(\mathcal{D}))$ of a tuple $t$ is the probability that $t$ is in the result of $Q$ in a world randomly drawn from $\mathcal{D}$. We adopt the intensional approach to query evaluation [Suciu et al. 2011]: For each result tuple $t$, first construct a propositional formula $\Phi_{t \in Q(\mathcal{D})}$ that annotates $t$ such that $P(t \in Q(\mathcal{D}))=P\left(\Phi_{t \in Q(\mathcal{D})}\right)$, then compute $P\left(\Phi_{t \in Q(\mathcal{D})}\right)$ as per Equation (1). The annotation of a Boolean query $Q$ is denoted by $\Phi_{Q(\mathcal{D})}$; when the context is clear, we often omit the explicit reference to the database $\mathcal{D}$ and simply write $\Phi_{Q}$. We next explain how to annotate the results of relational algebra queries on pc-tables.

The tuples together with their annotation in the result of a relational algebra query $Q$ can be computed directly from the input pc-table $\mathcal{D}$. This is achieved by rewriting $Q$ into a query $Q^{a}$ such that standard relational evaluation of $Q^{a}$ on $\mathcal{D}$ yields a pc-table representing the results of $Q$ in the worlds of $\mathcal{D}$. Algorithm 1 specifies such a rewriting function $\llbracket \cdot \rrbracket$. It assumes that the formulas annotating the tuples in the input pc-table are stored in a distinguished column called $\Phi$; for a relation $R$, we consider that this column 


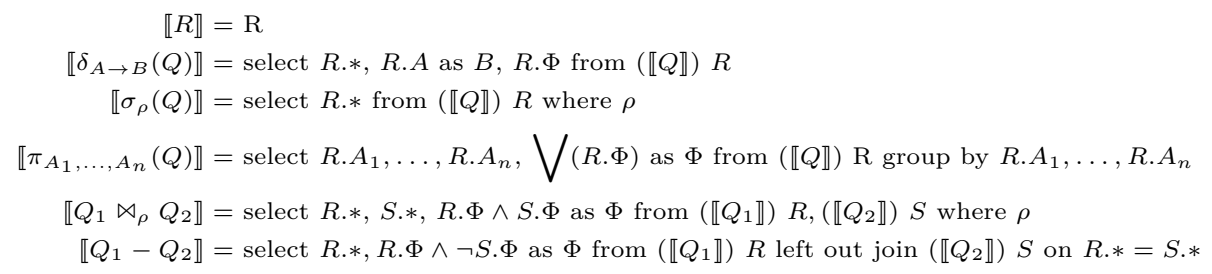

Algorithm 1: Computing annotated result tuples for RA queries on pc-tables.

is not selected by the selector R.*. The rewriting is expressed here in SQL and - besides a straightforward encoding of the relational operators in SQL - it constructs formulas annotating result tuples based on the formulas of input tuples as follows. In case of identity, selection, and renaming operators, the input annotations are just copied to the result. For projection, the formula of each distinct result tuple is constructed as the disjunction of all input tuples with the same restriction to the attributes in the projection list. For the join operator, the formula of a result tuple is the conjunction of the formulas of the contributing input tuples; to avoid cluttering, we slightly abuse notation in stating the attributes of the select clause: R.*, S.* means here the set-union of the attributes in $R$ and $S$. A tuple $t$ in the result of $Q_{1}-Q_{2}$ has annotation $\Phi_{1}$ if $t$ is in $Q_{1}$ with annotation $\Phi_{1}$ and $t$ is not in $Q_{2}$, and has annotation $\Phi_{1} \wedge \neg \Phi_{2}$ if $t$ is in $Q_{1}$ with annotation $\Phi_{1}$ and in $Q_{2}$ with annotation $\Phi_{2}$. These two cases are implemented in $\llbracket \cdot \rrbracket$ by a left outer join.

Example 2.3. Figure 2 shows pc-tables, where each tuple is annotated by a distinct Boolean random variable stored in column $\Phi$, and how annotations are propagated through the sub-queries of the depicted relational algebra query. The query result is the empty tuple annotated with the formula $\Phi=x_{1} y_{1} \vee x_{1} y_{2}$.

\section{HIERARCHICAL 1RA- QUERIES ARE TRACTABLE}

In this section we show the following result:

Lemma 3.1. Any hierarchical $1 R A^{-}$query $Q$ on tuple-independent databases has polynomial-time data complexity.

Proof. We assume without loss of generality that $Q$ is Boolean; if $Q$ is non-Boolean, we define a hierarchical Boolean $1 \mathrm{RA}^{-}$query for each tuple $t$ in the result of $Q$, where the tuple of attributes exported by $Q$ is set to $t$. We prove the lemma via a sequence of steps:

$Q$ is a hierarchical (Boolean) $1 \mathrm{RA}^{-}$query.

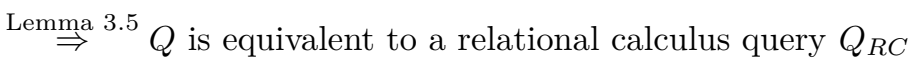

that is RC-hierarchical (Definition 3.2) and $\exists$-consistent (Definition 3.3).

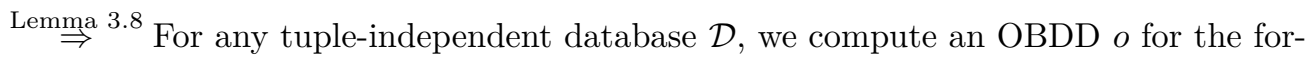
mula annotating the query result $Q_{R C}(\mathcal{D})$ in time and of size $\mathcal{O}\left(|\mathcal{D}| \cdot 2^{\left|Q_{R C}\right|}\right)$.

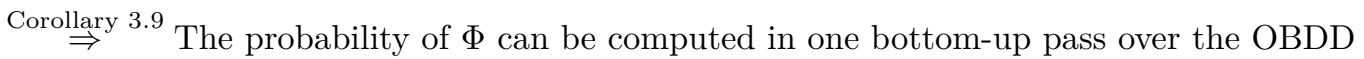
$o$, so in time $\mathcal{O}\left(|\mathcal{D}| \cdot 2^{\left|Q_{R C}\right|}\right)$.

The reason for translating $1 \mathrm{RA}^{-}$queries to relational calculus in the first step of the proof is that relational calculus is more flexible and allows to unfold negated expressions as per $\neg\left(Q_{1} \wedge Q_{2}\right) \equiv \neg Q_{1} \vee \neg Q_{2}$. The obtained rewritings are not arbitrary relational calculus queries. They are disjunctions of disjunction-free existential relational calculus queries that 
are expressible in the language $\mathrm{RC}^{\exists}$ and thus have no universal quantifiers. They are safe, that is, every query variable appears in at least one positive relation symbol. They use negation solely to capture the difference operator in relational algebra, which means that in an expression $Q_{1} \wedge \neg Q_{2}$, the results of $Q_{1}$ and $Q_{2}$ have the same arity. They are canonicalised in the sense that every occurrence of a relation symbol $R\left(Y_{1}, \ldots, Y_{m}\right)$ in a query rewriting has the same query variables $Y_{1}, \ldots, Y_{m}$. In contrast to the original $1 \mathrm{RA}^{-}$queries, the $\mathrm{RC}^{\exists}$ rewritings may have repeating relation symbols.

The $\mathrm{RC}^{\exists}$ rewritings enjoy two properties that are key to our query evaluation algorithm introduced later in this section. Firstly, they are hierarchical in a more syntactically restricted sense than the $1 \mathrm{RA}^{-}$queries.

Definition 3.2. An $\mathrm{RC}^{\exists}$ query $Q$ is RC-hierarchical if for every sub-query $\exists_{X}(F)$ in $Q$, the variable $X$ occurs in every relation symbol in $F$. We say that $X$ is root in $F$.

Secondly, $\mathrm{RC}^{\exists}$ rewritings allow for a total nesting order of its existential quantifiers for all their disjuncts.

Definition 3.3. A canonicalised $\mathrm{RC}^{\exists}$ query $Q$ is $\exists$-consistent if there exists a total order $>_{\exists}$ of the variable symbols in $Q$ such that $X>_{\exists} Y$ implies that there is no sub-query $\exists_{Y} F\left(\exists_{X}\right)$ in $Q$, where $F\left(\exists_{X}\right)$ denotes an expression that contains the quantifier $\exists_{X}$.

Intuitively, $\exists$-consistency for an $\mathrm{RC}^{\exists}$ query that is a conjunction or disjunction of subqueries means that these sub-queries have compatible join orders, that is, non-contradicting $>_{\exists}$ orders. This also means that their annotations, as well as the conjunction, disjunction, and negation of their annotations, can be compiled into OBDDs over the same variable order. In addition, the RC-hierarchical property effectively helps inferring from the order of the existential quantifiers an OBDD variable order under which the OBDD has size linear in the number of variables and thus in the database size, but possibly exponential in the query size. We next illustrate these concepts via an example.

Example 3.4. Consider the following three disjunction-free $\mathrm{RC}^{\exists}$ queries:

$$
\begin{aligned}
& Q_{1}=\exists_{A}(M(A) \wedge \neg R(A)) \wedge \exists_{B} N(B) \\
& Q_{2}=\exists_{A} M(A) \wedge \exists_{B}(N(B) \wedge \neg T(B)) \\
& Q_{3}=\exists_{A}(M(A) \wedge U(A)) \wedge \exists_{B}(N(B) \wedge V(B))
\end{aligned}
$$

All three queries are RC-hierarchical since for each occurrence of $\exists_{A}$ and $\exists_{B}, A$ and $B$, respectively, are root variables. Let us evaluate the queries over the database $\mathcal{D}$, viz:

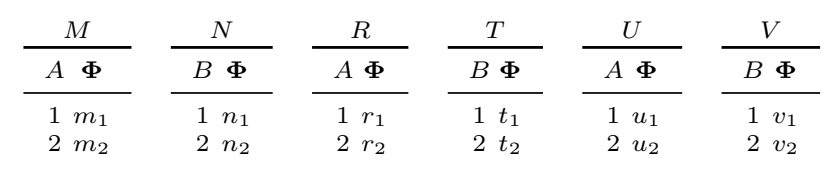

The annotations of the results for our queries evaluated on $\mathcal{D}$ are the read-once formulas

$$
\begin{aligned}
& \Phi_{1}=\left(m_{1} \bar{r}_{1} \vee m_{2} \bar{r}_{2}\right) \wedge\left(n_{1} \vee n_{2}\right) \\
& \Phi_{2}=\left(m_{1} \vee m_{2}\right) \wedge\left(n_{1} \bar{t}_{1} \vee n_{2} \bar{t}_{2}\right) \\
& \Phi_{3}=\left(m_{1} u_{1} \vee m_{2} u_{2}\right) \wedge\left(n_{1} v_{1} \vee n_{2} v_{2}\right)
\end{aligned}
$$




$$
\begin{aligned}
& \llbracket R \rrbracket=\{\{\operatorname{sch}(R)\} \mid R(\operatorname{sch}(R))\} \\
& \llbracket \pi_{-X}(Q) \rrbracket=\left\{H_{\llbracket Q \rrbracket} \backslash\{x\} \mid \mathrm{PUSH}^{\exists} X\left(F_{\llbracket Q \rrbracket}\right)\right\} \\
& \llbracket Q_{1} \bowtie_{\wedge_{i}\left(X_{i}=Y_{i}\right)} Q_{2} \rrbracket=\left\{H_{\llbracket Q_{1} \rrbracket} \cup H_{\llbracket Q_{2} \rrbracket} \mid \operatorname{ExPAND}\left(F_{\llbracket Q_{1} \rrbracket} \wedge F_{\llbracket Q_{2} \rrbracket}\left[\forall i: X_{i} / Y_{i} \rrbracket\right)\right\}\right. \\
& \left.\llbracket Q_{1}-\wedge_{i}\left(X_{i} \leftrightarrow Y_{i}\right) Q_{2} \rrbracket=\left\{H_{\llbracket Q_{1} \rrbracket} \mid \operatorname{EXPAND}\left(F_{\llbracket Q_{1} \rrbracket} \wedge \operatorname{PUSH}\right\urcorner\left(F_{\llbracket Q_{2} \rrbracket}\left[\forall i: X_{i} / Y_{i}\right]\right)\right)\right\}
\end{aligned}
$$

$\operatorname{PuSH}^{\exists} X($ Query $Q)$

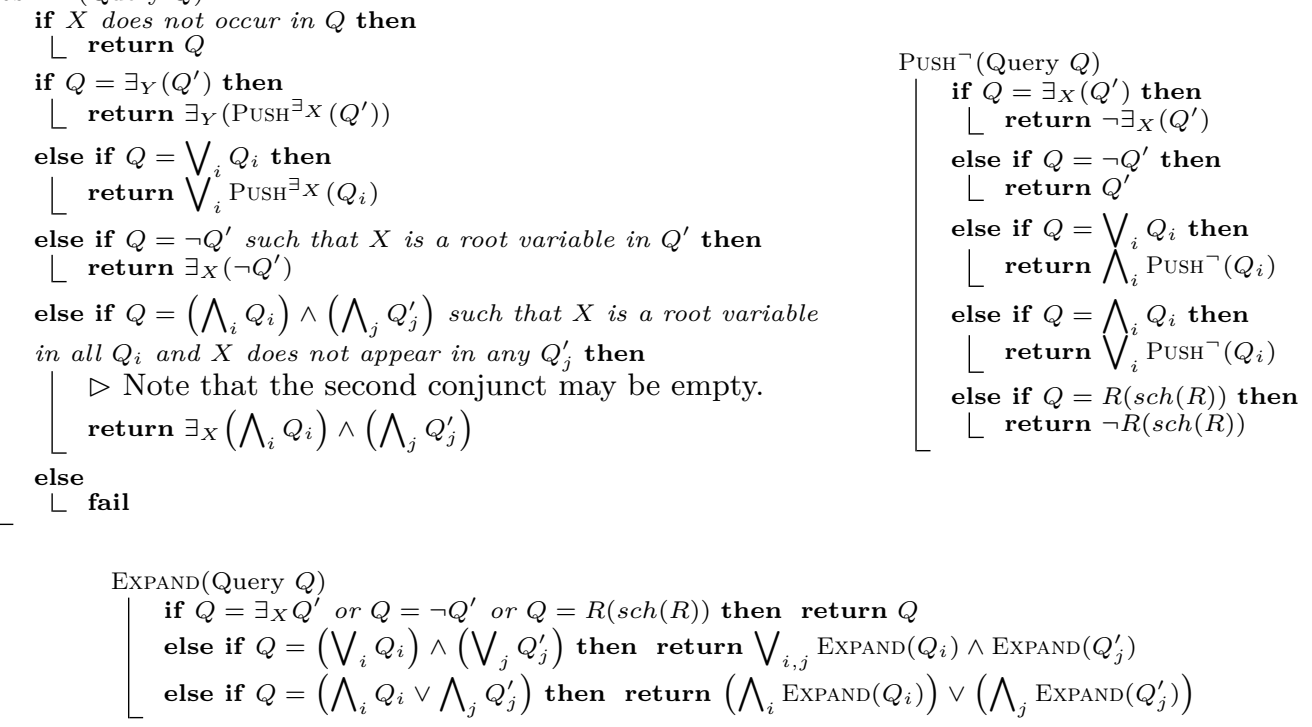

Algorithm 2: Translation function $\llbracket \cdot \rrbracket$ from $1 \mathrm{RA}^{-}$to $\mathrm{RC}^{\exists}$.

and, similarly to the first two OBDDs in Fig. 4, can be represented by OBDDs with one node per variable and width 2 under the following variable orders:

$$
\begin{aligned}
& \Pi_{1}: m_{1}, r_{1}, m_{2}, r_{2}, n_{1}, n_{2} \\
& \Pi_{2}: m_{1}, m_{2}, n_{1}, t_{1}, n_{2}, t_{2} \\
& \Pi_{3}: m_{1}, u_{1}, m_{2}, u_{2}, n_{1}, v_{1}, n_{2}, v_{2}
\end{aligned}
$$

Now consider the query $Q_{123}=Q_{1} \vee Q_{2} \vee Q_{3}$. As we show in Example 3.6 in the next section, this is obtained via translation of a hierarchical $1 \mathrm{RA}^{-}$query to relational calculus. It is RC-hierarchical and $\exists$-consistent. The variable orders $\Pi_{1}, \Pi_{2}$, and $\Pi_{3}$ are compatible in the sense that they can be extended into an order $\Pi_{123}$ over all variables:

$$
\Pi_{123}: m_{1}, r_{1}, u_{1}, m_{2}, r_{2}, u_{2}, n_{1}, t_{1}, v_{1}, n_{2}, t_{2}, v_{2}
$$

Following Lemma 2.1 the disjunction of the OBDDs of $\Phi_{1}, \Phi_{2}$, and $\Phi_{3}$ can be represented by an OBDD of width at most $2^{3}$ for the annotation $\Phi_{1} \vee \Phi_{2} \vee \Phi_{3}$ of query $Q_{123}$.

\subsection{From $1 \mathrm{RA}^{-}$to $\mathrm{RC}^{\exists}$}

Our evaluation algorithm for hierarchical $1 \mathrm{RA}^{-}$queries relies on a translation of $1 \mathrm{RA}^{-}$ queries into equivalent $\mathrm{RC}^{\exists}$ queries. The translation function $\llbracket \cdot \rrbracket$, which is given in Algorithm 2, is the standard recursive inside-out translation from relational algebra to safe relational calculus (Lemma 5.3.11, Abiteboul et al. 1995]), with the addition that after each recursive translation step we "flatten" the resulting $\mathrm{RC}^{\exists}$ query as follows: 
- Every $\exists$ quantifier is pushed as deep as possible in the $\mathrm{RC}^{\exists}$ query without pushing it past negation: $\exists_{X}$ distributes over disjunctions and is pushed past conjuncts in which $X$ does not appear. Lemma 3.5 shows that every $\exists_{X}$ quantifier can be pushed until $X$ becomes root, that is, $X$ occurs in all relation symbols in its scope.

— Every negation symbol is pushed as deep as possible in the $\mathrm{RC}^{\exists}$ query (as per $\neg(A \wedge B) \rightarrow$ $\neg A \vee \neg B$ and its dual) without pushing it past an existential quantifier.

- Conjunctions of disjunctions are eagerly expanded into disjunctions of conjunctions.

We may also apply the following two equivalence-preserving simplification rules, which are not necessary for the properties described in this article but useful for a practical implementation: Given $\mathrm{RC}^{\exists}$ expressions $Q_{1}$ and $Q_{2}$,

$$
Q_{1} \vee Q_{1} \wedge Q_{2} \rightarrow Q_{1} \text { and } \neg \exists_{X}\left(Q_{1}\right) \wedge \neg \exists_{X}\left(Q_{1} \wedge Q_{2}\right) \rightarrow \neg \exists_{X}\left(Q_{1}\right)
$$

The second rewriting is essentially a special case of the first rewriting, though our translation function can generate instances of left-hand sides of both rules.

Our translation has several desirable properties:

Lemma 3.5. For any hierarchical $1 R A^{-}$query $Q_{R A}$, the $R C^{\exists}$ rewriting $Q_{R C}=\llbracket Q_{R A} \rrbracket$ satisfies the following properties:

(a) $Q_{R C}$ is equivalent to $Q_{R A}$.

(b) $Q_{R C}$ is canonicalised.

(c) $Q_{R C}$ is a disjunction of disjunction-free $R C^{\exists}$ queries.

(d) For every variable $X$ occurring in $Q_{R C}, Q_{R C}$ has no sub-query of the form $\exists_{X}(Q) \wedge$ $F\left(\exists_{X}\right)$, where $F$ is an expression that contains the quantifier $\exists_{X}$.

(e) $Q_{R C}$ is RC-hierarchical.

(f) The quantifiers in $Q_{R C}$ can be ordered such that $Q_{R C}$ is $\exists$-consistent.

Proof. Property (a) holds since every rewriting step preserves equivalence.

Property (b). The attributes that are transitively joined in $Q_{R A}$ translate to the same variable name in $Q_{R C}$. This is indeed achieved in $\llbracket Q_{1} \bowtie Q_{2} \rrbracket$ and $\llbracket Q_{1}-Q_{2} \rrbracket$ by renaming all attributes in $Q_{2}$ to the corresponding variable name in $Q_{1}$. When a quantifier $\exists_{X}$ is introduced via $\llbracket \pi_{-X}(Q) \rrbracket$, then all occurrences of $X$ in $Q$ are in the scope of $\exists_{X}$; furthermore, the $\mathrm{PUSH}^{{ }}{ }$procedure never pushes $\exists_{X}$ past a relation symbol containing $X$ and hence every occurrence of $X$ is in the scope of a quantifier $\exists_{X}$.

Property (c). The combination of pushing down negation (PUSH $\urcorner$ ) and expanding conjunctions of disjunctions (EXPAND) is standard and yields an expression with disjunctions only at the top level.

Property (d). Let us assume that $Q_{R C}$ contains an expression $E=\exists_{X}(Q) \wedge F\left(\exists_{X}\right)$. Then the parse tree of $Q_{R C}$ contains a sub-tree of the following form:

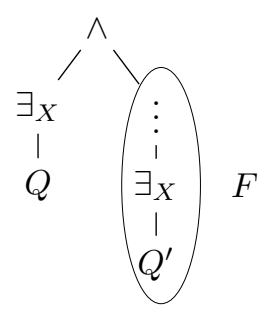

By construction using Algorithm 2, $E$ occurs positively in $Q_{R C}$, otherwise the algorithm would push the negation past the conjunction and yield $\neg \exists_{X}(Q) \vee \neg F$. 
Let us consider the possible transformation sequences that could have led to $E$. Since $Q_{R A}$ is non-repeating and since $Q_{R C}$ is canonicalised, the two $\exists_{X}$ quantifiers originate from one $\exists_{X}$ quantifier that has been applied to the entire sub-query as a result of the translation of $\llbracket \pi_{-X}(Q) \rrbracket$. The $\exists_{X}$ quantifier must subsequently have been "duplicated" as a result of distributing it through disjunctions in $\mathrm{PUSH}^{\exists}$. Since $\mathrm{PUSH}^{\exists}{ }^{\exists}$ does not distribute $\exists_{X}$ over a conjunction, the conjunction operator on top of this sub-query must have been flipped by a negation operator into a disjunction through which $\exists_{X}$ was distributed; in order to get back to a conjunction operator, there must have been an odd number of $\neg$ operators on top of the $\exists_{X}$ quantifier. This is a contradiction to the assumption that $Q_{R C}$ contains the expression $E$, where the $\exists_{X}$ quantifier appears positively.

Property (e). We show this property by induction on the different cases in the translation function $\llbracket \cdot \rrbracket$ in Algorithm 2 .

Base case: $Q_{R A}=R$. Since $\llbracket R \rrbracket=\{\{\operatorname{sch}(R)\} \mid R(\operatorname{sch}(R))\}$ does not contain existential quantifiers, $\llbracket R \rrbracket$ is vacuously RC-hierarchical.

Hypothesis: The $\mathrm{RC}^{\exists}$ expressions obtained by translating sub-queries of $Q_{R A}$ are RChierarchical.

Inductive step: Any step taken by the translation preserves the RC-hierarchical property.

The only translation step that may introduce a non-root $\exists_{X}$ quantifier is the case for $\llbracket \pi_{-X}\left(Q_{R A}\right) \rrbracket$. By the induction hypothesis, let $Q_{R C}=\llbracket Q_{R A} \rrbracket$ be the RC-hierarchical $\mathrm{RC}^{\exists}$ query resulting from the translation of $Q_{R A}$. Following properties (a) - (d), $Q_{R C}$ is canonicalised with respect to $Q_{R A}$, it is a disjunction of disjunction-free $\mathrm{RC}^{\exists}$ queries, and it does not contain an expression of the form $\exists_{X}(Q) \wedge F\left(\exists_{X}\right)$. We show that if $\operatorname{PusH}^{\exists_{X}}\left(Q_{R C}\right)$ fails, then $Q_{R A}$ is non-hierarchical. Conversely, for any hierarchical query $Q_{R A}, \mathrm{PuSH}^{\exists}$ always succeeds in pushing $\exists_{X}$ so that $X$ becomes a root variable in the scope of $\exists_{X}$.

The rule for $Q_{R C}=\bigvee_{j} Q_{j}$ pushes $\exists_{X}$ through the top-level disjunction and applies $\exists_{X}$ to each of the disjunction-free queries $Q_{j}$. Each $Q_{j}$ is a conjunction $\bigwedge_{i} q_{i}$ where each expression $q_{i}$ has the form $\exists_{Y}(q), \neg \exists_{Y}(q), R$, or $\neg R$. We analyse different cases separately:

(1) Variable $X$ occurs in exactly one expression $q_{i}$ :

(i) $q_{i}=\exists_{Y}(q)$. Then, by induction hypothesis, $Y$ is root in $q$ and this remains true after commuting $\exists_{X}$ and $\exists_{Y}: \exists_{Y}\left(\operatorname{PusH}^{\exists_{X}}(q)\right)$. We next analyse $\operatorname{PusH}^{\exists}(q)$.

(ii) $q_{i}=R$ or $q_{i}=\neg R$. Then, PUsH ${ }^{\exists_{X}}$ returns $\exists_{X} R$ or $\exists_{X} \neg R$, respectively, and $X$ is root in the scope of $\exists_{X}$ in both cases.

(iii) $q_{i}=\neg \exists_{Y}(q)$. If $X$ is root in $q$, then the rule for $Q_{R C}=\neg Q^{\prime}$ applies and we return $\exists_{X} \neg \exists_{Y}(q)$, in which $X$ is root. If $X$ were not root in $q$, then $q$ must contain two relations symbols such that by induction hypothesis $Y$ occurs in both relation symbols and $X$ occurs in one but not the other. Without loss of generality, let us assume that these relations are $T(Y)$ and $S(X, Y)$. Since $\llbracket \cdot \rrbracket$ does not commute $\neg$ and $\exists, Q_{R A}$ must contain a sub-query of the form $\pi_{-X}\left(Q_{1}-\pi_{-Y} Q_{2}\right.$, where $Q_{2}$ refers to relations $S$ and $T$ and $Q_{1}$ exports [X]; furthermore, since $Q_{R C}$ is canonicalised, no variable in $[Y]$ can occur in any relation symbol in $Q_{1}$. This means that $Q_{R A}$ contains three relation symbols $R^{[X][\neg Y]}, S^{[X][Y]}, T^{[\neg X][Y]}$ and is thus non-hierarchical.

(2) Variable $X$ occurs in more than one of the expressions $q_{i}$ :

(i) $X$ is root in all expressions $q_{i}$. Then, the case $Q_{R C}=\left(\bigwedge_{i} Q_{i}\right) \wedge\left(\bigwedge_{j} Q_{j}^{\prime}\right)$ applies in $\mathrm{PusH}^{\exists}$ and $X$ becomes root in the scope of $\exists_{X}$.

(ii) $X$ is not root in all expressions $q_{i}$. Then, $X$ occurs in one of them, say $q_{l}$, and there is a second expression, say $q_{k}$, that contains two relation symbols $S(X, Y)$ and $T(Y)$ such that $X$ occurs in one of them but not in the other. Thus $q_{k}$ has the form $\exists_{Y} Q(S, T)$ or $\neg \exists_{Y} Q(S, T)$ where by induction hypothesis $Y$ occurs in both $S$ 
and $T$. The latter case is equivalent to the above case (1iii). In the former case, if $q_{l}$ is a single relation $R$ (or its negation) in which $X$ occurs without a quantifier, then this relation cannot have variable $Y$ because all occurrences of $Y$ are in the scope of a quantifier, cf. Property (b). The relations $R, S, T$ thus render $Q_{R A}$ non-hierarchical. On the other hand, $q_{l}$ may have the form $\exists_{Z}(Q)$ where $Z$ is a variable different from $Y$ and $Q$ does not contain a quantifier $\exists_{Y}$, cf. Property (b). Then the relation in $q_{l}$ in which $X$ occurs, together with $S$ and $T$ render $Q_{R A}$ non-hierarchical.

Property (f). Assume $Q_{R C}$ contains two sub-queries $Q_{a}=\exists_{X} Q\left(\exists_{Y}\right)$ and $Q_{b}=\exists_{Y} Q\left(\exists_{X}\right)$ in which the order of the quantifiers $\exists_{X}$ and $\exists_{Y}$ is inconsistent. If one of the two sub-queries has the form $\exists_{X} \exists_{Y}\left(Q^{\prime}\right)$, then the order of the quantifiers may be switched to obtain $\exists$ consistent queries; conversely, there are two structurally different cases in which the order of the quantifiers cannot be switched: (i) $\exists_{X} Q\left(\neg \exists_{Y}\right)$, and (ii) $\exists_{X}\left[Q(X) \wedge Q^{\prime}\left(\exists_{Y}\right)\right]$. We consider the four combinations of these cases for the sub-queries $Q_{a}$ and $Q_{b}$ :

(1) They are of type (i): $Q_{a}=\exists_{X} Q_{1}\left(\neg \exists_{Y}\right)$ and $Q_{b}=\exists_{Y} Q_{2}\left(\neg \exists_{X}\right)$. Then, since $Q_{R C}$ is canonicalised and since the order of $\exists$ and $\neg$ is never swapped by the function $\llbracket \cdot \rrbracket$, the structure of $Q_{a}$ implies that $Q_{R C}$ has a sub-query of the form $\pi_{-X}\left(Q_{1}^{\prime}-\pi_{-Y}\left(Q_{1}^{\prime \prime}\right)\right)$ and the structure of $Q_{b}$ implies that $Q_{R C}$ has a sub-query of the form $\pi_{-Y}\left(Q_{2}^{\prime}-\pi_{-X}\left(Q_{2}^{\prime \prime}\right)\right)$. This is a contradiction.

(2) They are of type (ii): $Q_{a}=\exists_{X}\left[Q_{1}(X) \wedge Q_{1}^{\prime}\left(\exists_{Y}\right)\right]$ and $Q_{b}=\exists_{Y}\left[Q_{2}(Y) \wedge Q_{2}^{\prime}\left(\exists_{X}\right)\right]$. Then $Q_{1}$ contains a relation $R^{[X][\neg Y]}, Q_{2}$ contains a relation $T^{[\neg X][Y]}$, and, since $Q_{R C}$ and all its sub-queries are hierarchical, $Q_{1}^{\prime}$ contains a relation $S^{[X][Y]} . Q_{R A}$ is thus non-hierarchical since it contains the relations $R^{[X][\neg Y]}, S^{[X][Y]}, T^{[\neg X][Y]}$. This is a contradiction.

(3) $Q_{a}$ is of type (i) and $Q_{b}$ is of type (ii): $Q_{a}=\exists_{X} Q_{1}\left(\neg \exists_{Y}\right)$ and $Q_{b}=\exists_{Y}\left[Q_{2}(Y) \wedge Q_{2}^{\prime}\left(\exists_{X}\right)\right]$. Since there is no negation between $\exists_{Y}$ and $\exists_{X}$ in $Q_{b}$, there is also no difference operator between $\pi_{-X}$ and $\pi_{-Y}$ in $Q_{R A}$. Conversely, the negation between $\exists_{X}$ and $\exists_{Y}$ in $Q_{a}$ requires a difference operator between $\pi_{-X}$ and $\pi_{-Y}$ in $Q_{R A}$. This is a contradiction.

(4) $Q_{a}$ is of type (ii) and $Q_{b}$ is of type (i): This is symmetric to the previous case.

Property (d) disallows sub-queries of the form $\exists_{X}\left(Q_{1}\right) \wedge \exists_{X}\left(Q_{2}\right), \exists_{X}\left(Q_{1}\right) \wedge \neg \exists_{X}\left(Q_{2}\right)$, $\exists_{X}\left(Q_{1}\right) \wedge \neg \exists_{Y}\left(Q_{2} \wedge \neg \exists_{X}\left(Q_{3}\right)\right)$, but does not forbid $\neg \exists_{X}\left(Q_{1}\right) \wedge \neg \exists_{X}\left(Q_{2}\right)$ or $\exists_{X}\left(Q_{1}\right) \vee \exists_{X}\left(Q_{2}\right)$.

Example 3.6. Consider the following two $1 \mathrm{RA}^{-}$queries over the database schema $\left(M(A), N(B), R\left(A_{1}\right), T\left(B_{1}\right), U\left(A_{2}\right), V\left(B_{2}\right)\right)$ :

$$
\begin{aligned}
Q_{a} & =\pi_{\emptyset}\left[M \times N-A \leftrightarrow A_{1}, B \leftrightarrow B_{1}\right. \\
Q_{b} & \left.=\pi_{\emptyset}\left[R \times T-\pi_{A}(M \times N)-A_{1} \leftrightarrow A_{2}, B_{1} \leftrightarrow A_{1} \pi_{A_{1}} U \times V \times T-A_{1} \leftrightarrow A_{2}, B_{1} \leftrightarrow B_{2} U \times V\right]\right] .
\end{aligned}
$$

Query $Q_{a}$ translates to $Q_{123}$ from Example 3.4 where subsumed sub-queries are removed:

$$
\begin{aligned}
\llbracket Q_{a} \rrbracket= & \exists_{A} \exists_{B}(M(A) \wedge N(B) \wedge \neg[R(A) \wedge T(B) \wedge \neg(U(A) \wedge V(B))]) \\
= & \exists_{A} \exists_{B}(M(A) \wedge N(B) \wedge \neg[R(A) \wedge T(B) \wedge(\neg U(A) \vee \neg V(B))]) \\
= & \exists_{A} \exists_{B}(M(A) \wedge N(B) \wedge \neg[R(A) \wedge T(B) \wedge \neg U(A) \vee R(A) \wedge T(B) \wedge \neg V(B)]) \\
= & \exists_{A} \exists_{B}(M(A) \wedge N(B) \wedge(\neg R(A) \vee \neg T(B) \vee U(A)) \wedge(\neg R(A) \vee \neg T(B) \vee V(B))) \\
= & \exists_{A} \exists_{B}(M(A) \wedge N(B) \wedge(\neg R(A) \vee \neg T(B) \vee U(A) \wedge V(B))) \\
= & \exists_{A}(M(A) \wedge \neg R(A)) \wedge \exists_{B} N(B) \vee \exists_{A} M(A) \wedge \exists_{B}(N(B) \wedge \neg T(B)) \vee \\
& \exists_{A}(M(A) \wedge U(A)) \wedge \exists_{B}(N(B) \wedge V(B))=Q_{123} .
\end{aligned}
$$


Query $Q_{b}$ is similar to $Q_{a}$, but with additional projections on $A$ on both sides of the top-most difference operator (and hence $B$ is not in the equivalence class of $B_{1}$ and $B_{2}$ ):

$$
\begin{aligned}
\llbracket Q_{b} \rrbracket= & \exists_{A}\left[M(A) \wedge \exists_{B}(N(B)) \wedge \neg \exists_{B_{1}}\left(R(A) \wedge T\left(B_{1}\right) \wedge \neg\left(U(A) \wedge V\left(B_{1}\right)\right)\right)\right] \\
= & \left.\exists_{A}\left[M(A) \wedge \exists_{B}(N(B)) \wedge \neg \exists_{B_{1}}\left(R(A) \wedge T\left(B_{1}\right) \wedge \neg U(A) \vee R(A) \wedge T\left(B_{1}\right) \wedge \neg V\left(B_{1}\right)\right)\right)\right] \\
= & \exists_{A}\left[M(A) \wedge \exists_{B}(N(B)) \wedge\left(\neg R(A) \vee \neg \exists_{B_{1}}\left(T\left(B_{1}\right)\right) \vee U(A)\right)\right. \\
& \left.\wedge\left(\neg R(A) \vee \neg \exists_{B_{1}}\left(T\left(B_{1}\right) \wedge \neg V\left(B_{1}\right)\right)\right)\right] \\
= & \exists_{A}\left[M(A) \wedge \exists_{B}(N(B)) \wedge\left(\neg R(A) \vee \neg \exists_{B_{1}}\left(T\left(B_{1}\right)\right) \vee U(A) \wedge \neg \exists_{B_{1}}\left(T\left(B_{1}\right) \wedge \neg V\left(B_{1}\right)\right)\right)\right] \\
= & \exists_{A}(M(A) \wedge \neg R(A)) \wedge \exists_{B} N(B) \quad \vee \quad \exists_{A} M(A) \wedge \exists_{B} N(B) \wedge \neg \exists_{B_{1}} T\left(B_{1}\right) \quad \vee \\
& \exists_{A}(M(A) \wedge U(A)) \wedge \exists_{B} N(B) \wedge \neg \exists_{B_{1}}\left(T\left(B_{1}\right) \wedge \neg V\left(B_{1}\right)\right) .
\end{aligned}
$$

Both $\mathrm{RC}^{\exists}$ queries $\llbracket Q_{a} \rrbracket$ and $\llbracket Q_{b} \rrbracket$ satisfy Lemma 3.5 , for every quantifier $\exists_{A}\left(\exists_{B}\right.$ or $\left.\exists_{B_{1}}\right)$, $A\left(B\right.$ or $\left.B_{1}\right)$ is a root variable in its scope (Property (e)), and the nesting orders of these operators are consistent in all sub-queries (Property (f)).

The query translation can lead to large $\mathrm{RC}^{\exists}$ queries: A conservative upper bound on their sizes would be a non-elementary function of the size of the input $1 \mathrm{RA}^{-}$query, explained by the rapid increase in the size and number of disjuncts when pushing down negation, quantifiers, and conjunctions. A singly-exponential upper bound holds for $1 \mathrm{RA}^{-}$queries where for all projections $\pi_{-X}(Q)$ that are right descendants of a difference operator, attributes in the equivalence class $[X]$ occur in all relation symbols of $Q$ (that is, $X$ is root in $Q$ ). The query $Q_{a}$ in Example 3.6 satisfies this condition trivially, since it has no projection that is a right descendant of a difference operator. This conservative upper bound suffices for the data-complexity argument in Lemma 3.1 since the blowup is only in the query size. A practical implementation of Algorithm 2 would eagerly apply the simplification rules after each expansion step.

\subsection{OBDD Construction}

The penultimate step in the proof of Lemma 3.1 is the OBDD compilation of the annotation $\Phi$ of the $\mathrm{RC}^{\exists}$ query $Q_{R C}$, which is the rewriting of an input hierarchical $1 \mathrm{RA}^{-}$query $Q_{R A}$ as per Lemma 3.5. This OBDD has a total order $\Pi$ over the Boolean variables annotating the input tuples that can be derived from the structure of $Q_{R C}$. Let us first exemplify the construction of this order.

Example 3.7. The Boolean hierarchical $1 \mathrm{RA}^{-}$query $\pi_{\emptyset}\left[R \bowtie \pi_{X}(S-T)\right]$, over a probabilistic database with schema $(R(X), S(X, Y), T(X, Y))$, translates to the $\mathrm{RC}^{\exists}$ query

$$
Q_{R C}=\exists_{X}\left[R(X) \wedge \exists_{Y}(S(X, Y) \wedge \neg T(X, Y))\right] .
$$

Since $X$ is root in $Q_{R C}$, the OBDDs for $Q_{R C}$ 's annotations for different values of $X$ share no Boolean variables (that is, are independent) and can be concatenated. For each value $x$ in the active domain of $X$, we construct an OBDD for the annotation of the query $R(x) \wedge \exists_{Y}(S(x, Y) \wedge \neg T(x, Y))$; a good variable order for this OBDD is the sequence formed by the annotation of $R(x)$ and all annotations of $S(x, y)$ and $T(x, y)$ for all values $y$ in the active domain of $Y$. If we write $R\left(x_{i}\right)$ for the annotation of tuple $\left(x_{i}\right)$ in $R$, and similarly for $S$ and $T$, then the overall variable order is (e.g., for tuples with $X=x_{1}$ and $X=x_{2}$ ):

$$
\begin{aligned}
& R\left(x_{1}\right), S\left(x_{1}, y_{1}\right), T\left(x_{1}, y_{1}\right), S\left(x_{1}, y_{2}\right), T\left(x_{1}, y_{2}\right), S\left(x_{1}, y_{3}\right), T\left(x_{1}, y_{3}\right) \ldots \\
& R\left(x_{2}\right), S\left(x_{2}, y_{1}\right), T\left(x_{2}, y_{1}\right), S\left(x_{2}, y_{2}\right), T\left(x_{2}, y_{2}\right), S\left(x_{2}, y_{3}\right), T\left(x_{2}, y_{3}\right) \ldots
\end{aligned}
$$

The annotations can be concatenated in lexicographically ascending order of the values $x_{i}$ (any order of values $x_{i}$ for root variables leads to the same worst-case OBDD size): We first 
consider all annotations for $X=x_{1}$, then all annotations with $X=x_{2}$, and so on. For all annotations for $X=x_{1}$, we first consider those for $Y=y_{1}$, then those for $Y=y_{2}$, and so on. This variable order leads to a compact OBDD because the order of random variables annotating bindings of query variables $X, Y$ in the relations $R, S, T$ is compatible with the nesting order of the quantifiers $\exists_{X}$ and $\exists_{Y}$.

The RC $\mathrm{C}^{\exists}$ query $Q_{1}=\exists_{A}(R(A) \wedge \neg U(A)) \wedge \exists_{B} T(B)$ from Example 1.5 is the conjunction of two RC-hierarchical and $\exists$-consistent sub-queries: $Q_{A}=\exists_{A}(R(A) \wedge \neg U(A))$ and $Q_{B}=$ $\exists_{B} T(B)$. Similarly, $Q_{1}$ 's annotation $\Psi_{1}=\left(r_{1} \neg u_{1} \vee r_{2} \neg u_{2}\right) \wedge\left(t_{1} \vee t_{2}\right)$ is the conjunction of two formulas: $\varphi_{A}=r_{1} \neg u_{1} \vee r_{2} \neg u_{2}$ annotating $Q_{A}$ and $\varphi_{B}=t_{1} \vee t_{2}$ annotating $Q_{B}$. The query variables $A$ and $B$ are root in $Q_{A}$ and respectively $Q_{B}$. We independently construct OBDD variable orders for $\varphi_{A}$ and $\varphi_{B}$ and then concatenate them to obtain the overall variable order for the OBDD of $\Psi_{1}=\varphi_{A} \wedge \varphi_{B}$. Since $A$ is root, the OBDD variable order for $\varphi_{A}$ is a sequence of annotations $R\left(a_{1}\right), S\left(a_{1}\right), \ldots, R\left(a_{n}\right), S\left(a_{n}\right)$ for the domain $\left\{a_{1}, \ldots, a_{n}\right\}$ of $A$ : This is $r_{1}, u_{1}, r_{2}, u_{2}$. Similarly, we obtain the OBDD variable order $t_{1}, t_{2}$ for $\varphi_{B}$.

This variable order derived from the structure of the $\mathrm{RC}^{\exists}$ query rewritings leads to polysize OBDDs for query annotations. In general, however, finding an optimal OBDD variable order, that is, one that minimizes the size of the OBDD, is NP-complete Wegener 2004.

Lemma 3.8. For any $R C^{\exists}$ query $Q_{R C}$ that satisfies the properties of Lemma 3.5, the annotation $\Phi$ of $Q_{R C}$ on a tuple-independent database $\mathcal{D}$ can be represented by an $O B D D$ of size $\mathcal{O}\left(|\mathcal{D}| \cdot 2^{\left|Q_{R C}\right|}\right)$.

Proof. We prove the lemma for Boolean queries $Q_{R C}$; the non-Boolean case follows as per discussion in the proof of Lemma 3.1. Let the relation symbols in $Q_{R C}$ be $R_{1}, \ldots, R_{n}$, the query variables be $X_{1}, \ldots, X_{m}$, and let $\operatorname{ADom}\left(X_{i}\right)$ be the active domain of variable $X_{i}$. The annotation of tuple $t$ of relation $R_{i}$ is denoted by $R_{i}(t)$, e.g., the annotation of tuple $(a, b)$ in relation $R_{1}$ is $R_{1}(a, b)$. We assume without loss of generality that the order of the query variables $X_{1}, \ldots, X_{m}$ is such that $X_{i}>_{\exists} X_{j} \Leftrightarrow i<j$ with respect to the nesting order $>_{\exists}$ defined by the $\exists$-consistency of $Q_{R C}$; that is, $i<j$ allows for the quantifier nesting $\exists_{X_{i}} Q\left(\exists_{X_{j}}\right)$, but not $\exists_{X_{j}} Q\left(\exists_{X_{i}}\right)$. Since $Q_{R C}$ is canonicalised and $\exists$-consistent (Lemma 3.5), we can assume without loss of generality that the query variables in each relation symbol $R$ occur in $>_{\exists}$ order (we can always relabel the query and database schema such that the query variables occur in $>_{{ }}$-order $)$. For example, $Q_{R C}$ may contain $R\left(X_{1}, X_{5}, X_{7}\right)$, but not $R\left(X_{7}, X_{1}, X_{5}\right)$. Furthermore, we assume a total order over the active domain of the database such that for any $x_{i} \in \operatorname{ADom}\left(X_{i}\right)$ and $x_{j} \in \operatorname{ADom}\left(X_{j}\right)$ it holds that $x_{i}<x_{j} \Leftrightarrow i<j$; similarly for relation names: $R_{1}<R_{2}<\cdots<R_{n}$, where in addition the relation names are not part of the active domains of query variables and occur before the domain constants in this order.

We define a total order $\Pi$ on the annotations of the tuples in $\mathcal{D}$ as follows. We first associate with every annotation $R(t)$ the string string $(R(t))=t R$, e.g., annotation $R_{2}\left(A_{7}, B_{2}, C_{7}\right)$ is associated with the string $A_{7} B_{2} C_{7} R_{2}$. The order $\Pi$ is then defined as

$$
R(t)<_{\Pi} R^{\prime}\left(t^{\prime}\right) \Leftrightarrow \operatorname{string}(R(t))<_{\operatorname{lex}} \operatorname{string}\left(R\left(t^{\prime}\right)\right)
$$

where $<_{\text {lex }}$ is the lexicographic order on strings as defined by the total order of the active domain of the database and the relation names. The order $\Pi$ is uniquely defined by the order of the relation symbols and the order on the active domain of $\mathcal{D}$.

We show by structural induction over the annotation $\Phi$ that $\Phi$ has a $\Pi-O B D D$ of width $2^{\left|Q_{R C}\right|}$, where $\left|Q_{R C}\right|$ denotes the number of relation symbols in $Q_{R C}$ : 
- The base case is a Boolean variable $R(t)$ which corresponds to a trivial ח-OBDD with one variable $R(t)$ and width 2 (there are two edges between the level of the root node $R(t)$ and the next level of leaf nodes $\top$ and $\perp$ ).

- If $Q_{R C}=Q_{1} \wedge Q_{2}$ or $Q_{R C}=Q_{1} \vee Q_{2}$, then by induction hypothesis the annotations of $Q_{1}$ and $Q_{2}$ have $\Pi$-OBDDs of width $2^{\left|Q_{1}\right|}$ and $2^{\left|Q_{2}\right|}$, respectively. Then by Lemma 2.1 . the annotation of $Q_{R C}$ has a $\Pi$-OBDD of width $2^{\left|Q_{1}\right|} \cdot 2^{\left|Q_{2}\right|}=2^{\left|Q_{1}\right|+\left|Q_{2}\right|}=2^{\left|Q_{R C}\right|}$.

- If $Q_{R C}=\neg Q$, then by induction hypothesis $Q$ has a $\Pi$-OBDD of width $2^{|Q|}$. Swapping the leaf nodes $T$ and $\perp$ in this OBDD yields the required $\Pi$-OBDD for $Q_{R C}$.

- If $Q_{R C}=\exists_{X_{i}} Q$, then for every $x_{l} \in \operatorname{ADom}\left(X_{i}\right)$ the annotations $\Phi_{l}$ of queries $Q\left[x_{l} / X_{i}\right]$ are over disjoint sets of variables because $Q_{R C}$ is RC-hierarchical by Lemma 3.5 and $X_{i}$ is root in $Q$. Moreover, each annotation $\Phi_{l}$ has a $\Pi-O B D D$ of width $2^{|Q|}$ by induction hypothesis. Let $\operatorname{ADom}\left(X_{i}\right)=\left\{x_{1}, \ldots, x_{h}\right\}$ such that $x_{k}<_{\text {lex }} x_{l}$ if and only if $k<l$. The annotation $\Phi$ of $Q_{R C}$ is the disjunction $\bigvee_{x_{l} \in \operatorname{ADom}\left(X_{i}\right)} \Phi_{l}$. Since the formulas $\Phi_{l}$ are over disjoint sets of variables for distinct values of $l$, an OBDD for their disjunction is obtained by their concatenation in which the leaf node $\perp$ of the OBDD for $\Phi_{l}$ is replaced by the root node of the OBDD for $\Phi_{l+1}$.

It remains to show that this construction yields an OBDD over order П. Firstly, the OBDD for each annotation $\Phi_{l}$ is over order $\Pi$ by induction hypothesis; we show that for any two annotations $R\left(t_{k}\right)$ in $\Phi_{k}$ and $R^{\prime}\left(t_{l}\right)$ in $\Phi_{l}$ with $k<l$, it holds that $R^{\prime}\left(t_{k}\right)<_{\Pi} R^{\prime}\left(t_{l}\right)$; by the definition of $<_{\Pi}$, this is equivalent to showing $t_{k} R<_{\operatorname{lex}} t_{l} R^{\prime}$. The strings $t_{k}$ and $t_{l}$ are identical in the first $i-1$ places since, by construction, the occurrences of each variable $X_{j}$ with $j<i$ are set to the same constant. The lexicographic order of $t_{k}$ and $t_{l}$ - and hence the $\Pi$-order of $R\left(t_{k}\right)$ in $\Phi_{k}$ and of $R^{\prime}\left(t_{l}\right)$ in $\Phi_{l}$ - is determined by the values of $X_{i}$ in $t_{k}$ and in $t_{l}$; this value is $x_{l}$ in $t_{l}$ and $x_{k}$ in $t_{k}$. Since we concatenate the OBDDs in the order $\Phi_{1} \rightarrow \cdots \rightarrow \Phi_{h}$ and since $x_{1}<_{\text {lex }} \cdots<_{\text {lex }} x_{h}$, it follows that $t_{k}<_{\text {lex }} t_{l}$ and thus $R\left(t_{k}\right)<_{\Pi} R^{\prime}\left(t_{l}\right)$. The constructed OBDD has width $2^{\left|Q_{R C}\right|}=2^{|Q|}$, because the OBDD concatenation leaves the width unchanged.

The OBDD construction in the above proof shows that conjunction, disjunction, negation, and existential quantification of $\mathrm{RC}^{\exists}$ queries representing rewritings of hierarchical 1RA ${ }^{-}$ queries correspond to analogous operations on OBDDs representing the annotations of such queries. In particular, the width of the resulting OBDD is bounded above by the product of the widths of the input OBDDs. This is a conservative upper bound that allows a uniform and simple treatment of $\mathrm{RC}^{\exists}$ constructs in the proof. A tighter bound can be obtained via a more specific analysis: Any non-repeating RC-hierarchical $\mathrm{RC}^{\exists}$ query $Q$ admits an OBDD of width at most $|Q|$ and size linear in the input database size and independent of the query size Olteanu and Huang 2008. This tighter bound on the OBDD width can be immediately extended to $\exists$-consistent conjunction and disjunction of such queries $Q_{1}, \ldots, Q_{n}$ : The resulting OBDD has width $\left|Q_{1}\right| \cdot \ldots \cdot\left|Q_{n}\right|$, which is smaller than $2^{\left|Q_{1}\right|+\cdots+\left|Q_{n}\right|}$ as used in the proof.

We can now use both Lemmata 2.1 and 3.8 to obtain the polynomial-time computation of query probability, or equivalently of the formula annotating the query result:

Corollary 3.9 (Lemmata 2.1, 3.8). Let $Q_{R C}$ be a $R C^{\exists}$ query satisfying the properties of Lemma 3.5, $\mathcal{D}$ a tuple-independent database, and $\varphi$ the formula annotating the query result $Q_{R C}(\mathcal{D})$. Then, the probability of $\varphi$ can be computed in time $\mathcal{O}\left(|\mathcal{D}| \cdot 2^{\left|Q_{R C}\right|}\right)$.

\section{DATABASE CONSTRUCTION SCHEME USED IN HARDNESS REDUCTIONS}

We next present a database construction scheme that prescribes how to populate relations used in a non-hierarchical query such that the query result is annotated with a desired 2DNF formula. It focuses on two distinguished attribute classes $[A]$ and $[B]$ that witness the nonhierarchical property of the query. The construction scheme populates the attributes that 
are not in $[A]$ and $[B]$ such that the input values for the attributes in $[A]$ and $[B]$ along with their annotations are propagated through the query operators to the result. This scheme is used in Section 5 to construct hardness reductions for non-hierarchical queries.

We use two finite sets of constants, $\mathbf{K}_{A}$ and $\mathbf{K}_{B}$, and a constant $\square$ distinct from those in $\mathbf{K}_{A}$ and $\mathbf{K}_{B}$. In this section, the projection operator $\pi_{A}^{\Phi}$ retains both the attribute $A$ and the annotation column $\Phi$; in contrast, $\pi_{A}$ only selects the attribute $A$ without the annotation column $\Phi$. The notation $\left(a_{1}, \ldots, a_{n} \mid \Phi\left(a_{1}, \ldots, a_{n}\right)\right)$ denotes a tuple $\left(a_{1}, \ldots, a_{n}\right)$ annotated with formula $\Phi\left(a_{1}, \ldots, a_{n}\right)$.

\subsection{Preserving the data of one attribute}

We first consider the case of one distinguished attribute $A$. Let $\Phi$ be a total function on $\mathbf{K}_{A}$. A query $Q$ (and its particular case of a relation) is $A$-reducible to $\left(\mathbf{K}_{A}, \Phi\right)$ if the $[A]$ attributes of $Q$ are filled with all values from $\mathbf{K}_{A}$, all attributes not in $[A]$ are filled with $\square$, and the annotation of a tuple identified by $a \in \mathbf{K}_{A}$ is $\Phi(a)$ :

$$
\begin{array}{ll}
\pi_{A}^{\Phi}(Q)=\left\{(a \mid \Phi(a)) \mid a \in \mathbf{K}_{A}\right\} & \text { for any attribute } A \in[A] \\
\pi_{C}(Q)=\{(\square)\} & \text { for any attribute } C \notin[A]
\end{array}
$$

By $\operatorname{red}_{A}(Q)=\mathbf{K}_{A} \mid \Phi$ we denote that $Q$ is $A$-reducible to $\left(\mathbf{K}_{A}, \Phi\right)$. A query $Q$ that does not export $[A]$ is $\emptyset$-reducible to $(\square \mid \Phi)$, and we denote it by $\operatorname{red}_{\emptyset}(Q)=\square \mid \Phi$ where

$$
\begin{aligned}
& \pi_{\emptyset}^{\Phi}(Q)=\{(\Phi)\} \\
& \pi_{C}(Q)=\{(\square)\} \quad \text { for any attribute } C .
\end{aligned}
$$

We next define three classes of relations $\mathcal{Q}^{A}, \mathcal{Q}_{\text {fill }}$, and $\mathcal{Q}_{\emptyset}$ that are characterised by their $A$-reductions; let $\Phi_{\top}$ be the constant function $\Phi_{\top}()=.\top$.

$$
\begin{array}{rll}
Q^{[A]} \in \mathcal{Q}^{A} & \text { if } & \operatorname{red}_{A}(Q)=\mathbf{K}_{A} \mid \Phi \text { or } \operatorname{red}_{A}(Q)=\mathbf{K}_{A} \mid \neg \Phi \\
Q^{[A]} \in \mathcal{Q}_{\text {fill }} & \text { if } & \operatorname{red}_{A}(Q)=\mathbf{K}_{A} \mid \Phi_{\top} \\
Q^{[\neg A]} \in \mathcal{Q}_{\text {fill }} & \text { if } & \operatorname{red}_{\emptyset}(Q)=\square \mid \Phi_{\top} \\
Q \in \mathcal{Q}_{\emptyset} & \text { if } & Q=\emptyset
\end{array}
$$

Queries in $\mathcal{Q}^{A}$ are relations in which the values of $[A]$-attributes are populated with values from $\mathbf{K}_{A}$, and values for attributes not in $[A]$ are set to $\square$. There is a functional dependency $[A] \rightarrow \Phi$ such that every tuple is represented by its $A$-value $a$ and has a corresponding annotation $\Phi(a)$ or $\neg \Phi(a)$. Queries in $\mathcal{Q}_{\text {fill }}$ are like to $\mathcal{Q}^{A}$-queries with the difference that every tuple is annotated with $T$. Queries in $\mathcal{Q}_{\emptyset}$ are empty relations.

Example 4.1. Given the domain $\mathbf{K}_{A}=\left\{a_{1}, a_{2}, a_{3}\right\}$, the following relation $X$ over the distinguished attribute $A$ and two attributes $B, C$ with $B, C \notin[A]$ satisfies the properties of a $\mathcal{Q}^{A}$-query, and relation $Y$ is a $\mathcal{Q}_{\text {fill-query. }}$

\begin{tabular}{cccc}
\multicolumn{4}{c}{$\mathcal{Q}^{A}$-relation $X$} \\
\hline$A_{x}$ & $B_{x}$ & $C_{x}$ & $\mathbf{\Phi}$ \\
\hline$a_{1}$ & & & $x_{1}$ \\
$a_{2}$ & & & $x_{2}$ \\
$a_{3}$ & & & \\
\end{tabular}

\begin{tabular}{cccc}
\multicolumn{4}{c}{$\mathcal{Q}_{\text {fill }}$-relation $Y$} \\
\hline$A_{y}$ & $B_{y}$ & $C_{y}$ & $\mathbf{\Phi}$ \\
\hline$a_{1}$ & & & $\top$ \\
$a_{2}$ & & & $\top$ \\
$a_{3}$ & $\square$ & & $\top$
\end{tabular}

The functional dependency $A_{x} \rightarrow \Phi$ is trivially satisfied by $\Phi\left(a_{i}\right)=x_{i}$.

Figure 6 shows how $\mathcal{Q}^{A}, \mathcal{Q}_{\text {fill }}$, and $\mathcal{Q}_{\emptyset}$-queries are propagated through query operators: For query classes $\mathcal{Q}_{1}$ and $\mathcal{Q}_{2}$, the right-most column in the table shows the query class $\mathcal{Q}_{1} O p \mathcal{Q}_{2}$ for an operator $O p$ that is join or difference. 


\begin{tabular}{llll}
$\mathcal{Q}_{1}$ & $O p$ & $\mathcal{Q}_{2}$ & $\mathcal{Q}_{1} O p \mathcal{Q}_{2}$ \\
\hline $\mathcal{Q}^{A}$ & $\bowtie$ & $\mathcal{Q}_{\text {fill }}$ & $\mathcal{Q}^{A}$ \\
& - & $\mathcal{Q}_{\emptyset}$ & $\mathcal{Q}^{A}$ \\
$\mathcal{Q}^{A B}$ & $\bowtie$ & $\mathcal{Q}_{\text {fill }}$ & $\mathcal{Q}^{A B}$ \\
& - & $\mathcal{Q}_{\emptyset}$ & $\mathcal{Q}^{A B}$ \\
$\mathcal{Q}_{\text {fill }}$ & $\bowtie$ & $\mathcal{Q}^{A}$ & \\
& & $\mathcal{Q}^{A B}$ & $\mathcal{Q}^{A}$ \\
& & $\mathcal{Q}_{\text {fill }}$ & $\mathcal{Q}^{A B}$ \\
& - & $\mathcal{Q}^{A}$ & $\mathcal{Q}_{\text {fill }}$ \\
& & $\mathcal{Q}^{A B}$ & $\mathcal{Q}^{A}$ \\
& & $\mathcal{Q}_{\emptyset}$ & $\mathcal{Q}^{A B}$ \\
\hline
\end{tabular}

Fig. 6. Class membership of queries connecting classes $Q^{A}, Q_{\text {fill }}$, and $Q_{\emptyset}$ with operators $\bowtie,-$.

Example 4.2. Continuing Example 4.1, the join $X \bowtie Y$ on the corresponding $A, B, C$ attributes of $\mathcal{Q}^{A}$-query $X$ and $\mathcal{Q}_{\text {fill-query }} Y$ yields the following relation:

\begin{tabular}{ccccccc}
\multicolumn{7}{c}{$\mathcal{Q}^{A}$-query $X \bowtie Y$} \\
\hline$A_{x}$ & $A_{y}$ & $B_{x}$ & $B_{y}$ & $C_{x}$ & $C_{y}$ & $\mathbf{\Phi}$ \\
\hline$a_{1}$ & $a_{1}$ & & & - & & $x_{1}$ \\
$a_{2}$ & $a_{2}$ & & & & & $x_{2}$ \\
$a_{3}$ & $a_{3}$ & & & & & $x_{3}$
\end{tabular}

This join satisfies the conditions of a $\mathcal{Q}^{A}$-query as suggested by the rule $\mathcal{Q}^{A} \bowtie \mathcal{Q}_{\text {fill }} \rightarrow \mathcal{Q}^{A}$ in Fig. 6. Similarly, the difference $Y-X$ is also a $\mathcal{Q}^{A}$-query:

\begin{tabular}{lccl}
\multicolumn{4}{c}{$\mathcal{Q}^{A}$-query $Y-X$} \\
\hline$A_{y}$ & $B_{y}$ & $C_{y}$ & $\mathbf{\Phi}$ \\
\hline$x_{1}$ & & & $\neg \mathbf{x}_{1}$ \\
$x_{2}$ & & & $\neg \mathbf{x}_{2}$ \\
$x_{3}$ & & & $\neg \mathbf{x}_{3}$
\end{tabular}

For a query containing a $\mathcal{Q}^{A}$-relation $X^{[A]}$, we can populate its relations such that it becomes a $\mathcal{Q}^{A}$-query and thus satisfies Equation (2):

LEMMA 4.3. Given an attribute $A$, a relation $X$ exporting $A$, and a query $Q$ containing $X$ and exporting $A$. If $X \in \mathcal{Q}^{A}$, then the relations in $Q$ can be filled such that $Q \in \mathcal{Q}^{A}$.

Proof. Let $\mathcal{O P}$ - be the set of difference operators in $Q$ that do not have $X$ as a right descendant. We partition the relations of $Q$ into three sets:

$$
\begin{aligned}
\operatorname{rels}_{X} & =\{X\} \\
\operatorname{rels}_{\emptyset} & =\text { relations that are right descendants of a } \mathcal{O P}-\text { operator } \\
\operatorname{rels}_{\text {fill }} & =\text { all other relations }
\end{aligned}
$$

We populate every rels fill $_{\text {relation as a }} \mathcal{Q}_{\text {fill-query }}$ and every rels $\emptyset$ relation as a $\mathcal{Q}_{\emptyset \text {-query. }}$ The following inductive argument shows that every operator on the path in $Q$ between $X$ and the root of $Q$ is a $\mathcal{Q}^{A}$-query: First, this trivially holds at $X$ itself. Now let $O p$ be an operator on the path between $X$ and the root of $Q$. We have the cases:

$-Q_{L} \bowtie Q_{R}$, where without loss of generality $Q_{L}$ contains $X$. Then, $Q_{L}$ is a $\mathcal{Q}^{A}$-query, $Q_{R}$

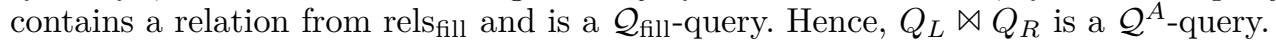

- $Q_{L}-Q_{R}$, where $Q_{L}$ contains $X$. Then the difference operator is in $\mathcal{O P}_{-}$and $Q_{R}$ is a $\mathcal{Q}_{\emptyset}$-query, $Q_{L}$ is a $\mathcal{Q}^{A}$-query. Hence, $Q_{L}-Q_{R}$ is a $\mathcal{Q}^{A}$-query.

$-Q_{L}-Q_{R}$, where $Q_{R}$ contains $X$. Then, $Q_{R}$ is a $\mathcal{Q}^{A}$-query, $Q_{L}$ contains a relation from rels $_{\text {fill }}$ and is a $\mathcal{Q}_{\text {fill-query. Hence, }} Q_{L}-Q_{R}$ is a $\mathcal{Q}^{A}$-query. 


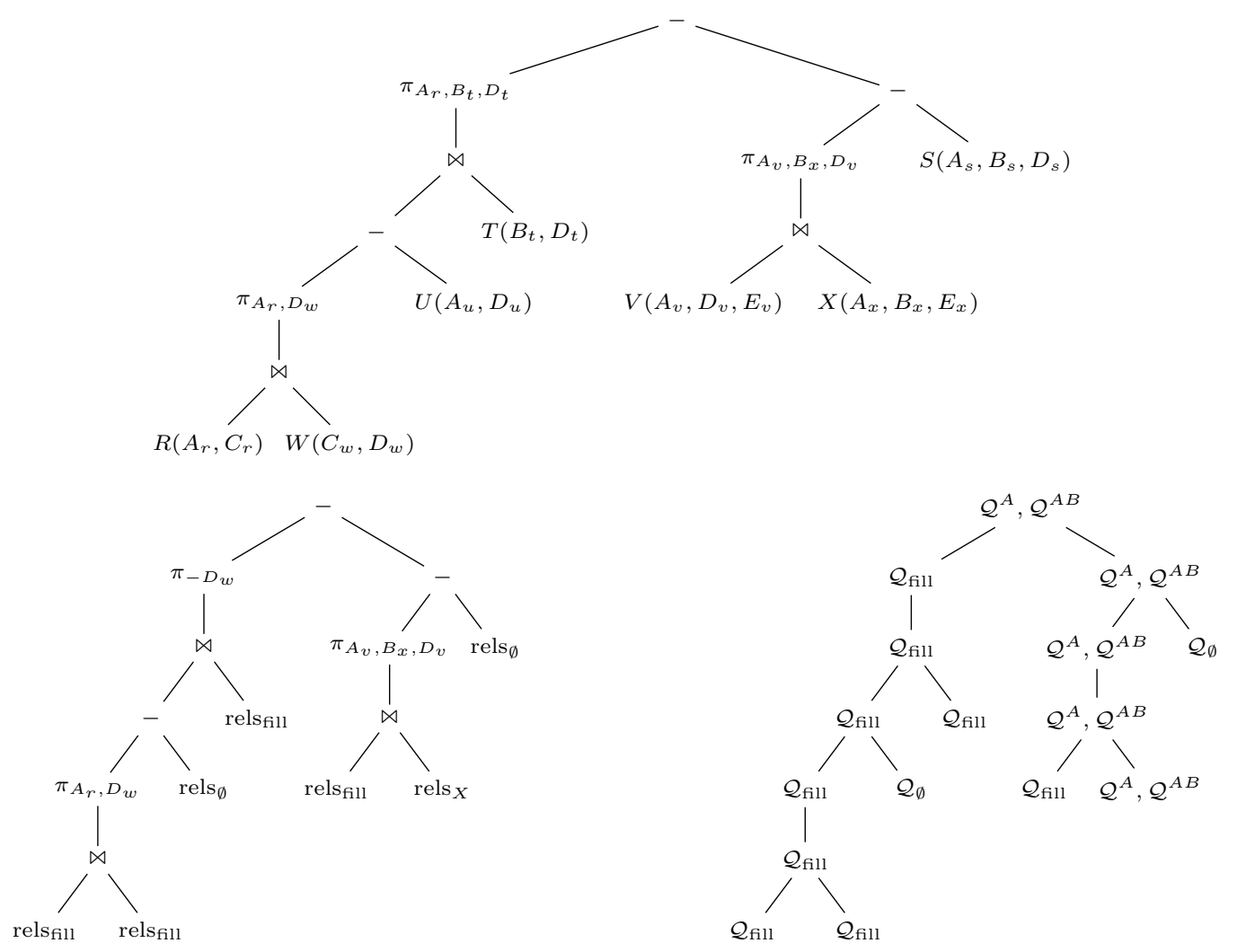

Fig. 7. A query $Q$ (top) and the corresponding partitioning of its relations into rels ${ }_{X}$, rels $\emptyset$, and rels fill $_{\text {fill }}$ relations (bottom-left), assuming that relation $X$ and its annotations are to be preserved by the filling. The bottom-right figure shows how the query classes of the sub-queries of $Q$ are propagated through query operators for two cases: (left) we preserve values and annotations for attributes in $[A]$, and then all subqueries rooted in operators on the path from $X$ to the root of $Q$ are $\mathcal{Q}^{A}$-queries; (right) we preserve for attributes in $[A]$ and $[B]$ and then these sub-queries become $\mathcal{Q}^{A B}$-queries. Since $X$ has odd polarity in $Q$, the annotations of tuples in $Q$ are the negated annotations of the corresponding tuples in $X$.

If $X$ has even polarity in $Q$, then the annotation $\Phi_{Q}(a)$ of a tuple $(a)$ in $\pi_{A}(Q)$ is the same as the corresponding annotation $\Phi_{X}(a)$ of a tuple $(a)$ in $\pi_{A}(X)$; if $X$ has odd polarity in $Q$, then $\Phi_{Q}(a)=\neg \Phi_{X}(a)$.

Example 4.4. Consider the query in Fig. 7 (top). We would like to preserve the attribute $A_{x}$ in relation $X$, where we use $\mathbf{K}_{A}=\left\{a_{1}, a_{2}, a_{3}\right\}$ as domain for $[A]$ and annotations $\Phi\left(a_{i}\right)=x_{i}$. The bottom-left graph shows the partition of $Q$ 's relations into rels $X=\{X\}$, rels $_{\text {fill }}=\{R, W, T, V\}$ and rels $\emptyset=\{U, S\}$. We set $U=S=\emptyset$, and for relations in rels fill we fill all attributes in $[A]=\left\{A_{r}, A_{u}, A_{v}\right\}$ with $\mathbf{K}_{A}$ and all other attributes with $\mathbf{\square}$. The bottom-right graph shows how the $\mathcal{Q}^{A}, \mathcal{Q}_{\text {fill }}$ and $\mathcal{Q}_{\emptyset}$ sub-queries are propagated through $Q$ 's operators. The children of the top difference operator are the following relations:

\begin{tabular}{|c|c|c|c|}
\hline \multicolumn{4}{|c|}{ Left sub-query of $\operatorname{root}(Q)$} \\
\hline$A_{r}$ & $B_{t}$ & $D_{t}$ & $\Phi$ \\
\hline$a_{1}$ & 口 & 口 & $\mathrm{T}$ \\
\hline$a_{2}$ & 뭄 & ㅁ & $T$ \\
\hline$a_{3}$ & [ & घ & $T$ \\
\hline
\end{tabular}

\begin{tabular}{llll}
\multicolumn{3}{c}{ Right } & sub-query of $\operatorname{root}(Q)$ \\
\hline$A_{v}$ & $B_{x}$ & $D_{v}$ & $\mathbf{\Phi}$ \\
\hline$a_{1}$ & & & $x_{1}$ \\
$a_{2}$ & & & $x_{2}$ \\
$a_{3}$ & & & $x_{3}$
\end{tabular}


Then, the relation represented by $Q$ is:

\begin{tabular}{llll}
\multicolumn{4}{c}{$Q$} \\
\hline$A_{r}$ & $B_{t}$ & $D_{t}$ & $\boldsymbol{\Phi}$ \\
\hline$a_{1}$ & & & $\neg x_{1}$ \\
$a_{2}$ & & & $\neg x_{2}$ \\
$a_{3}$ & $\square$ & & $\neg x_{3}$
\end{tabular}

$Q$ preserves $[A]$ and the annotations of relation $X$ : The annotations of tuples in $Q$ are the negation of the corresponding annotations in $X$ since $X$ has odd polarity in $Q$.

\subsection{Preserving the data of two attributes}

We can extend the previous construction scheme to the case of two attributes $A$ and $B$ from distinct classes. We first generalise the notation of $A$-reducible queries.

Let $\Phi^{A B}$ be a total function on $\mathbf{K}_{A} \times \mathbf{K}_{B}$, and let $\Phi^{A}$ be a total function on $\mathbf{K}_{A} \cup \mathbf{K}_{A} \times \mathbf{K}_{B}$ such that $\Phi^{A}(a)=\bigvee_{b \in \mathbf{K}_{B}} \Phi^{A}(a, b)$ for all $a \in \mathbf{K}_{A} ; \Phi_{\top}$ is the constant function $\Phi_{\top}()=.\top$. As before, a query (or relation) $Q$ is A-reducible to $\left(\mathbf{K}_{A}, \Phi^{A}\right)$, if

$$
\begin{aligned}
& \pi_{A}^{\Phi}(Q)=\left\{\left(a \mid \Phi^{A}(a)\right) \mid a \in \mathbf{K}_{A}\right\} \quad \text { for any attribute } A \in[A] \\
& \pi_{C}(Q)=\{(\square)\} \quad \text { for any attribute } C \notin[A]
\end{aligned}
$$

Similarly, $Q$ is $A B$-reducible to $\left(\mathbf{K}_{A} \times \mathbf{K}_{B}, \Phi^{A B}\right)$ if

$$
\begin{aligned}
\pi_{A B}^{\Phi}(Q) & =\left\{\left(a, b \mid \Phi^{A B}(a, b)\right) \mid a \in \mathbf{K}_{A}, b \in \mathbf{K}_{B}\right\} & & \text { for attributes } A \in[A], B \in[B] \\
\pi_{C}(Q) & =\{(\square)\} & & \text { for any attribute } C \notin[A] \cup[B]
\end{aligned}
$$

By $\operatorname{red}_{A B}(Q)=\mathbf{K}_{A} \times \mathbf{K}_{B} \mid \Phi^{A B}$ we denote that $Q$ is $A B$-reducible to $\left(\mathbf{K}_{A} \times \mathbf{K}_{B}, \Phi^{A B}\right)$. We next define the following classes of queries:

$$
\begin{aligned}
& Q^{[A][\neg B]} \in \mathcal{Q}^{A} \text { if } \operatorname{red}_{A}(Q)=\mathbf{K}_{A} \mid \Phi^{A} \text { or } \operatorname{red}_{A}(Q)=\mathbf{K}_{A} \mid \neg \Phi^{A} \\
& Q^{[A][B]} \in \mathcal{Q}^{A} \text { if } \operatorname{red}_{A B}(Q)=\mathbf{K}_{A} \times \mathbf{K}_{B} \mid \Phi^{A} \text { or } \operatorname{red}_{A B}(Q)=\mathbf{K}_{A} \times \mathbf{K}_{B} \mid \neg \Phi^{A} \\
& Q^{[A][B]} \in \mathcal{Q}^{A B} \text { if } \operatorname{red}_{A B}(Q)=\mathbf{K}_{A} \times \mathbf{K}_{B} \mid \Phi^{A B} \text { or } \operatorname{red}_{A B}(Q)=\mathbf{K}_{A} \times \mathbf{K}_{B} \mid \neg \Phi^{A B} \\
& Q^{[A][\neg B]} \in \mathcal{Q}_{\text {fill }} \text { if } \operatorname{red}_{A}(Q)=\mathbf{K}_{A} \mid \Phi_{\top} \\
& Q^{[A][B]} \in \mathcal{Q}_{\text {fill }} \text { if } \operatorname{red}_{A B}(Q)=\mathbf{K}_{A} \times \mathbf{K}_{B} \mid \Phi_{\top} \\
& Q^{[\neg A][\neg B]} \in \mathcal{Q}_{\text {fill }} \text { if } \operatorname{red}_{\emptyset}(Q)=\square \mid \Phi_{\top} \\
& Q \in \mathcal{Q}_{\emptyset} \text { if } Q=\emptyset
\end{aligned}
$$

Queries from these classes are propagated by query operators as depicted in Fig. 6 .

We now extend Lemma 4.3 to the case of one or two attributes:

Lemma 4.5. Given a query $Q$, attributes $A$ and $B$ from distinct classes in $Q$, and a relation $X$ in $Q$. If $X \in \mathcal{Q}^{A}$ and exports $A$ and not $B$, then the relations in $Q$ can be filled such that $Q \in \mathcal{Q}^{A}$ regardless whether $Q$ exports $A$ and not $B$, cf. Equation (6), or exports both $A$ and $B$, cf. Equation (7). If $X \in \mathcal{Q}^{A B}$ and $Q$ exports $A$ and $B$, then the relations in $Q$ can be filled such that $Q \in \mathcal{Q}^{A B}$, cf. Equation (8).

Proof. Let $\mathcal{O P}_{-}$be the set of operators that do not have $X$ as a right descendant. We partition the relations of $Q$ into three sets:

$$
\begin{aligned}
\operatorname{rels}_{X} & =\{X\} \\
\text { rels }_{\emptyset} & =\text { set of relations that are right descendants of an operator in } \mathcal{O P}_{-} \\
\text {rels }_{\text {fill }} & =\text { all other relations }
\end{aligned}
$$




\begin{tabular}{llll}
\multicolumn{4}{c}{$X$} \\
\hline$A_{x}$ & $B_{x}$ & $E_{x}$ & $\boldsymbol{\Phi}$ \\
\hline$a_{1}$ & $b_{1}$ & $\square$ & $x_{11}$ \\
$a_{1}$ & $b_{2}$ & $\square$ & $x_{12}$ \\
$a_{2}$ & $b_{1}$ & $\square$ & $x_{21}$ \\
$a_{2}$ & $b_{2}$ & $\square$ & $x_{22}$ \\
$a_{3}$ & $b_{1}$ & - & $x_{31}$ \\
$a_{3}$ & $b_{2}$ & $\square$ & $x_{32}$
\end{tabular}

\begin{tabular}{lll}
\multicolumn{3}{c}{$Q_{R W U}$} \\
\hline$A_{r}$ & $D_{w}$ & $\mathbf{\Phi}$ \\
\hline$a_{1}$ & $\square$ & $\top$ \\
$a_{2}$ & & $\top$ \\
$a_{3}$ & $\square$ & $\top$ \\
& &
\end{tabular}

\begin{tabular}{cccc}
\multicolumn{4}{c}{$Q_{R W U T}$} \\
\hline$A_{r}$ & $B_{t}$ & $D_{t}$ & $\mathbf{\Phi}$ \\
\hline$a_{1}$ & $b_{1}$ & $\square$ & $\top$ \\
$a_{1}$ & $b_{2}$ & & $\top$ \\
$a_{2}$ & $b_{1}$ & & $\top$ \\
$a_{2}$ & $b_{2}$ & & $\top$ \\
$a_{3}$ & $b_{1}$ & $\square$ & $\top$ \\
$a_{3}$ & $b_{2}$ & $\square$ & $\top$
\end{tabular}

\begin{tabular}{cccc}
\multicolumn{4}{c}{$Q_{V X S}$} \\
\hline$A_{v}$ & $B_{x}$ & $D_{v}$ & $\boldsymbol{\Phi}$ \\
\hline$a_{1}$ & $b_{1}$ & & $x_{11}$ \\
$a_{1}$ & $b_{2}$ & $\square$ & $x_{12}$ \\
$a_{2}$ & $b_{1}$ & & $x_{21}$ \\
$a_{2}$ & $b_{2}$ & & $x_{22}$ \\
$a_{3}$ & $b_{1}$ & & $x_{31}$ \\
$a_{3}$ & $b_{2}$ & & $x_{32}$
\end{tabular}

\begin{tabular}{cccc}
\multicolumn{4}{c}{$Q=Q_{R W U T}-Q_{V X S}$} \\
\hline$A_{r}$ & $B_{t}$ & $D_{t}$ & $\boldsymbol{\Phi}$ \\
\hline$a_{1}$ & $b_{1}$ & & $\neg x_{11}$ \\
$a_{1}$ & $b_{2}$ & & $\neg x_{12}$ \\
$a_{2}$ & $b_{1}$ & & $\neg x_{21}$ \\
$a_{2}$ & $b_{2}$ & $\neg x_{22}$ \\
$a_{3}$ & $b_{1}$ & $\neg x_{31}$ \\
$a_{3}$ & $b_{2}$ & & $\neg x_{32}$
\end{tabular}

Fig. 8. Relations used in Example 4.6

We first show $Q^{[A][B]} \in \mathcal{Q}^{A}$ given an $A$-reducible relation $X \in \mathcal{Q}^{A}$, cf. Equation (7). The relations in $Q$ are populated depending on their types. Every rels fill relation is populated

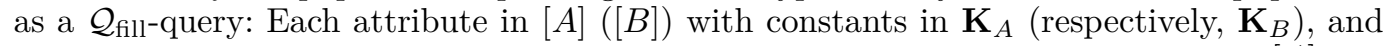
the other attributes with $\square$. Each relation in relsfill that exports attributes in both $[A]$ and $[B]$ is populated such that: its projection on any attribute in $[A]([B])$ is $\mathbf{K}_{A}$ (respectively, $\left.\mathbf{K}_{B}\right)$; its projection on any pair $(A, B)$ of attributes with $A \in[A]$ and $B \in[B]$ is $\mathbf{K}_{A} \times \mathbf{K}_{B}$; all attributes not in $[A]$ and $[B]$ take the value $\mathbf{D}$. Every rels $\emptyset$ relation is a $\mathcal{Q}_{\emptyset}$-query and thus kept empty.

We use a similar inductive argument as in the proof of Lemma 4.3 and show that every operator on the path in $Q$ between $X$ and the root of $Q$ is a $\mathcal{Q}^{A}$-query. Note however, that the lowest $\bowtie$-operator on the path from $X$ to the root of $Q$ that introduces a $[B]$ attribute marks the transition from a $\mathcal{Q}^{A}$ sub-query of type $Q^{[A][\neg B]}$ (Equation (6)) to $Q^{[A][B]}$ (Equation (7)). That is, the tuples $(a \mid \Phi(a))$ in $X$ are expanded to tuples $(a, b \mid \Phi(a))$ by means of the cross product between the $\mathcal{Q}^{A}$-relation that does not export $[B]$ and the $\mathcal{Q}_{\text {fill-relation that does export }[B] \text {. }}$

Finally, the case of a query $Q^{[A][B]}$ that contains a $\mathcal{Q}^{A B}$-relation $X^{[A][B]}$ follows as above and $Q \in \mathcal{Q}^{A B}$, that is, $Q$ is equivalent to $X$ with respect to attributes $[A]$ and $[B]$ and the annotations of the $(a, b)$-tuples.

The remark following Lemma 4.3 regarding the polarity and the sign of the annotations in $X$ and $Q$ carries over to our generalisation in Lemma 4.5

Example 4.6. Referring again to the query in Fig. 7. we would now like to preserve relation $X$ with respect to $[A]$ and $[B]$. We take the domains $\mathbf{K}_{A}=\left\{a_{1}, a_{2}, a_{3}\right\}$ and $\mathbf{K}_{B}=$ $\left\{b_{1}, b_{2}\right\}$ and the relation $X \in \mathcal{Q}^{A B}$ as depicted in Fig. 8 .

The sets of relations rels $X$, rels fill $_{\text {and }}$ and rels $\emptyset$ are identical to those in Example 4.4 and are depicted in the bottom-left graph in Fig. 7 The $\mathcal{Q}^{A B}$ and $\mathcal{Q}_{\text {fill }}$ classes through the query is depicted in the bottom-right graph. However, the relations represented by the different sub-queries may now be of different types.

Let us first consider the sub-query $Q_{R W U}$ consisting of relations $R, W, U$. Attribute $A_{r}$ is filled with $\mathbf{K}_{A}$, and attributes $C_{r}, C_{w}, D_{w}$ are set to $\mathbf{C}$; relation $U$ is set to $\emptyset$; all annotations

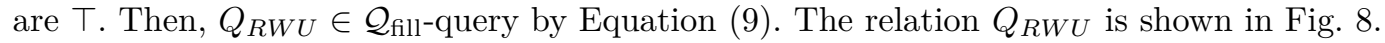

In relation $T$, attribute $B_{t}$ is filled with $\mathbf{K}_{B}$ and $D_{t}$ is filled with $\square$; its annotations are $\top$. The join between $Q_{R W U}$ and $T$ enforces an equality condition $D_{w}=D_{t}$ and yields the relation $Q_{R W U T}$ as depicted in Fig. $8 Q_{R W U T}$ is a $\mathcal{Q}_{\text {fill-query by virtue of Equation } 10 .}$. 
On the right side of the top most operator in $Q$, the sub-query $Q_{V X S}$ consisting of relations $V, X, S$ is in $\mathcal{Q}^{A B}$; it is depicted in Fig. 8. This leads to the $\mathcal{Q}^{A B}$-query $Q=$ $Q_{R W U T}-Q_{V X S}$ as depicted in the figure.

\section{NON-HIERARCHICAL 1RA- QUERIES ARE \#P-HARD}

In this section we show the following result:

LEMma 5.1. The data complexity of any non-hierarchical $1 R A^{-}$query is \#P-hard.

Proof. Given a $1 \mathrm{RA}^{-}$query $Q$ and any $2 \mathrm{DNF}$ formula $\Psi$, we use a reduction from the model-counting problem \# $\Psi$ by means of a construction of a database $\mathcal{D}$ such that $\Psi$ and the query result $Q(\mathcal{D})$ have the same probability. The reduction depends on structural properties of $Q$. We show that the non-hierarchical property is equivalent to matching a pattern (Definition 5.3 from the list of all possible patterns made up of inner nodes that are difference or join operators and leaves that correspond to three relations $R^{[A][\neg B]}, S^{[A][B]}$, and $T^{[B][\neg A]}$ for two distinct attribute classes $[A]$ and $[B]$. The notion of a match is then refined to that of an annotation-preserving match (Definition 5.7), for which a database construction scheme is possible such that the query result becomes annotated by $\Psi$.

The proof steps are summarised as follows:

$$
\begin{gathered}
Q \text { is non-hierarchical } \\
\Leftrightarrow \text { Proposition } 5.4 \\
Q \text { has a match with a pattern in Fig. } 9
\end{gathered}
$$

$$
\Leftrightarrow
$$

Lemma 5.8

$Q$ has an annotation-preserving match with a pattern in Fig. 9

$$
\stackrel{2}{\Rightarrow}
$$

$Q$ is \#P-hard.

\subsection{Patterns and matches}

We next define hard minimal query patterns and matches.

Definition 5.2. A pattern $P$ over attributes $A, B$ and relational operators $\mathrm{Op}_{1}, \mathrm{Op}_{2} \in$ $\{\bowtie,-\}$ is a binary tree with leaves $A, B$, and $A B$, root node $\mathrm{Op}_{1}$, and inner node $\mathrm{Op}_{2}$.

There are 48 different patterns: There are two distinct unlabeled binary trees with three leaves, the two operators can each be either join $(\bowtie)$ or difference $(-)$, and there are 6 possible orders of the labels $A, A B$, and $B$. Figure 9 shows 24 of the 48 patterns and omits for each pattern the symmetric pattern obtained by swapping leaves $A$ and $B$. By exploiting symmetries of the join operator in queries and patterns, it suffices to only consider 14 patterns (those shown in dark colour and not the source of directed arrows).

Definition 5.3. A $1 \mathrm{RA}^{-}$query $Q$ matches a pattern $P$ if there is mapping from the nodes of $P$ to nodes in the parse tree of $Q$ that preserves ancestor-descendant relationships: $A \mapsto R^{[A][\neg B]}, B \mapsto T^{[\neg A][B]}, A B \mapsto S^{[A][B]}, \mathrm{Op}_{1} \mapsto \mathrm{Op}_{1}$, and $\mathrm{Op}_{2} \mapsto \mathrm{Op}_{2}$. We also say that $Q$ is an $(R, S, T)$-match of $P$ to emphasise which relations establish the match.

Figures 1 and 10 show examples of queries matching patterns. Pattern matching is intimately linked to the non-hierarchical property:

Proposition 5.4. A $1 R A^{-}$query is non-hierarchical if and only if it matches one of the patterns in Fig. 9 .

Proof. $\Rightarrow$ : Let $Q$ be a non-hierarchical query. By Definition 1.2 there are two attributes $A$ and $B$ such that $Q$ contains relations $R^{[A][\neg B]}, S^{[A][B]}$, and $T^{[\neg A][B]} . Q$ matches exactly the pattern $P$ whose two operators correspond to the operators $O p_{1}$ and $O p_{2}$ in $Q$. The patterns 

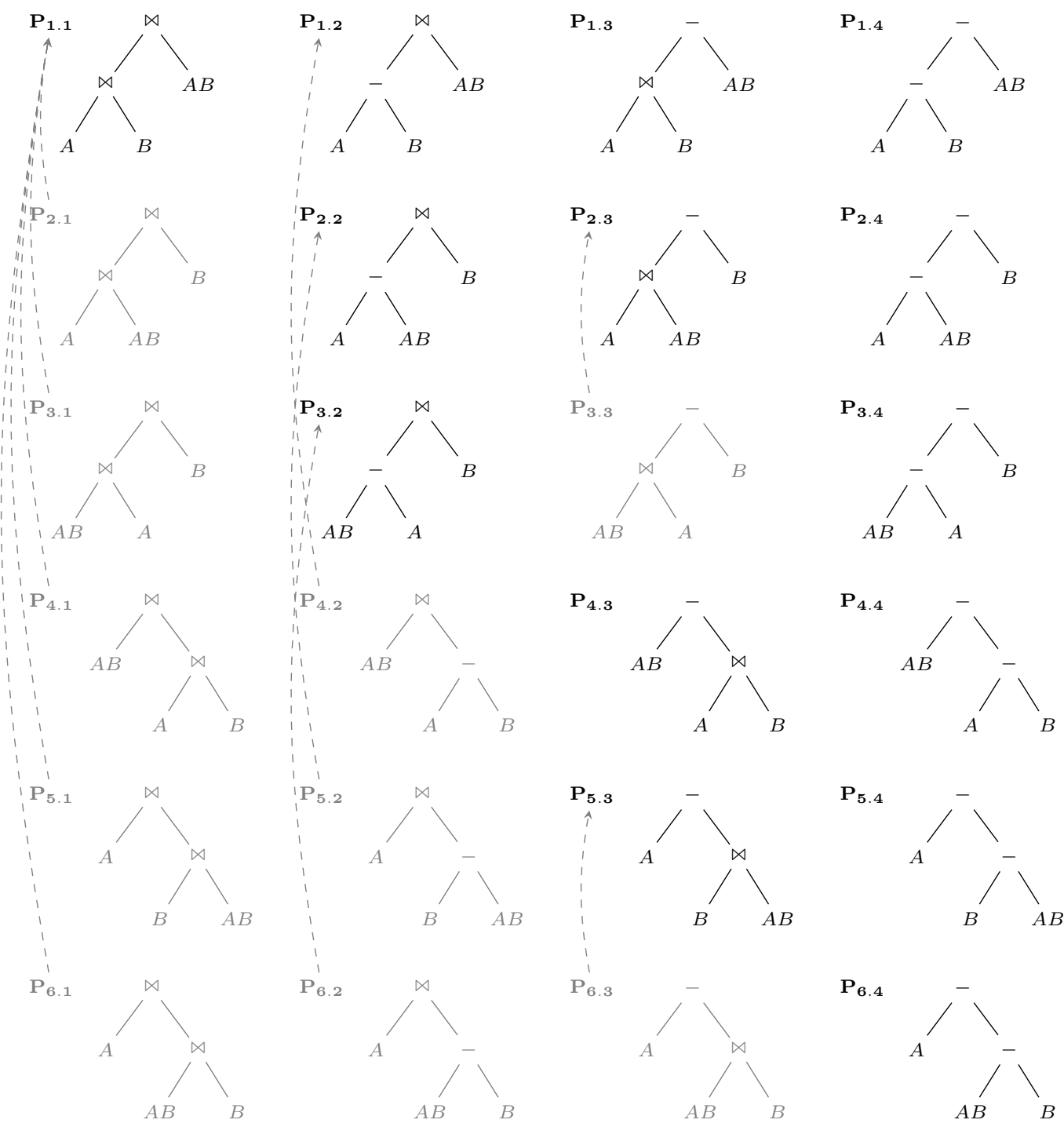

Fig. 9. The 24 query patterns $P_{1.1}, \ldots, P_{6.4}$. The 10 grey patterns can by reduced to other patterns as indicated by the arrows, since the labels $A$ and $B$ are symmetric and can be swapped, and the join $(\bowtie)$ operator is commutative and its sub-queries can also be swapped. Further 24 patterns can be obtained by swapping $A$ and $B$ in the above patterns.

are exhaustive in the sense that there is exactly one pattern per possible combination of the operators $\bowtie,-$.

$\Leftarrow$ : Every query $Q$ that matches a pattern contains three distinct relation symbols $R, S$, $T$ that render $Q$ non-hierarchical by Definition 1.2 .

The notion of a match is further specialised to that of an annotation-preserving match. Whereas the database construction scheme detailed in Section 4 does not work for general matches, it does work for annotation-preserving matches. We first define left-deep operators. 


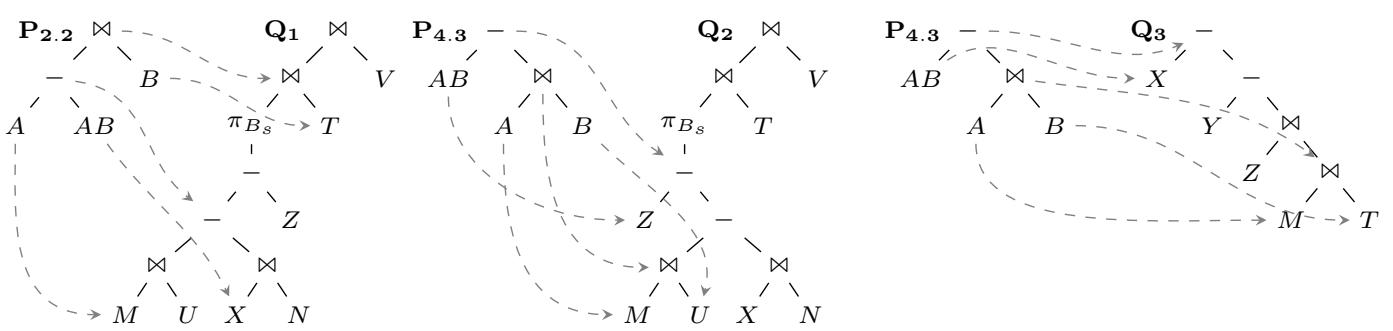

Fig. 10. Patterns $P_{2.2}$ and $P_{4.3}$ and parse trees of queries $Q_{1}, Q_{2}, Q_{3}$ over the schema $M\left(A_{m}\right), N\left(A_{n}\right)$, $T\left(B_{t}, C_{t}\right), U\left(B_{u}\right), V\left(B_{v}, C_{v}\right), X\left(A_{x}, B_{x}\right), Y\left(A_{y}, B_{y}\right), Z\left(A_{z}, B_{z}\right) . Q_{1}$ is an $(M, X, T)$-match of pattern $P_{2.2}$; it also matches other patterns and is an annotation-preserving $(M, X, T)$-match of $P_{2.2}$, since $O p_{2}$ (the least common ancestor of $M$ and $X$ ) is left-deep. Although $Q_{2}$ is an $(M, X, T)$-match of $P_{2.2}$, it is not an annotation-preserving match of $P_{2.2}$, since $O p_{2}$ is a right descendant of the top-most difference operator. However, $Q_{2}$ is an annotation-preserving $(M, Z, U)$-match of pattern $P_{4.3}$. Query $Q_{3}$ is an annotationpreserving $(M, X, T)$-match of pattern $P_{4.3}$.

Definition 5.5. An operator $O p$ is left-deep in a $1 \mathrm{RA}^{-}$query $Q$ if $O p$ is a left descendant of every difference operator on the path between the root of $Q$ and $O p$.

Example 5.6. In Fig. 10, the bottom-most difference operator in $Q_{1}$ is left-deep, while the bottom-most difference operator in $Q_{2}$ is not left-deep.

Definition 5.7. A $1 \mathrm{RA}^{-}$query $Q$ is an annotation-preserving $(R, S, T)$-match of a pattern $P$ over attributes $A$ and $B$ and operators $\mathrm{Op}_{1}$ and $\mathrm{Op}_{2}$ if: (1) $Q$ is an $(R, S, T)$-match of $P$; (2) For every difference operator $O p_{-}$in $Q$, if $O p_{1}$ is a right descendant of $O p_{-}$, then $O p_{-}$does not export [A] or [B]; (3) If $O p_{2}$ is a left descendant of $O p_{1}$ in $Q$, then $O p_{2}$ is left-deep in the sub-query rooted at $O p_{1}$. We say that $Q$ is an annotation-preserving $(R, S, T)$-match of $P$ to emphasise the relations establishing the match.

Figure 10 shows examples of annotation-preserving matches. We next look closer at the connection between matches and annotation-preserving matches. Lemma 5.8 establishes that any query that matches a pattern necessarily has an annotation-preserving match with a possibly different pattern. The relation symbols that establish the annotation-preserving match can be found by exploring the query tree in left-to-right depth-first in-order.

LEMma 5.8. Let $Q$ be a $1 R A^{-}$query and $o_{1}, \ldots, o_{n}$ be the sequence of its parse tree nodes in left-to-right depth-first in-order, and $Q_{1}, \ldots, Q_{n}$ be the corresponding sequence of sub-queries rooted at $o_{1}, \ldots, o_{n}$. If $Q_{i}$ is the first sub-query in the above order that matches a pattern in Fig. 9, then $Q_{i}$ is an annotation-preserving match of a pattern.

Proof. We show that $Q$ is an annotation-preserving match of a pattern $P^{\prime}$, that is, $Q$ satisfies the three properties from Definition 5.7 Let $O p_{1}$ and $O p_{2}$ be the two operators in $Q$ as established by a match with a pattern $P$.

Property 1 . This is already satisfied by definition: $Q_{i}$ already matches a pattern $P$. Without loss of generality, we assume that $Q_{i}$ is an $\left(R^{[A][\neg B]}, S^{[A][B]}, T^{[\neg A][B]}\right)$-match of $P$; the patterns are exhaustive in the sense that any arrangement of three relations $\left(R^{[A][\neg B]}, S^{[A][B]}, T^{[\neg A][B]}\right)$ and two operators corresponds to exactly one pattern. Note that $o_{i}=O p_{1}$ by construction.

Property 2. Proof by contradiction. Assume that there is an operator $o_{j}=-$ that is an ancestor of $o_{i}=O p_{1}$ such that $O p_{1}$ is a right descendant of $o_{j}$. It holds that $j<i$ due to the in-order of the query operators. Assume that $o_{j}$ exports $[A]$ or $[B]$. Then the left sub-query of $o_{j}$ contains at least one relation that exports $[A]$ or $[B]$. This relation together with a subset of $R, S, T$ would establish a match of $Q_{j}$ with some pattern; this is a contradiction 
to the assumption that $Q_{i}$ is the first sub-query (in the in-order sequence of sub-queries) to establish a match. Hence $o_{j}$ cannot export $[A]$ or $[B]$.

Property 3. Proof by contradiction. We are given that $O p_{2}$ is a left descendant of $O p_{1}$. Now assume that $O p_{2}$ is not left-deep in the left sub-query $Q_{L}$ of $O p_{1}$. Then, there is a top-most difference operator in $Q_{L}$, say $\mathrm{Op}_{-}$, such that $\mathrm{Op}_{2}$ is its right descendant. There are the following cases:

— Case 1: $R$ and $T$ are descendants of $O p_{2}$. Then $S$ is a right descendant of $O p_{1}$. Furthermore, $O p_{-}$exports $[A]$ and $[B]$ since every operator on the path between $R$ (T, respectively) must export $[A]\left([B]\right.$, respectively) in order for $O p_{1}$ to establish an equality or mapping for the attributes in $[A]$ and $[B]$ in $R, T$, and $S$. Thus the left sub-query of $O p_{-}$contains relations $X^{[A][\neg B]}$ and $Z^{[\neg A][B]}$, or it contains a relation $Y^{[A][B]}$. In the former case, the three relations $X, S, Z$ establish an annotation-preserving match, with $O p_{2}=O p_{-}$and $O p_{1}$ as before. The latter case is a contradiction to the assumption that $Q_{i}$ is the first sub-query in the in-order sequence of sub-queries of $Q$ that matches a pattern, because the sub-query rooted at $O p_{-}$precedes $Q_{i}$ in $Q$ 's in-order and matches a pattern via $R, Y, T$.

— Case 2: $R$ and $S$ are descendants of $O p_{2}$. Then $T$ is a right descendant of $O p_{1}$, and $O p_{-}$ exports $[B]$. Thus the left sub-query of $O p_{-}$contains a relation $Y^{[A][B]}$, or it contains a relation $X^{[\neg A][B]}$. In the former case, the three relations $R, Y, T$ establish a lineagepreserving match, with $O p_{2}=O p_{-}$and $O p_{1}$ as before. The latter case is a contradiction to the assumption that $Q_{i}$ is the first sub-query in the in-order sequence of sub-queries of $Q$ that matches a pattern, because the sub-query rooted at $O p_{-}$precedes $Q_{i}$ in $Q$ 's in-order and matches a pattern via $X, S, T$.

— Case 3: $T$ and $S$ are descendants of $O p_{2}$. Symmetric to case 2.

Example 5.9. Consider the query $Q_{2}$ in Fig. 10 The sub-query rooted at the top-most difference operator is the first one to match a pattern and also has an annotation-preserving ( $M, Z, U)$-match with $P_{4.3}$.

\subsection{Hardness reductions}

The 24 patterns in Fig. 9 are the smallest hard patterns for $1 \mathrm{RA}^{-}$, and any query that is an annotation-preserving match of one of them is hard for \#P.

LEMmA 5.10. The data complexity of any $1 R A^{-}$query that is an annotation-preserving match of one of the patterns in Fig. 9 is \#P-hard.

Putting together Proposition 5.4 and Lemmata 5.8 and 5.10, we obtain that the data complexity of all non-hierarchical $1 \mathrm{RA}^{-}$queries is \#P-hard.

The proof of Lemma 5.10 goes over each pattern case and shows hardness via a reduction from the \#2DNF problem; we only need to consider 14 distinct patterns, since, as shown in Fig. 9, ten patterns are equivalent to other ones.

Let $Q$ be a query that is an annotation-preserving $(R, S, T)$-match for a pattern $P$, and let $\Psi=\bigvee_{(i, j) \in E} x_{i} y_{j}$ be a $2 \mathrm{DNF}$ formula with $|E|$ clauses over disjoint variable sets $\mathbf{X}$ and $\mathbf{Y}$. We construct in polynomial time a tuple-independent database $\mathcal{D}$ using the database construction scheme in Section 4 such that the annotation of the query result $Q(\mathcal{D})$ is either $\Psi$ and hence $P_{Q(\mathcal{D})}=P_{\Psi}=\# \Psi \cdot 2^{-|\operatorname{vars}(\Psi)|}$, or $\neg \Psi$ and then $P_{Q(\mathcal{D})}=1-P_{\Psi}$.

In the following reductions, we use $\mathbf{K}(\mathbf{X})$ to denote the set of constants defined by the set $\mathbf{X}$ of Boolean variables; similar for $\mathbf{Y}$ and the union $\mathbf{X} \cup \mathbf{Y}$. While these constants are used for attributes in relations, their corresponding variables are used in propositional formulas for the special annotation column $\Phi$.

By Definition 5.7, the query $Q$ contains two distinct operators $O p_{1}$ and $O p_{2}$ and relations $R^{[A][\neg B]}, S^{[A][B]}, T=[\neg A][B]$. In the following, sub-queries $Q_{R}, Q_{S}, Q_{T}$ of $Q$ are defined to be the left or right sub-queries of $O p_{1}$ or $O p_{2}$ that contain exactly one of $R, S$, or $T$, 


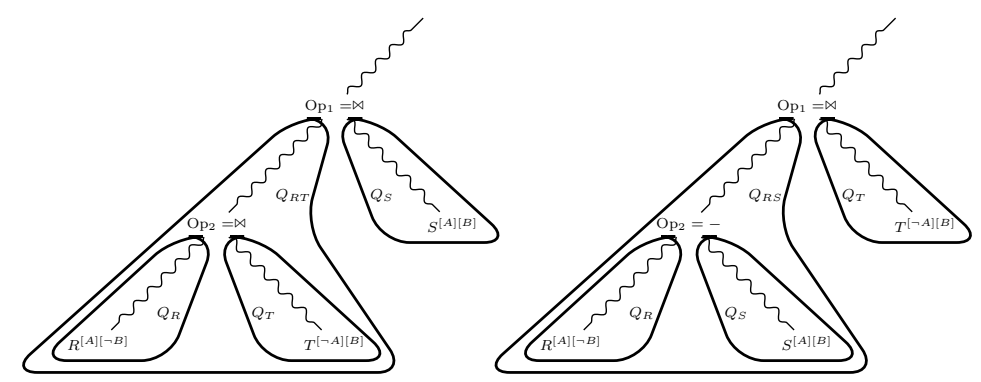

Fig. 11. Schematic illustrations of queries that are annotation-preserving matches for pattern $P_{1.1}$ (left) and $P_{2.2}$ (right). A curly edge indicates that further operators may occur on this path. By Definition 5.7 the operator $O p_{2}$ is left-deep in the left sub-query $Q_{R T}$, or $Q_{R S}$ respectively, of the operator $O p_{1}$, that is, it is the left descendant of any difference operator on the path between $O p_{1}$ and $O p_{2}$.

\begin{tabular}{ccc}
\multicolumn{3}{c}{$\mathcal{Q}^{A}$-relation $R$} \\
\hline$A_{r}$ & {$[\neg A]$} & $\mathbf{\Phi}$ \\
\hline $\mathrm{x}_{1}$ & & $\neg^{\mathrm{pol}} x_{1}$ \\
$\mathrm{x}_{2}$ & & $\neg^{\mathrm{pol}} x_{2}$ \\
$\cdots$ & & \\
$\mathrm{x}_{|\mathbf{X}|}$ & $\square$ & $\neg^{\mathrm{pol}} x_{\mid \mathbf{X}}$
\end{tabular}

\begin{tabular}{ccc}
\multicolumn{3}{c}{$\mathcal{Q}^{B}$-relation $T$} \\
\hline$B_{t}$ & {$[\neg B]$} & $\mathbf{\Phi}$ \\
\hline $\mathrm{y}_{1}$ & $\square$ & $\neg^{\mathrm{pol}} y_{1}$ \\
$\mathrm{y}_{2}$ & & $\neg^{\mathrm{pol}} y_{2}$ \\
$\cdots$ & & $\neg^{\mathrm{pol}} y_{|\mathbf{Y}|}$ \\
$\mathrm{y}_{|\mathbf{Y}|}$ & &
\end{tabular}

\begin{tabular}{cccc}
\multicolumn{4}{c}{$\mathcal{Q}^{A B}$-relation $S$} \\
\hline$A_{s}$ & $B_{s}$ & {$[\neg A],[\neg B]$} & $\mathbf{\Phi}$ \\
\hline $\mathrm{x}_{1}$ & $\mathrm{y}_{1}$ & & $\neg^{\mathrm{pol}} \top$ \\
$\mathrm{x}_{1}$ & $\mathrm{y}_{2}$ & & $\neg^{\mathrm{pol}} \top$ \\
$\mathrm{x}_{1}$ & $\mathrm{y}_{3}$ & & $\neg^{\mathrm{pol}} \perp$ \\
$\cdots$ & & $\neg^{\mathrm{pol}} \perp$
\end{tabular}

Fig. 12. Relations $R, S, T$ for the hardness reduction of a query with an annotation-preserving match for pattern $P_{1.1}$. The filling of $S$ assumes that the formula $\Psi$ is $x_{1} y_{1} \vee x_{1} y_{2}$ and thus tuples $\left(x_{1}, y_{1}\right)$ and $\left(x_{1}, y_{2}\right)$ are the only $S$-tuples annotated with $\top$ (assuming even polarity of $S$ in $Q_{S}$ ). To avoid clutter, the full polarity function is omitted from the annotation columns; for relation $R, \neg^{\text {pol }}$ stands for $\neg^{\text {pol }\left(Q_{R}, R\right)}$, for $T$ the polarity is $\neg^{\mathrm{pol}\left(Q_{T}, T\right)}$, and for $S$ it is $\neg^{\mathrm{pol}\left(Q_{S}, S\right)}$.

respectively, cf. Fig. 11. Additionally, if $O p_{2}$ has sub-queries $Q_{R}, Q_{S}$, then $Q_{R S}$ is the subquery of $O p_{1}$ that contains $Q_{R}$ and $Q_{S}$. Sub-queries $Q_{R T}$ and $Q_{S T}$ are defined similarly for matches in which $R$ and $T$ or $S$ and $T$ are descendants of $O p_{2}$, respectively.

The remainder of the proof treats the case of each pattern separately.

Amongst the patterns, $P_{1.1}$ is the only one needed to show hardness of non-hierarchical $1 \mathrm{RA}^{-}$queries without negation, that is, of non-repeating conjunctive queries studied in prior work Dalvi and Suciu 2007a]. Interestingly, the reduction for some patterns such as $P_{5.3}$ establishes that a query matching the pattern can be already hard for databases in which one relation is probabilistic and all other relations are certain.

\subsubsection{Reductions for patterns $P_{1.1}, P_{1.2}, P_{1.3}$, and $P_{1.4}$}

Pattern $P_{1.1}$. Let $Q$ be a query that is an annotation-preserving match of $P_{1.1}$. Figure 11 (left) depicts such a query $Q$, where $Q_{R}, Q_{S}, Q_{T}, Q_{R T}, Q_{R S T}$ denote sub-queries of $Q$. By Definition 5.7, $Q$ contains operators $O p_{1}=\bowtie$ and $O p_{2}=\bowtie$ and relations $R^{[A][\neg B]}, S^{[A][B]}, T^{[\neg A][B]}$. Every operator on the path $R-O p_{2}-O p_{1}-S$ exports $[A]$, and every operator on the path $T-O p_{2}-O p_{1}-S$ exports $[B]$. Moreover, the operator $O p_{1}$ expresses a join on both $[A]$ and $[B]$.

Since $Q$ is an annotation-preserving match, it satisfies the following additional structural properties: (1) If $O p_{1}$ is a right descendant of a difference operator, then this operator does not export $[A]$ or $[B]$. (2) Operator $O p_{2}$ is left-deep in $Q_{R T}$, that is, it is the left descendant of any difference operator on the path between $O p_{1}$ and $O p_{2}$.

We populate the relations $R, S, T$ as $\mathcal{Q}^{A}, \mathcal{Q}^{B}$, and $\mathcal{Q}^{A B}$-relations following Equations $(6)$ and (8). We thus fill the relation $R$ with constants from $\mathbf{K}(\mathbf{X})$ for $[A]$-attributes and annotations $\Phi_{R}$, the relation $T$ with constants from $\mathbf{K}(\mathbf{Y})$ for $[B]$-attributes and annotations $\Phi_{T}$, and the relation $S$ with the Cartesian product of the two sets of constants for attributes $[A]$ 
and $[B]$, and annotations $\Phi_{S}$. All other attributes are set to $\square$. The annotation functions are defined as follows:

$$
\begin{array}{lrl}
\Phi_{R}: \mathbf{K}(\mathbf{X}) \cup \mathbf{K}(\mathbf{X}) \times \mathbf{K}(\mathbf{Y}) \rightarrow \mathbf{X} \cup \mathbf{Y} & \Phi_{R}\left(\mathrm{x}_{\mathrm{i}}\right)=\Phi_{R}\left(\mathrm{x}_{\mathrm{i}}, \mathrm{y}_{\mathrm{j}}\right)=x_{i} \\
\Phi_{S}: \mathbf{K}(\mathbf{X}) \times \mathbf{K}(\mathbf{Y}) \rightarrow \mathbf{X} \cup \mathbf{Y} & \Phi_{S}\left(\mathrm{x}_{\mathrm{i}}, \mathrm{y}_{\mathrm{j}}\right)= \begin{cases}\top & \text { if }(i, j) \in E \\
\perp & \text { if }(i, j) \notin E\end{cases} \\
\Phi_{T}: \mathbf{K}(\mathbf{Y}) \cup \mathbf{K}(\mathbf{Y}) \times \mathbf{K}(\mathbf{X}) \rightarrow \mathbf{X} \cup \mathbf{Y} & \Phi_{T}\left(\mathrm{y}_{\mathrm{j}}\right)=\Phi_{T}\left(\mathrm{y}_{\mathrm{j}}, \mathrm{x}_{\mathrm{i}}\right)=y_{j}
\end{array}
$$

This database construction can be given more concisely using the notation from Section 4

$$
\begin{aligned}
\operatorname{red}_{A}(R) & =\mathbf{K}(\mathbf{X}) \mid \neg^{\operatorname{pol}\left(Q_{R}, R\right)} \Phi_{R} \\
\operatorname{red}_{A B}(S) & =\mathbf{K}(\mathbf{X}) \times \mathbf{K}(\mathbf{Y}) \mid \neg^{\mathrm{pol}\left(Q_{S}, S\right)} \Phi_{S} \\
\operatorname{red}_{B}(T) & =\mathbf{K}(\mathbf{Y}) \mid \neg^{\operatorname{pol}\left(Q_{T}, T\right)} \Phi_{T}
\end{aligned}
$$

Recall that the function $\operatorname{pol}(Q, R)$ defines the even (0) and odd (1) polarity of a relation symbol $R$ in the query $Q$. We use the convention $\neg^{1} \Phi \equiv \neg \Phi$ and $\neg^{0} \Phi \equiv \Phi$.

Figure 12 depicts the relations $R, S$, and $T$. By applying the results of Section 4 and Lemma 4.5, the remaining relations in $Q_{R}, Q_{S}$, and $Q_{T}$ can be filled such that $Q_{R} \in \mathcal{Q}^{A}$, $Q_{T} \in \mathcal{Q}^{B}$, and $Q_{S} \in \mathcal{Q}^{A B}$, that is, the values and annotations of $R, S$, and $T$ are preserved in $Q_{R}, Q_{S}$, and respectively $Q_{T}$. Since the filling of $R, S, T$ accounts for their polarity in their sub-queries $Q_{R}, Q_{S}, Q_{T}$, the latter relations take the following simple form ${ }^{2}$

$$
\operatorname{red}_{A}\left(Q_{R}\right)=\mathbf{K}(\mathbf{X})\left|\Phi_{R} \quad \operatorname{red}_{A B}\left(Q_{S}\right)=\mathbf{K}(\mathbf{X}) \times \mathbf{K}(\mathbf{Y})\right| \Phi_{S} \quad \operatorname{red}_{B}\left(Q_{T}\right)=\mathbf{K}(\mathbf{Y}) \mid \Phi_{T}
$$

Let us now define the following annotations:

$$
\begin{aligned}
\Phi_{R T}: \mathbf{K}(\mathbf{X}) \times \mathbf{K}(\mathbf{Y}) \rightarrow \mathbf{X} \cup \mathbf{Y} & \Phi_{R T}\left(\mathrm{x}_{\mathrm{i}}, \mathrm{y}_{\mathrm{j}}\right)=x_{i} y_{j} \\
\Phi_{R S T}: \mathbf{K}(\mathbf{X}) \times \mathbf{K}(\mathbf{Y}) \rightarrow \mathbf{X} \cup \mathbf{Y} & \Phi_{R S T}\left(\mathrm{x}_{\mathrm{i}}, \mathrm{y}_{\mathrm{j}}\right)= \begin{cases}x_{i} y_{j} & \text { if }(i, j) \in E \\
\perp & \text { if }(i, j) \notin E\end{cases}
\end{aligned}
$$

The sub-query $Q_{R} \bowtie Q_{T}$ is populated as follows:

$$
\operatorname{red}_{A B}\left(Q_{R} \bowtie Q_{T}\right)=\mathbf{K}(\mathbf{X}) \times \mathbf{K}(\mathbf{Y}) \mid \Phi_{R T} .
$$

By applying Lemma 4.5 to $Q_{R T}$, the annotations in the sub-query $Q_{R} \bowtie Q_{T}$ can be preserved by $Q_{R T}$. Since $O p_{2}$ is left-deep in $Q_{R T}, O p_{2}$ has even polarity in $Q_{R T}$ and hence the annotations of tuples in $Q_{R T}$ carry the same sign as in $Q_{R} \bowtie Q_{T}$. This yields

$$
\operatorname{red}_{A B}\left(Q_{R T}\right)=\mathbf{K}(\mathbf{X}) \times \mathbf{K}(\mathbf{Y})\left|\Phi_{R T} \quad \operatorname{red}_{A B}\left(Q_{R T} \bowtie Q_{S}\right)=\mathbf{K}(\mathbf{X}) \times \mathbf{K}(\mathbf{Y})\right| \Phi_{R S T} .
$$

The sub-query $Q_{R S T}=Q_{R T} \bowtie Q_{S}$ rooted at $O p_{1}$ thus has exactly one tuple $\left(x_{i}, y_{j}\right)$ annotated with $x_{i} y_{j}$ for each such clause in $\Psi$, and one tuple $\left(x_{i}, y_{j}\right)$ annotated with $\perp$ for each clause $x_{i} y_{j}$ not in $\Psi$.

Since by Definition 5.7 there does not exist a difference operator above $O p_{1}$ that exports $[A]$ or $[B]$, we can use the techniques of Lemma 4.5 to fill the relations representing sub-queries of $Q$ that are not descendants of $O p_{1}$ such that the annotations of $Q_{R S T}$ are preserved by any operator above $O p_{1}$. Finally, since by Definition $5.3 Q$ does not export $[A]$ or $[B]$, those attributes are eventually projected out above $O p_{1}$, yielding as result a single tuple annotated with the disjunction of the annotations of $Q_{R S T}$. If this projection is

\footnotetext{
${ }^{2}$ The match of $Q$ with pattern $P_{1.1}$ does not prohibit $O p_{2}$ from expressing a join on $[B]$. In that case, the sub-queries of $O p_{2}$ are $Q_{R}^{[A][B]}$ and $Q_{T}^{[A][B]}$ and satisfy $\operatorname{red}_{A B}\left(Q_{R}\right)=\mathbf{K}(\mathbf{X}) \times \mathbf{K}(\mathbf{Y}) \mid \Phi_{R}$ and $\operatorname{red}_{A B}\left(Q_{T}\right)=$ $\mathbf{K}(\mathbf{Y}) \times \mathbf{K}(\mathbf{X}) \mid \Phi_{T}$ in addition to the given reductions. Both the case of $O p_{2}$ carrying a join $[B]$ and the case of $O p_{2}$ not carrying this join are covered by the definition of $\mathcal{Q}^{A}$ in Equations [6] and [7].
} 
followed by difference operators (that do not export $[A]$ or $[B]$ ), then each of these difference operators will flip the sign of the annotation, that is, the annotation of $Q$ is $\neg^{p o l}\left(Q, O p_{1}\right) \Psi$. The probability $P_{Q(\mathcal{D})}$ of query $Q$ on the database constructed above is then $P_{\Psi}$ or $1-P_{\Psi}$.

Pattern $P_{1.2}$. A query that is an annotation-preserving match for $P_{1.2}$ has the same form as depicted in Fig. 11 (left), except that $O p_{2}=-$. The annotation functions $\Phi_{R}, \Phi_{S}, \Phi_{R T}$, and $\Phi_{R S T}$ are as in the case of pattern $P_{1.1}$, and the annotation function $\Phi_{T}$ carries an additional negation to account for the flipped polarity of $T$ (when compared to pattern $\left.P_{1.1}\right)$ due to the difference operator $O p_{2}$ :

$$
\Phi_{T}: \mathbf{K}(\mathbf{Y}) \cup \mathbf{K}(\mathbf{Y}) \times \mathbf{K}(\mathbf{X}) \rightarrow \mathbf{X} \cup \mathbf{Y} \quad \Phi_{T}\left(\mathrm{y}_{\mathrm{j}}\right)=\Phi_{T}\left(\mathrm{y}_{\mathrm{j}}, \mathrm{x}_{\mathrm{i}}\right)=\neg y_{j}
$$

We fill the relations $R, S$, and $T$ using $\operatorname{red}_{A}(R), \operatorname{red}_{A B}(S)$, and $\operatorname{red}_{B}(T)$ as for $P_{1.1}$.

The operator $O p_{2}$ exports $[A]$ and $[B]$ and thus the queries $Q_{R}$ and $Q_{T}$ export both $[A]$ and $[B]$. These queries represent the following relations:

$$
\begin{aligned}
& \operatorname{red}_{A}\left(Q_{R}\right)=\mathbf{K}(\mathbf{X}) \times \mathbf{K}(\mathbf{Y}) \mid \Phi_{R} \\
& \operatorname{red}_{B}\left(Q_{T}\right)=\mathbf{K}(\mathbf{Y}) \times \mathbf{K}(\mathbf{X}) \mid \Phi_{T}
\end{aligned}
$$

The sub-query $Q_{R}-Q_{T}$ is thus populated following $\operatorname{red}_{A B}\left(Q_{R}-Q_{T}\right)=\mathbf{K}(\mathbf{X}) \times$ $\mathbf{K}(\mathbf{Y}) \mid \Phi_{R T}$. The remainder of the reduction is identical to the case of pattern $P_{1.1}$.

Patterns $P_{1.3}$ and $P_{1.4}$. The reductions are identical to the cases of patterns $P_{1.1}$ and respectively $P_{1.2}$, except for the definition of $\Phi_{S}$ which carries an extra negation to account for the swapped polarity of $S$ :

$$
\Phi_{S}: \mathbf{K}(\mathbf{X}) \times \mathbf{K}(\mathbf{Y}) \rightarrow \mathbf{X} \cup \mathbf{Y} \quad \Phi_{S}\left(\mathrm{x}_{\mathrm{i}}, \mathrm{y}_{\mathrm{j}}\right)= \begin{cases}\perp & \text { if }(i, j) \in E \\ \top & \text { if }(i, j) \notin E\end{cases}
$$

5.2.2. Reduction for patterns $P_{2.2}, P_{2.3}$, and $P_{2.4}$. Let $Q$ be a query that is annotationpreserving match for one of these patterns. Such a query is depicted in Fig. 11 (right) for the case of $P_{2.2}$. The query $Q$ satisfies these structural constraints:

- Any operator on the path $R-O p_{2}-S$ exports [A], and every operator on the path $S$ $O p_{2}-O p_{1}-T$ exports $[B]$. The operator $O p_{2}$ expresses an equality condition on $[A]$, and the operator $O p_{1}$ expresses an join condition (for patterns $P_{2.1}$ and $P_{2.2}$ ) or a difference mapping (for patterns $P_{2.3}$ and $P_{2.4}$ ) on $[B]$.

- If $O p_{1}$ is a right descendant of a difference operator, then this operator does not export $[A]$ or $[B]$.

- The operator $\mathrm{Op}_{2}$ is left-deep in $Q_{R S}$, that is, it is the left descendant of any difference operator on the path between $O p_{1}$ and $O p_{2}$.

Pattern $P_{2.2}$. Relations $R, S, T$ are filled using $\Phi_{R}, \Phi_{S}$, and $\Phi_{T}$ exactly as in the case of pattern $P_{1.3}$. Additionally, define the following annotation functions:

$$
\begin{aligned}
\Phi_{R S}: \mathbf{K}(\mathbf{Y}) \cup \mathbf{K}(\mathbf{Y}) \times \mathbf{K}(\mathbf{X}) \rightarrow \mathbf{X} \cup \mathbf{Y} \quad \Phi_{R S}\left(\mathrm{y}_{\mathbf{j}}, \mathbf{x}_{\mathrm{i}}\right) & = \begin{cases}x_{i} & \text { if }(i, j) \in E \\
\perp & \text { if }(i, j) \notin E\end{cases} \\
\Phi_{R S}\left(\mathrm{y}_{\mathrm{j}}\right) & =\bigvee_{(i, j) \in E} x_{i} \\
\Phi_{R S T}: \mathbf{K}(\mathbf{Y}) \cup \mathbf{K}(\mathbf{Y}) \times \mathbf{K}(\mathbf{X}) \rightarrow \mathbf{X} \cup \mathbf{Y} \quad \Phi_{R S T}\left(\mathrm{y}_{\mathrm{j}}, \mathrm{x}_{\mathrm{i}}\right) & = \begin{cases}x_{i} y_{j} & \text { if }(i, j) \in E \\
\perp & \text { if }(i, j) \notin E\end{cases} \\
\Phi_{R S T}\left(\mathrm{y}_{\mathrm{j}}\right) & =y_{j} \wedge \bigvee_{(i, j) \in E} x_{i}
\end{aligned}
$$


Then $Q_{R}-Q_{S}$ and $Q_{R S}$ are $\mathcal{Q}^{B}$-queries that satisfy

$$
\operatorname{red}_{B}\left(Q_{R}-Q_{S}\right)=\mathbf{K}(\mathbf{Y})\left|\Phi_{R S} \quad \operatorname{red}_{B}\left(Q_{R S}\right)=\mathbf{K}(\mathbf{Y})\right| \Phi_{R S}
$$

and $Q_{T}$ is a $Q^{B}$-query with

$$
\operatorname{red}_{B}\left(Q_{T}\right)=\mathbf{K}(\mathbf{Y}) \mid \Phi_{T}
$$

Finally, the join $Q_{R S} \bowtie Q_{T}$ satisfies

$$
\operatorname{red}_{B}\left(Q_{R S} \bowtie Q_{T}\right)=\mathbf{K}(\mathbf{Y}) \mid \Phi_{R S T}
$$

and the reasoning about operators above $O p_{1}$ is as in the case of pattern $P_{1.1}$. The eventual projection $\pi_{-[B]}$ yields the nullary relation with one tuple annotated with $\neg^{\text {pol }\left(Q, O p_{1}\right)} \Psi$.

Pattern $P_{2.3}$. We fill the relations $R, S$, and $T$ as in the case of pattern $P_{1.2}$. The analysis is identical to $P_{2.2}$.

Pattern $P_{2.4}$. This case is identical to $P_{2.2}$, where the relation $T$ annotation uses the following function:

$$
\Phi_{T}: \mathbf{K}(\mathbf{Y}) \cup \mathbf{K}(\mathbf{Y}) \times \mathbf{K}(\mathbf{X}) \rightarrow \mathbf{X} \cup \mathbf{Y} \quad \Phi_{T}\left(\mathrm{y}_{\mathrm{j}}\right)=\Phi_{T}\left(\mathrm{y}_{\mathrm{j}}, \mathrm{x}_{\mathrm{i}}\right)=\neg y_{j}
$$

5.2.3. Reduction for patterns $P_{3.2}$ and $P_{3.4}$. The structure of queries matching any of these patterns is equivalent to those matching a pattern $P_{2 . x}$ with relations $R$ and $S$ swapped. The structural constraints remain the same and hence the reductions are very similar.

Pattern $P_{3.2}$. This case is similar to $P_{2.2}$ where $\Phi_{R}$ and $\Phi_{S}$ are negated:

$$
\begin{array}{lrl}
\Phi_{R}: \mathbf{K}(\mathbf{X}) \cup \mathbf{K}(\mathbf{X}) \times \mathbf{K}(\mathbf{Y}) \rightarrow \mathbf{X} \cup \mathbf{Y} & \Phi_{R}\left(\mathrm{x}_{\mathrm{i}}\right)=\Phi_{R}\left(\mathrm{x}_{\mathrm{i}}, \mathrm{y}_{\mathrm{j}}\right)=\neg x_{i} \\
\Phi_{S}: \mathbf{K}(\mathbf{X}) \times \mathbf{K}(\mathbf{Y}) \rightarrow \mathbf{X} \cup \mathbf{Y} & \Phi_{S}\left(\mathrm{x}_{\mathrm{i}}, \mathrm{y}_{\mathrm{j}}\right)= \begin{cases}\top & \text { if }(i, j) \in E \\
\perp & \text { if }(i, j) \notin E\end{cases}
\end{array}
$$

Then, with $\Phi_{R S}$ as for $P_{2.2}$, sub-queries $Q_{S}-Q_{R}$ and $Q_{R S}$ are $\mathcal{Q}^{B}$-queries and satisfy

$$
\operatorname{red}_{B}\left(Q_{S}-Q_{R}\right)=\mathbf{K}(\mathbf{Y})\left|\Phi_{R S} \quad \operatorname{red}_{B}\left(Q_{R S}\right)=\mathbf{K}(\mathbf{Y})\right| \Phi_{R S}
$$

The remainder of this reduction is identical to the case of $P_{2.2}$.

Pattern $P_{3.4}$. This case is identical to the case of $P_{2.4}$ where we use the annotation functions $\Phi_{R}$ and $\Phi_{S}$ for the pattern $P_{3.2}$.

5.2.4. Reduction for patterns $P_{4.3}$ and $P_{4.4}$. For queries matching the patterns $P_{4.3}$ or $P_{4.4}$, it is only possible to directly encode the $2 \mathrm{DNF}$ formula $\Psi$ as a database $\mathcal{D}$ such that the annotation of $Q(\mathcal{D})$ is exactly $\Psi$, if the polarity of $O p_{2}$ is odd in $Q_{R T}$. In the case of even polarity, we show that we can derive a database $\mathcal{D}$ and another formula $\Upsilon$ from $\Psi$ such that $P_{Q(\mathcal{D})}=P_{\Upsilon}$ and linearly many calls to an oracle for $P_{\Upsilon}$ suffice to determine $\# \Psi$.

Let $\Psi=\bigvee_{(i, j) \in E} x_{i} y_{j}=\psi_{1} \vee \cdots \vee \psi_{|E|}$ be a $2 \mathrm{DNF}$ formula with $|E|$ clauses over disjoint variable sets $\mathbf{X}$ and $\mathbf{Y}$. Let $\Theta$ be the set of assignments of variables $\mathbf{X} \cup \mathbf{Y}$. Then the number of models of $\Psi$ is defined by $\# \Psi=\sum_{\theta \in \Theta: \theta \models \Psi} 1$. If we partition $\Theta$ into disjoint sets $\Theta_{0} \cup \cdots \cup \Theta_{|E|}$, such that $\theta \in \Theta_{i}$ if and only if $\theta$ satisfies exactly $i$ clauses of $\Psi$, then this sum can be equivalently written as

$$
\# \Psi=\sum_{\theta \in \Theta_{1}: \theta \models \Psi} 1+\cdots+\sum_{\theta \in \Theta_{|E|}: \theta \mid=\Psi} 1=\left|\Theta_{1}\right|+\cdots+\left|\Theta_{|E|}\right| .
$$




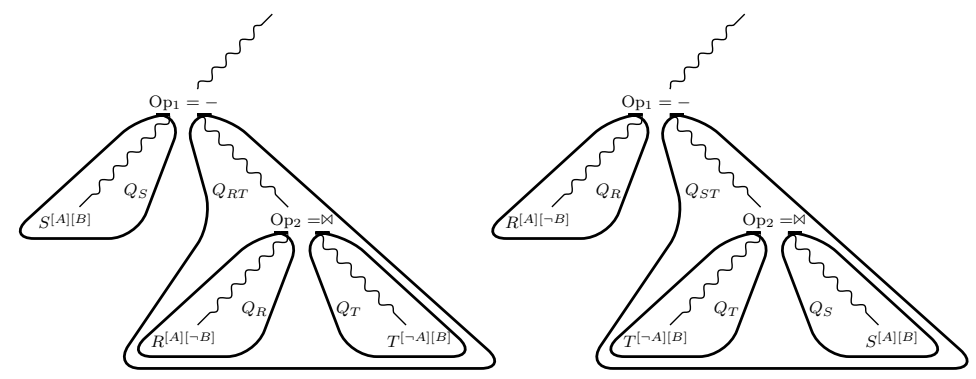

Fig. 13. Schematic illustration of a query that is an annotation-preserving match of pattern $P_{4.3}$ (left) or $P_{5.3}$ (right). A curly path indicates that other operators may occur on it.

We next show how to compute $\left|\Theta_{i}\right|$ using an oracle for $P_{\Upsilon}$, with $\Upsilon$ defined below. Let $\mathbf{Z}=\left\{z_{1}, \ldots, z_{|E|}\right\}$ be a set of variables disjoint from $\mathbf{X} \cup \mathbf{Y}$ and define $\Upsilon$ as

$$
\Upsilon=\bigvee_{i=1}^{|E|} \neg z_{i} \wedge \neg \psi_{i} \quad \text { or, equivalently } \quad \neg \Upsilon=\bigwedge_{i=1}^{|E|}\left(z_{i} \vee \psi_{i}\right)
$$

We fix the probabilities of variables in $\mathbf{X}$ and $\mathbf{Y}$ to $1 / 2$ and of variables in $\mathbf{Z}$ to $p_{z} \in[0,1]$. The probability $1-P_{\Upsilon}=P_{\neg \Upsilon}$ can be expressed by conditioning on the number of satisfied clauses of $\Psi$ :

$$
\begin{aligned}
P_{\neg \Upsilon} & =\sum_{k=0}^{|E|} \underbrace{P\left(\neg \Upsilon \begin{array}{l}
\text { exactly } k \text { clauses } \\
\text { of } \Psi \text { are satisfied }
\end{array}\right)}_{p_{z}^{|E|-k}} \cdot \underbrace{P\left(\begin{array}{c}
\text { exactly } k \text { clauses } \\
\text { of } \Psi \text { are satisfied }
\end{array}\right)}_{\frac{1}{2}^{|\mathbf{X}|+|\mathbf{Y}|} \cdot\left|\Theta_{k}\right|} \\
& =\frac{1}{2}{ }^{|\mathbf{X}|+|\mathbf{Y}|} \sum_{k=0}^{|E|} p_{z}^{|E|-k}\left|\Theta_{k}\right|
\end{aligned}
$$

Intuitively, the first term simplifies to $p_{z}^{|E|-k}$, because if exactly $k$ clauses $\psi_{i}$ are satisfied in $\neg \Upsilon$, then in order to satisfy the remaining $|E|-k$ clauses $\left(z_{i} \vee \psi_{i}\right)$ at least $|E|-k$ of the $z_{i}$ must be satisfied, and this occurs with probability $p_{z}^{|E|-k}$. This is a polynomial in $p_{z}$ of degree $|E|$, with coefficients $\left|\Theta_{0}\right|, \ldots,\left|\Theta_{|E|}\right|$. The $|E|+1$ coefficients can be derived from $|E|+1$ pairs $\left(p_{z}, P_{\Upsilon}\right)$ using Lagrange's polynomial interpolation formula. We conclude that $|E|+1$ oracle calls to $P_{\Upsilon}$ suffice to determine $\# \Psi=\sum_{i=0}^{|E|}\left|\Theta_{i}\right|$.

It remains to show how $\Upsilon$ can be encoded as the annotation of a query that is an annotation-preserving match of one of the patterns $P_{4.3}$ and $P_{4.4}$; given this encoding, any algorithm that evaluates $P_{Q(\mathcal{D})}$ constitutes the above oracle. We give encodings for the two patterns $P_{4.3}$ and $P_{4.4}$ separately.

Pattern $P_{4.3}$. We use the illustration of a query matching $P_{4.3}$ in Fig. 13 (left). By Definition 5.7, a query $Q$ that is an annotation-preserving match of $P_{4.3}$ satisfies the following structural constraint: If $O p_{1}$ is a right descendant of a difference operator, then this operator does not export $[A]$ or $[B]$. Furthermore, attributes $[A]$ and $[B]$ are exported by every operator on the paths from $S$ to $R$ and from $S$ to $T$, respectively.

We distinguish two cases depending on the polarity of $O p_{2}$ in the sub-query $Q_{R T}$ : If the polarity is odd, we can use a filling similar to that of pattern $P_{1.1}$; if it is even we fill to obtain formula $\Upsilon$ as outlined above. 
Case 1: Odd polarity $\left(\operatorname{pol}\left(Q_{R T}, O p_{2}\right)=1\right)$. We fill $R, S, T$ like in the case of pattern $P_{1.1}$ such that $Q_{R}$ is a $\mathcal{Q}^{A}$-query, $Q_{T}$ is a $\mathcal{Q}^{B}$-query, and $Q_{S}$ is a $\mathcal{Q}^{A B}$-query, and the annotation functions are as follows:

$$
\begin{aligned}
\Phi_{R} & : \mathbf{K}(\mathbf{X}) \cup \mathbf{K}(\mathbf{X}) \times \mathbf{K}(\mathbf{Y}) \rightarrow \mathbf{X} \cup \mathbf{Y} \\
\Phi_{S} & : \mathbf{K}(\mathbf{X}) \times \mathbf{K}(\mathbf{Y}) \rightarrow \mathbf{X} \cup \mathbf{Y} \\
\Phi_{T} & : \mathbf{K}(\mathbf{Y}) \cup \mathbf{K}(\mathbf{Y}) \times \mathbf{K}(\mathbf{X}) \rightarrow \mathbf{X} \cup \mathbf{Y} \\
\Phi_{R T} & : \mathbf{K}(\mathbf{X}) \times \mathbf{K}(\mathbf{Y}) \rightarrow \mathbf{X} \cup \mathbf{Y} \\
\Phi_{R S T} & : \mathbf{K}(\mathbf{X}) \times \mathbf{K}(\mathbf{Y}) \rightarrow \mathbf{X} \cup \mathbf{Y}
\end{aligned}
$$

$$
\begin{aligned}
\Phi_{R}\left(\mathrm{x}_{\mathrm{i}}\right) & =\Phi_{R}\left(\mathrm{x}_{\mathrm{i}}, \mathrm{y}_{\mathrm{j}}\right)=x_{i} \\
\Phi_{S}\left(\mathrm{x}_{\mathrm{i}}, \mathrm{y}_{\mathrm{j}}\right) & = \begin{cases}\top & \text { if }(i, j) \in E \\
\perp & \text { if }(i, j) \notin E\end{cases} \\
\Phi_{T}\left(\mathrm{y}_{\mathrm{j}}\right) & =\Phi_{T}\left(\mathrm{y}_{\mathrm{j}}, \mathrm{x}_{\mathrm{i}}\right)=y_{j} \\
\Phi_{R T}\left(\mathrm{x}_{\mathrm{i}}, \mathrm{y}_{\mathrm{j}}\right) & =x_{i} y_{j} \\
\Phi_{R S T}\left(\mathrm{x}_{\mathrm{i}}, \mathrm{y}_{\mathrm{j}}\right) & = \begin{cases}x_{i} y_{j} & \text { if }(i, j) \in E \\
\perp & \text { if }(i, j) \notin E\end{cases}
\end{aligned}
$$

In other words, $R$ consists of a tuple with $A$-value $\mathrm{x}_{\mathrm{i}}$ and annotation $x_{i}$ for each variable $x_{i} \in \mathbf{X}$ that occurs in $\Psi ; T$ consists of a tuple with $B$-value $\mathrm{y}_{j}$ and annotation $y_{j}$ for each variable $y_{j} \in \mathbf{Y}$ that occurs in $\Psi ; S$ consists of a tuple with $(A, B)$-values $\left(\mathrm{x}_{\mathrm{i}}, \mathrm{y}_{\mathrm{j}}\right)$ and annotation $T$ for each clause $x_{i} y_{j}$ in $\Psi$. Recall that we turn variables to constants when used for attributes in relations. For the remaining relations, we distinguish two cases: (1) Any relation that appears on the right side of a difference operator different from $\mathrm{Op}_{1}$ and $\mathrm{Op}_{2}$, is set to $\emptyset$. (2) Any relation with an attribute in $[A]$ and no attribute in $[B]$ is filled like $R$, but with annotation $T$. Symmetrically, any relation with an attribute in $[B]$ and no attribute in $[A]$ is filled like $T$, but with annotation $T$. Relations with attributes in both $[A]$ and $[B]$ become the Cartesian product of $\mathbf{K}(\mathbf{X})$ and $\mathbf{Y}$ and annotation $T$. Any attribute that is neither in $[A]$ nor in $[B]$ is filled with the constant $\square$.

Since the operator $O p_{2}$ has odd polarity in $Q_{R T}$, and since both $[A]$ and $[B]$ are exported by every operator on the path between $O p_{1}$ and $O p_{2}, Q_{R T}$ is a $\mathcal{Q}^{A B}$-query with annotations

$$
\operatorname{red}_{A B}\left(Q_{R T}\right)=\mathbf{K}(\mathbf{X}) \times \mathbf{K}(\mathbf{Y}) \mid \neg \Phi_{R T} .
$$

Then, $Q_{S}-Q_{R T}$ is a $\mathcal{Q}^{A B}$ query populated as follows:

$$
\begin{aligned}
\operatorname{red}_{A B}\left(Q_{S}-Q_{R T}\right) & =\mathbf{K}(\mathbf{X}) \times \mathbf{K}(\mathbf{Y}) \mid \Phi_{R S T} . \\
\Phi_{R S T}\left(\mathrm{x}_{i}, \mathrm{y}_{\mathrm{j}}\right) & = \begin{cases}x_{i} y_{j} & \text { if }(i, j) \in E \\
\perp & \text { if }(i, j) \notin E .\end{cases}
\end{aligned}
$$

The final projection $\pi_{-[A]-[B]}$ yields one answer tuple, whose annotation is the disjunction of all clauses in $\Psi$.

Case 2: Even polarity $\left(\operatorname{pol}\left(Q_{R T}, O p_{2}\right)=0\right)$. We encode the formula $\Upsilon$ from Equation (13) using the above annotation functions adjusted as follows:

$$
\begin{aligned}
\Phi_{S}: \mathbf{K}(\mathbf{X}) \times \mathbf{K}(\mathbf{Y}) \rightarrow \mathbf{X} \cup \mathbf{Y} & \Phi_{S}\left(\mathrm{x}_{\mathrm{i}}, \mathrm{y}_{\mathrm{j}}\right)= \begin{cases}\neg z_{k} & \text { if } x_{i} y_{j} \text { is a clause } \psi_{k} \text { in } \Psi \\
\perp & \text { else }\end{cases} \\
\Phi_{R S T}: \mathbf{K}(\mathbf{X}) \times \mathbf{K}(\mathbf{Y}) \rightarrow \mathbf{X} \cup \mathbf{Y} \quad \Phi_{R S T}\left(\mathrm{x}_{\mathrm{i}}, \mathrm{y}_{\mathrm{j}}\right) & = \begin{cases}\neg z_{k} \wedge \neg \psi_{k} & \text { if } x_{i} y_{j} \text { is a clause } \psi_{k} \text { in } \Psi \\
\perp & \text { else }\end{cases}
\end{aligned}
$$

The sub-queries $Q_{R T}$ and $Q_{S}-Q_{R T}$ are then populated as follows:

$$
\begin{aligned}
\operatorname{red}_{A B}\left(Q_{R T}\right) & =\mathbf{K}(\mathbf{X}) \times \mathbf{K}(\mathbf{Y}) \mid \Phi_{R T} \\
\operatorname{red}_{A B}\left(Q_{S}-Q_{R T}\right) & =\mathbf{K}(\mathbf{X}) \times \mathbf{K}(\mathbf{Y}) \mid \Phi_{R S T}
\end{aligned}
$$


since $O p_{1}$ has even polarity in $Q_{R T}$. As before, the final projection $\pi_{-[A]-[B]}$ yields one answer tuple whose annotation is the disjunction of the annotation of $Q_{S}-Q_{R T}$ which is exactly $\Upsilon$, cf. Equation 13 .

Pattern $P_{4.4}$. The reduction is equivalent to the case of pattern $P_{4.3}$ when the sign of the annotation functions $\Phi_{T}$ is flipped regardless of the polarity pol $\left(Q_{R T}, O p_{2}\right)$ of $O p_{2}$ in $Q_{R T}$.

5.2.5. Reduction for patterns $P_{5.3}$ and $P_{5.4}$. By Definition 5.7, a query $Q$ that has an annotation-preserving match with one of $P_{5.3}$ and $P_{5.4}$ if $O p_{1}$ is a right descendant of a difference operator, then this operator does not export $[A]$ or $[B]$.

Pattern $P_{5.3}$. Figure 13 (right) gives a schematic illustration of a query matching $P_{5.3}$. We distinguish two cases depending on whether $[B]$ is or is not exported by $O p_{1}$.

$[B]$ is exported by $O p_{1}$. Without loss of generality, assume that $O p_{1}$ is the first operator that allows for a match by virtue of Lemma 5.8 Then $Q_{R}$ contains a relation $X$ that exports $[B]$ and is joined with $R$ in $Q_{R}$. If this relation is $X^{[A][B]}$, then $Q$ is an annotation-preserving $(R, X, T)$-match of one of the patterns $P_{2 . *}$ or $P_{3 . *}$; if this relation is $X^{[\neg A][B]}$, then $Q$ is an annotation-preserving $(R, S, X)$-match of one of the patterns $P_{1 . *}$.

$[B]$ is not exported by $O p_{1}$. The sub-query $Q_{S T}$ contains a projection operator $O p_{\pi}=$ $\pi_{-[B]}$ such that every operator between $O p_{\pi}$ and $O p_{1}$ exports $[A]$ but not $[B]$, and every operator between $O p_{\pi}$ and $O p_{2}$ exports $[A]$ and $[B]$. Let $Q_{\pi}$ be the sub-query rooted at $O p_{\pi}$. We first show that one may assume without loss of generality that $O p_{2}$ is left-deep in $Q_{\pi}$. Assume to the contrary that there is a difference operator $O p_{-}$between $O p_{\pi}$ and $O p_{2}$ that has $O p_{2}$ as a right descendant; clearly, $O p_{-}$exports $[A]$ and $[B]$ and hence its left subquery contains relations $X^{[A][\neg B]}$ and $Y^{[\neg A][B]}$ or it contains a relation $Z^{[A][B]}$. In the former case, $Q$ is an annotation-preserving $(R, S, Y)$-match of pattern $P_{5.4}$; in the latter case, $Q$ is an annotation-preserving $(R, Z, T)$-match of pattern $P_{6.4}$. In both cases, the new operator $\mathrm{Op}_{2}$ is left-deep in $Q_{\pi}$. Within this second case, we analyse two sub-cases depending on the polarity of $O p_{\pi}$ in $Q_{S T}$ :

Case 1: Even polarity $\left(\operatorname{pol}\left(Q_{S T}, O p_{\pi}\right)=0\right)$. Let $\mathbf{V}=\mathbf{X} \cup \mathbf{Y}$ and $\mathbf{N}=\{1, \ldots,|E|\}$ be the set of indices of $\Psi^{\prime} s$ clauses: $\Psi=\psi_{1} \vee \cdots \vee \psi_{|E|}$. We use the following annotation functions:

$$
\begin{array}{rlrl}
\Phi_{R} & : \mathbf{N} \rightarrow \mathbf{V} & \Phi_{R}(i) & =\top \\
\Phi_{S} & : \mathbf{N} \times \mathbf{K}(\mathbf{V}) \rightarrow \mathbf{V} & \Phi_{S}(i, \mathrm{v}) & = \begin{cases}\top & \text { if clause } \psi_{i} \text { contains variable } v \\
\perp & \text { else }\end{cases} \\
\Phi_{T} & : \mathbf{K}(\mathbf{V}) \cup \mathbf{K}(\mathbf{V}) \times \mathbf{N} \rightarrow \mathbf{V} & \Phi_{T}(\mathrm{v}) & =\Phi_{T}(\mathrm{v}, i)=\neg v \\
\Phi_{S T} & : \mathbf{N} \times \mathbf{K}(\mathbf{V}) \rightarrow \mathbf{V} & \Phi_{S T}(i, \mathrm{v}) & = \begin{cases}\neg v & \text { if clause } \psi_{i} \text { contains variable } v \\
\perp & \text { else }\end{cases} \\
\Phi_{\pi S T}: \mathbf{N} \rightarrow \mathbf{V} & \Phi_{\pi S T}(i) & =\neg \psi_{i} \\
\Phi_{R S T} & : \mathbf{N} \rightarrow \mathbf{V} & \Phi_{R S T}(i) & =\psi_{i}
\end{array}
$$

That is, we set relation $R$ to contain a tuple $(n)$ annotated with $T$ for every clause with index $n \in \mathbf{N}$. Relation $S$ contains all tuples $(n, \mathrm{v})$ where $n \in \mathbf{N}$ is a clause index and $\mathrm{v} \in \mathbf{K}(\mathbf{V})$ is the constant corresponding to the variable $v \in \mathbf{V} ;(n, \mathrm{v})$ is annotated with $\top$ if clause with index $n$ contains variable $v$, and with $\perp$ otherwise. Relation $T$ has a tuple (v) annotated with $\neg v$ for each variable $v$ in $\Psi$. The annotations of relations $R, S, T$ account for their respective polarity in $Q_{R}, Q_{S}, Q_{T}$. The sub-query $Q_{T} \bowtie Q_{S}$ is a $\mathcal{Q}^{A B}$-relation:

$$
\operatorname{red}_{A B}\left(Q_{T} \bowtie Q_{S}\right)=\mathbf{N} \times \mathbf{K}(\mathbf{V}) \mid \Phi_{S T}
$$




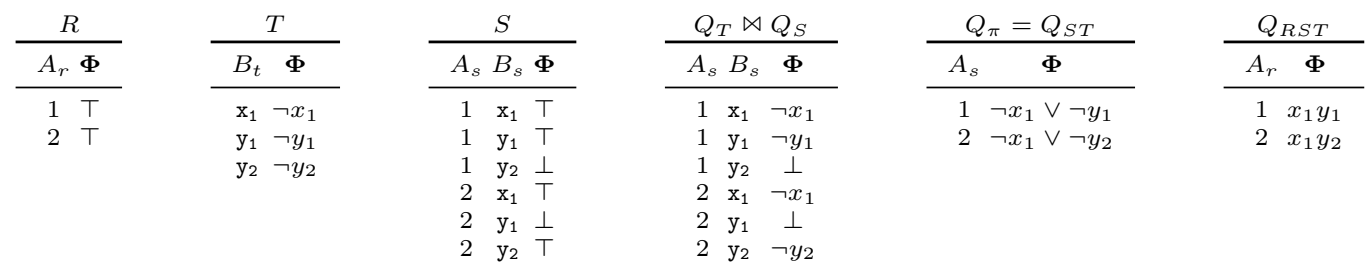

Fig. 14. Relations $R, S, T$ for the hardness reduction of a query that is an annotation-preserving match of pattern $P_{5.3}$ where (1) $O p_{1}$ does not export $[B]$ and (2) the projection operator $\pi_{-[B]}$ on the path between $O p_{1}$ and $O p_{2}$ has even polarity in $Q_{S T}$ (the sub-query containing both relations $S$ and $T$ ). Only attributes $[A]$ and $[B]$ are depicted, and it is assumed that $R, S, T$ have even polarity in their respective sub-queries $Q_{R}, Q_{S}$, and $Q_{T}$. The database is with respect to the formula $\Psi=\psi_{1} \vee \psi_{2}=x_{1} y_{1} \vee x_{1} y_{2}$.

The operator $O p_{\pi}$ turns the $\mathcal{Q}_{A B}$-relation $Q_{T} \bowtie Q_{S}$ into a $\mathcal{Q}^{A}$-relation $Q_{\pi}$. Since $O p_{\pi}$ has even polarity in $Q_{S T}$, this annotation can be preserved for $Q_{S T}$ :

$$
\operatorname{red}_{A}\left(Q_{\pi}\right)=\mathbf{N}\left|\Phi_{\pi S T} \quad \operatorname{red}_{A}\left(Q_{S T}\right)=\mathbf{N}\right| \Phi_{\pi S T} .
$$

Finally, the annotations of $R$ can be preserved in $Q_{R}$. The sub-query $Q_{R}-Q_{S T}$ flips the sign of the annotations of $Q_{S T}$. This yields

$$
\operatorname{red}_{A}\left(Q_{R}\right)=\mathbf{N}\left|\Phi_{R} \quad \operatorname{red}_{A}\left(Q_{R S T}\right)=\mathbf{N}\right| \Phi_{R S T} .
$$

As in the previous cases, the final projection $\pi_{-[A]}$ yields a nullary relation whose only tuple is annotated with $\neg^{\mathrm{pol}\left(Q, O p_{1}\right)} \Psi$.

Example 5.11. Figure 14 shows how $R, S$, and $T$ are filled for the formula $\Psi=x_{1} y_{1} \vee x_{1} y_{2}$ and a query matching the pattern $P_{5.3}$, and how these annotations are propagated through the query operators.

Case 2: Odd polarity $\left(\operatorname{pol}\left(Q_{S T}, O p_{\pi}\right)=1\right)$. The number of difference operators between the root of the query and the relations $S$ and $T$ is even. For the annotation of the query, these operators act equivalently to a sequence of join operators: We fill the relations such that $Q_{T}$ is a $\mathcal{Q}^{B}$-query, $Q_{S}$ is a $\mathcal{Q}^{A B}$-query, $Q_{R}$ is a $\mathcal{Q}^{A}$-query, and then $Q_{S T}$ is a $\mathcal{Q}^{A}$-query, where the annotation functions for $R, S$, and $T$ are as for the pattern $P_{1.1}$, that is:

$$
\begin{aligned}
& \Phi_{R}: \mathbf{K}(\mathbf{X}) \cup \mathbf{K}(\mathbf{X}) \times \mathbf{K}(\mathbf{Y}) \rightarrow \mathbf{X} \cup \mathbf{Y} \\
& \Phi_{S}: \mathbf{K}(\mathbf{X}) \times \mathbf{K}(\mathbf{Y}) \rightarrow \mathbf{X} \cup \mathbf{Y} \\
& \Phi_{T}: \mathbf{K}(\mathbf{Y}) \cup \mathbf{K}(\mathbf{Y}) \times \mathbf{K}(\mathbf{X}) \rightarrow \mathbf{X} \cup \mathbf{Y} \\
& \Phi_{S T}: \mathbf{K}(\mathbf{X}) \times \mathbf{K}(\mathbf{Y}) \rightarrow \mathbf{X} \cup \mathbf{Y} \\
& \Phi_{\pi S T}: \mathbf{K}(\mathbf{X}) \rightarrow \mathbf{X} \cup \mathbf{Y} \\
& \Phi_{R S T}: \mathbf{K}(\mathbf{X}) \rightarrow \mathbf{X} \cup \mathbf{Y}
\end{aligned}
$$

$$
\begin{aligned}
& \Phi_{R}\left(\mathrm{x}_{\mathrm{i}}\right)=\Phi_{R}\left(\mathrm{x}_{\mathrm{i}}, \mathrm{y}_{\mathrm{j}}\right)=x_{i} \\
& \Phi_{S}\left(\mathrm{x}_{\mathrm{i}}, \mathrm{y}_{\mathrm{j}}\right)= \begin{cases}\top & \text { if }(i, j) \in E \\
\perp & \text { if }(i, j) \notin E\end{cases} \\
& \Phi_{T}\left(\mathrm{y}_{\mathrm{i}}\right)=\Phi_{T}\left(\mathrm{y}_{\mathbf{j}}, \mathrm{x}_{\mathrm{i}}\right)=y_{i} \\
& \Phi_{S T}\left(\mathrm{x}_{i}, \mathrm{y}_{j}\right)= \begin{cases}y_{j} & \text { if }(i, j) \in E \\
\perp & \text { if }(i, j) \notin E\end{cases} \\
& \Phi_{\pi S T}\left(\mathrm{x}_{\mathrm{i}}\right)=\bigvee_{(i, j) \in E} y_{j} \\
& \Phi_{R S T}\left(\mathrm{x}_{\mathrm{i}}\right)=x_{i} \wedge \bigvee_{(i, j) \in E} y_{j}
\end{aligned}
$$


and obtain the following reductions

$$
\begin{aligned}
\operatorname{red}_{A}\left(Q_{R}\right) & =\mathbf{K}(\mathbf{X}) \mid \Phi_{R} & \operatorname{red}_{A B}\left(Q_{T} \bowtie Q_{S}\right) & =\mathbf{K}(\mathbf{X}) \times \mathbf{K}(\mathbf{Y}) \mid \Phi_{S T} \\
\operatorname{red}_{A B}\left(Q_{S}\right) & =\mathbf{K}(\mathbf{X}) \times \mathbf{K}(\mathbf{Y}) \mid \Phi_{S} & \operatorname{red}_{A}\left(Q_{\pi}\right) & =\mathbf{K}(\mathbf{X}) \mid \Phi_{\pi S T} \\
\operatorname{red}_{B}\left(Q_{T}\right) & =\mathbf{K}(\mathbf{Y}) \mid \Phi_{T} & \operatorname{red}_{A}\left(Q_{S T}\right) & =\mathbf{K}(\mathbf{X}) \mid \neg \Phi_{\pi S T}
\end{aligned}
$$

where the sign of the annotations of $\operatorname{red}_{A}\left(Q_{\pi}\right)$ and $\operatorname{red}_{A}\left(Q_{S T}\right)$ is flipped because $O p_{\pi}$ has odd polarity in $Q_{S T}$. This yields

$$
\operatorname{red}_{A}\left(Q_{R S T}\right)=\mathbf{K}(\mathbf{X}) \mid \Phi_{R S T}
$$

for the sub-query rooted at $O p_{1}=-$ and the annotation $\neg^{\text {pol }\left(Q, O p_{1}\right)} \Psi$ for the query $Q$.

Pattern $P_{5.4}$. The analysis of the pattern $P_{5.4}$ is analogous to the case of the pattern $P_{5.3}$, where the sign of the annotation function $\Phi_{S}$ is flipped.

5.2.6. Reduction for the pattern $P_{6.4}$. The analysis of pattern $P_{6.4}$ is analogous to the case of pattern $P_{5.3}$, where the sign of the annotation function $\Phi_{T}$ is flipped.

\section{THE TRACTABILITY FRONTIER FOR QUANTIFIED QUERIES}

This section investigates the data complexity of the probabilistic query evaluation problem for quantified queries that express binary relationships amongst sets of entities: set division, set inclusion, set equality, set difference, and set incomparability. For example, a set-inclusion query could find non-critical overseas suppliers, that is, overseas suppliers for parts that also have domestic suppliers. A set-division query could find all suppliers for a given set of items. These queries can be expressed in relational algebra using nested negation and repeated relation symbols, as shown in Figs. 15 and 5. We analyse the data complexity of their exact computation on tuple-independent databases: For tractable queries, we give an explicit $\mathcal{O}(|\mathcal{D}|)$-algorithm for computing the probability of the query annotation based on Shannon expansion and the inclusion-exclusion principle; for intractable queries, we give a hardness reduction from \#2DNF.

Although we only discuss a handful of quantified queries, each of them can in fact represent an entire class by taking as input relations independent hierarchical 1RA ${ }^{-}$queries, such that for each such query $Q$ all of its exported attributes are root in $Q$ (that is, for a root attribute $A$, each relation in $Q$ has an attribute in the class [A]). This holds since the result of $Q$ on a tuple-independent database is again a tuple-independent database.

\subsection{Tractable quantified queries}

Assume we are given a tuple-independent relation $S$ with schema $S$ (sid, item) that specifies pairs of set identifiers and items in these sets. We would like to compute the pairs of set identifiers $\left(s_{1}, s_{2}\right)$ and their probabilities, such that $s_{1}$ is included in/strictly included in/equal to/incomparable with $s_{2}$. The corresponding queries are denoted by $S_{\subseteq}, S_{\subset}, S_{=}$, and $S_{<>}$respectively, and defined in Fig. 15 the queries $S_{d=}$ and $S_{d \subseteq}$ are the restrictions of $S_{=}$and $S_{\subseteq}$ to pairs of different set identifiers. The set-division quantified query is given in Fig. 5 .

Example 6.1. Relation $S$ from Fig. 15 defines three uncertain subsets of $\{a, b, c\}: s_{1}=$ $\{a, b, c\}, s_{2}=\{a, b\}$, and $s_{3}=\{a, c\}$. In the absence of uncertainty, we have that $s_{2} \subset s_{1}$ and $s_{3} \subset s_{1}$, and $s_{2}$ and $s_{3}$ are incomparable. Under the possible worlds semantics, however, further relationships may hold between the sets. For instance, the set $s_{1}$ is included in $s_{2}$ for those assignments of the random variables that satisfy the annotation associated with $(1,2)$ in $S_{\subseteq}$. This annotation reads as follows. If $a$ or $b$ are in $s_{1}$, then they must also be in $s_{2}$; this is expressed by the term $\neg\left(x_{1} \neg y_{1} \vee x_{2} \neg y_{2}\right)$. If $c$ is in $s_{1}$, then $(1,2)$ may not be in $S_{\subseteq}$; this is expressed by $\neg x_{3}$. The disjunctions $x_{1} \vee x_{2} \vee x_{3}$ and $y_{1} \vee y_{2}$ ensure that the two sets have at least one item and are thus recorded in $S$. The direct application of 


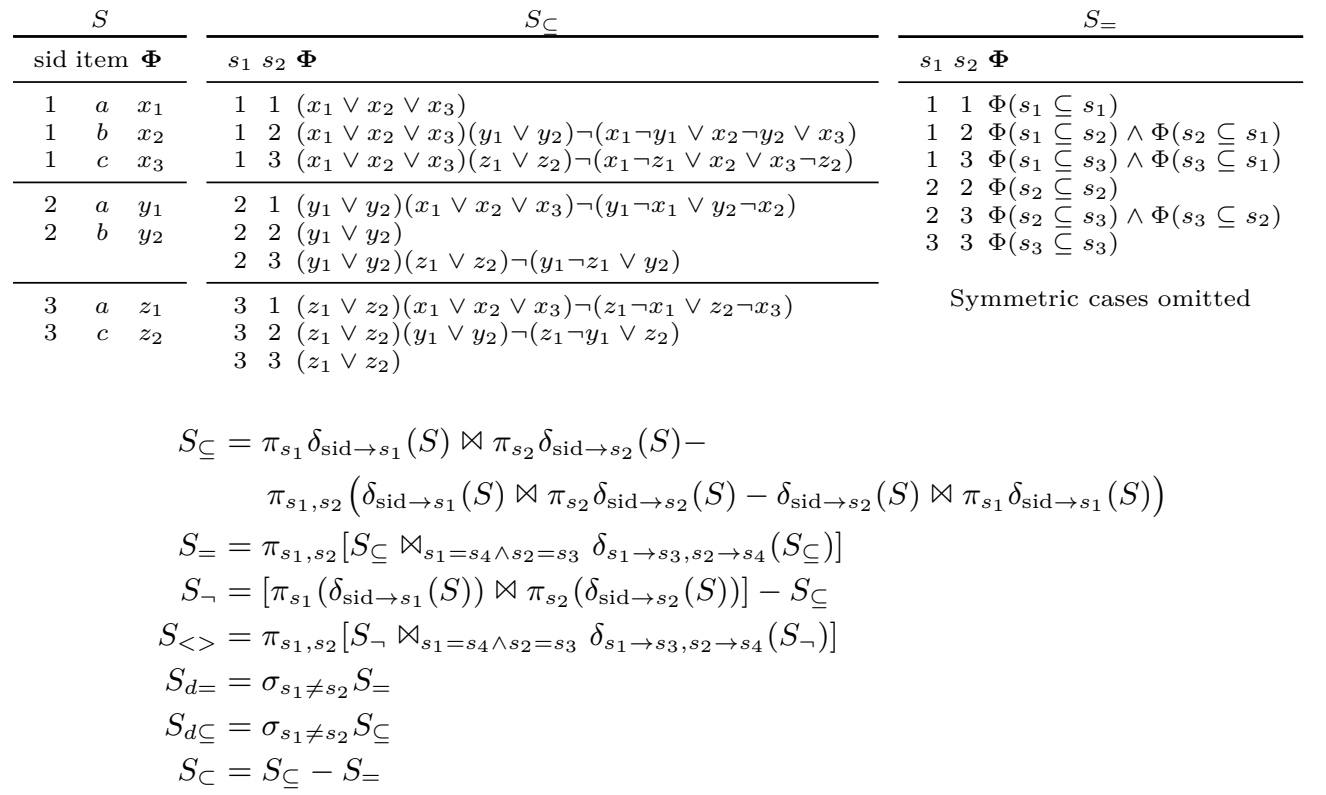

Fig. 15. Definition of queries for computing set inclusion, equality, and incomparability. The tables show an example database $(S)$ and the result of the set inclusion $\left(S_{\subseteq}\right)$ and equality $\left(S_{=}\right)$queries. In the table for $S_{=}, \Phi\left(s_{i} \subseteq s_{j}\right)$ is the annotation of the tuple $(i, j)$ in relation $S_{\subseteq}$.

the translation $\llbracket S_{\subset} \rrbracket$ according to Algorithm 1 yields an equivalent yet syntactically slightly different annotation than that depicted in Fig. 15

Remark 6.2. Since a set is equal to itself, one would expect that $(i, i)$ occurs in $S=$ with probability 1 for all sets $i$ from $S$. However, as shown in Fig. 15 , the annotation of $(1,1) \in S_{=}$ is $x_{1} \vee x_{2} \vee x_{3}$, whose probability is not always 1 . This is correct due to the closed world assumption in relational databases: In the worlds in which the annotation is false, there is no set 1 and hence the set-inclusion query cannot produce pairs involving this set.

The probability that a pair of set identifiers is in the answer to any of our quantified queries can be computed efficiently.

THEOREM 6.3. Let $S$ be a tuple-independent relation over schema $S$ (sid, item) that defines sets and their items, and $S_{\subseteq}, S_{d \subseteq}, S_{\subset}, S_{=}, S_{d=}$, and $S_{<>}$be the quantified queries defined in Fig. 15. Any tuple in the answer to these queries has an annotation of size $\mathcal{O}(|S|)$ and its probability can be computed in time $\mathcal{O}(|S|)$.

Proof. We compute the probabilities of the annotations for the query $S_{\subseteq}$ using recurrences and Shannon expansion. The annotations associated with tuples in $\bar{S}_{\subseteq}$ (cf. Example 6.1) have the general form

$$
\left(x_{1} \vee \ldots \vee x_{m}\right)\left(y_{1} \vee \ldots \vee y_{n}\right) \neg\left(x_{1} \neg y_{1} \vee \ldots \vee x_{k} \neg y_{k} \vee x_{k+1} \vee \ldots \vee x_{m}\right),
$$

where $k$ represents the number of items the two sets have in common, and $m-k$ is the number of items in the first set and not in the second. This is equivalent to $\left(x_{1} \vee \ldots \vee\right.$ $\left.x_{k}\right)\left(y_{1} \vee \ldots \vee y_{n}\right) \neg\left(x_{1} \neg y_{1} \vee \ldots \vee x_{k} \neg y_{k}\right) \neg\left(x_{k+1} \vee \ldots \vee x_{m}\right)$, and since the variables in the last negated disjunction occur only once, we can compute the probability of this disjunction efficiently and separately from the rest. We are thus left with $\left(x_{1} \vee \ldots \vee x_{k}\right)\left(y_{1} \vee \ldots \vee\right.$ 


$$
\begin{aligned}
\left.y_{n}\right) \neg\left(x_{1} \neg y_{1} \vee \ldots \vee x_{k} \neg y_{k}\right) . \text { Let } & \\
\Sigma_{i}^{x y} & =\neg\left(x_{i} \neg y_{i} \vee \ldots \vee x_{k} \neg y_{k}\right), \\
\Sigma_{i}^{x, x y} & =\left(x_{i} \vee \ldots \vee x_{k}\right) \Sigma_{i}^{x y}, \\
\Sigma_{i}^{x, y, x y} & =\left(y_{i} \vee \ldots \vee y_{n}\right) \Sigma_{i}^{x, x y} .
\end{aligned}
$$

We then have the following for any $i$ with $1 \leq i<k$ :

$$
\begin{aligned}
\Sigma_{i}^{x y} & =\left(x_{i} y_{i} \vee \neg x_{i}\right) \Sigma_{i+1}^{x y} \\
\Sigma_{i}^{x, x y} & =x_{i} y_{i} \Sigma_{i+1}^{x y} \vee \neg x_{i} \Sigma_{i+1}^{x, x y} \\
\Sigma_{i}^{x, y, x y} & =x_{i} y_{i} \Sigma_{i+1}^{x y} \vee \neg x_{i}\left[y_{i} \Sigma_{i+1}^{x, x y} \vee \neg y_{i} \Sigma_{i+1}^{x, y, x y}\right]
\end{aligned}
$$

Each of the above three formulas has a constant number of variables and refers recursively to at most three sub-formulas where one pair of variables $\left(x_{i}, y_{i}\right)$ is removed. The recursion depth is thus bounded by the number of variables in $S$. Given the probabilities for the referred formulas, the probability of each referring formula can be computed efficiently, since all terms in the sums are pairwise mutually exclusive. We thus have $\mathcal{O}(|S|)$ time complexity for probability computation of $\Sigma_{1}^{x, y, x y}$ and of annotations in $S_{\subseteq}$. Similar recurrences can be obtained for $S_{=}$and $S_{<>}$under the same variable order elimination.

We next discuss the case of relational division. In the TPC-H scenario, a useful query with division would find the most likely suppliers for all parts of a given brand, cf. Fig. 5 for a query evaluation example. Similar to set-relation queries, we can use recurrence formulas to obtain a linear-time algorithm for computing the probabilities of tuples in the result of a set-division query.

THEOREM 6.4. Let $T=R \div S$, where $R$ and $S$ are any tuple-independent relations. Then, any tuple in $T$ has an annotation of size $\mathcal{O}(|R|+|S|)$ and its probability can be computed in time $\mathcal{O}(|R|+|S|)$.

Proof. Let $R(\bar{A}, \bar{B})$ and $S(\bar{B})$ be the schemas of $R$ and $S$, respectively. The schema of $T$ is thus $T(\bar{A})$. The following analysis applies separately to each value $\bar{a}$ in $R$.

Let $\left\{y_{1}, \ldots, y_{n}\right\}$ and $\left\{x_{1}, \ldots, x_{m}\right\}$ be the variables associated with tuples in $S$ and with tuples $(\bar{a}, \bar{b})$ in $R$ for a value $\bar{a}$, respectively. The annotation of $\bar{a}$ in $T$ has the form $\left(x_{1} \vee\right.$ $\left.\ldots \vee x_{m}\right) \neg\left(y_{1} \neg x_{1} \vee \ldots \vee y_{k} \neg x_{k} \vee y_{k+1} \vee \ldots \vee y_{n}\right)$, where $k \leq m$ is such that $x_{1}, \ldots, x_{k}$ are those variables associated with tuples $(\bar{a}, \bar{b})$ in $R$ where $\bar{b}$ is in $S$. The term $\neg\left(y_{k+1} \vee\right.$ $\left.\ldots \vee y_{n}\right)$ can be factored out and its probability computed efficiently since it is a sum of independent variables that do not occur elsewhere in the annotation. We are left with $\left(x_{1} \vee \ldots \vee x_{m}\right) \neg\left(y_{1} \neg x_{1} \vee \ldots \vee y_{k} \neg x_{k}\right)$. Let

$$
\begin{aligned}
\Sigma_{i}^{x} & =\left(x_{i} \vee \ldots \vee x_{m}\right) \\
\Sigma_{i}^{x y} & =\neg\left(y_{i} \neg x_{i} \vee \ldots \vee y_{k} \neg x_{k}\right) \\
\Sigma_{i}^{x, x y} & =\Sigma_{i}^{x} \Sigma_{i}^{x y} .
\end{aligned}
$$

Using Shannon expansion, we can decompose them as follows:

$$
\begin{aligned}
\Sigma_{i}^{x} & =x_{i} \vee \neg x_{i} \Sigma_{i+1}^{x} \\
\Sigma_{i}^{x y} & =\left[x_{i} \vee \neg x_{i} \neg y_{i}\right] \Sigma_{i+1}^{x y} \\
\Sigma_{i}^{x, x y} & =x_{i} \Sigma_{i+1}^{x y} \vee \neg x_{i} \neg y_{i} \Sigma_{i+1}^{x, x y} .
\end{aligned}
$$

These recurrence formulas share the properties of those for set-relation queries: Given the probabilities for the referred formulas, the probability of each referring formula can be computed efficiently, since all terms in the sums are pairwise mutually exclusive. Moreover, the referred formulas have at least one variable less than the referring one. 
All recurrence formulas for our quantified queries use the same variable order for Shannon expansion: $x_{1}, y_{1}, \ldots, x_{k}, y_{k}$.

\subsection{Intractable quantified queries}

Some of the queries discussed in Section 6.1 become \#P-hard when one or more of their attributes are projected out.

Theorem 6.5. For any $x \subset\left\{s_{1}, s_{2}\right\}$, the data complexity of the queries $\pi_{\emptyset}(S \div I)$, $\pi_{x}\left(S_{d=}\right), \pi_{x}\left(S_{d \subseteq}\right), \pi_{x}\left(S_{\subset}\right)$, and $\pi_{x}\left(S_{<>}\right)$is \#P-hard.

Proof. The proof is by direct reduction from the model counting problem for 2DNF formulas. We detail the reduction for the case of $\pi_{\emptyset}(S \div I)$, cf. Fig. 5 for its definition and an example; the reductions for the remaining queries are analogous.

Let $\Phi=c_{1} \vee \cdots \vee c_{n}$ be an input 2DNF formula with $n$ clauses. Without loss of generality, we assume that the relation $I$, which specifies set items, is unary and the relation $S$, which specifies sets and their items, is binary. We construct the relation $I$ such that for each variable $v$ in $\Phi$ there is exactly one tuple, or item, $\mathrm{v}$ in $I$ with annotation $\neg v$. We construct the relation $S$ such that there is one distinct set $i$ for each clause $c_{i}$ in $\Phi$ and this set consists of the items corresponding in $I$ to the variables not in $c_{i}$. That is, for each clause $c_{i}, S$ consists of as many tuples as variables that are in $\Phi$ and not in $c_{i}$; one such tuple is a pair of the set $i$ and a constant representing a variable in $\Phi$ but not in $c_{i}$. All tuples in $S$ are annotated with $T$, that is, the relation $S$ is deterministic. By construction, the annotation of the query result becomes $\Phi$.

Although Boolean relational division $\pi_{\emptyset}(S \div I)$ is hard in general, its tractability depends on the input probability distribution in case each item value in $I$ is paired with each set in $S$, as we discuss next. Assume without loss of generality there are $n$ item values $1, \ldots, n$ in $I$ annotated with distinct variables $y_{1}, \ldots, y_{n}$, and there are $m$ sets $1, \ldots, m$ in $S$, such that each set $i$ has $n+k_{i}$ possible tuples $(i, 1), \ldots,\left(i, n+k_{i}\right)$ annotated by $\neg x_{1}^{i}, \ldots, x_{n+k_{i}}^{i}$. Following the annotation pattern in Fig. 55, the annotation of the query becomes:

$$
\Phi=\bigvee_{i=1}^{m}\left(\neg x_{1}^{i} \vee \ldots \vee \neg x_{n+k_{i}}^{i}\right) \neg\left(y_{1} x_{1}^{i} \vee \ldots \vee y_{n} x_{n}^{i}\right)
$$

By negating $\Phi$ and removing redundant terms, we obtain:

$$
\neg \Phi=\left[\bigwedge_{i=1}^{m} \bigwedge_{j=1}^{n+k_{i}} x_{j}^{i}\right] \vee\left[\bigwedge_{i=1}^{m} y_{1} x_{1}^{i} \vee \ldots \vee y_{n} x_{n}^{i}\right]
$$

By applying the inclusion-exclusion principle and simplifying, the probability of $\neg \Phi$ is then:

$$
P(\neg \Phi)=P\left[\bigwedge_{i=1}^{m} \bigwedge_{j=1}^{n+k_{i}} x_{j}^{i}\right]+P\left[\bigwedge_{i=1}^{m} y_{1} x_{1}^{i} \vee \ldots \vee y_{n} x_{n}^{i}\right]-P\left[\bigwedge_{i=1}^{m} \bigwedge_{j=1}^{n+k_{i}} x_{j}^{i}\right] \cdot P\left[y_{1} \vee \cdots \vee y_{n}\right]
$$

The first and the third terms in the sum can be computed trivially regardless of the probability distribution. The second term can be computed efficiently in case of uniform distribution:

Proposition 6.6. The number of models of the propositional formula

$$
\bigwedge_{i=1}^{m} y_{1} x_{1}^{i} \vee \ldots \vee y_{n} x_{n}^{i} \quad \text { is } \quad \sum_{j=1}^{n}\left(\begin{array}{l}
n \\
j
\end{array}\right)\left(2^{n}-2^{n-j}\right)^{m} .
$$


The proof exploits the combinatorial structure of the formula. The formula of Proposition 6.6 admits efficient model counting - and thus probability computation under uniform probability distribution for the variables - due to its symmetry: For any choice of $k$ out of $n$ variables $y_{j}$ set to true, the number of satisfying assignments is the same and only depends on $k, n$, and $m$. In case of arbitrary input probability distributions, however, the formula is no longer symmetric and setting different $k$ variables $y_{j}$ to true can lead to different probabilities. In fact, arbitrary positive bipartite $2 \mathrm{CNF}$ formulas can be obtained by appropriately setting variables $x_{j}^{i}$ to true or false.

\section{BEYOND 1RA- QUERIES}

In this section we discuss the effect of various extensions of $1 \mathrm{RA}^{-}$on query tractability.

A dichotomy for full relational algebra seems unattainable since key reasoning tasks for such queries, such as equivalence, emptiness, or subsumption, are undecidable: Given two equivalent queries, one hard and one tractable, we cannot have an effective procedure that tells us that their union is a tractable query. Restrictions on the use of negation, e.g., guarded negation Bárány et al. 2012], enable decidability of query equivalence and can pave the way to a complexity dichotomy for (possibly repeating) relational queries with guarded negation in probabilistic databases.

\subsection{Non-repeating relational algebra}

If we add the union operator to the language $1 \mathrm{RA}^{-}$, we need a different syntactic characterisation of the tractable queries, since the hierarchical property is not defined for queries with union. An immediate attempt would consider all (union-free) sub-queries obtained by choosing one term at each union and checking whether all of them are hierarchical. This approach fails since such sub-queries are not necessarily $\exists$-consistent. For instance, the nonrepeating relational algebra query $Q=\pi_{\emptyset}\left[S-\left(R \bowtie S_{1} \cup T \bowtie S_{2}\right)\right]$ over database schema $\left(S(A, B), R(A), S_{1}(A, B), T(B), S_{2}(A, B)\right)$ has two hierarchical union-free sub-queries under $\pi_{\emptyset}: \pi_{\emptyset}\left(S-\left(R \bowtie S_{1}\right)\right)$ and $\pi_{\emptyset}\left(S-\left(T \bowtie S_{2}\right)\right)$. However, these sub-queries cannot be rewritten to $\exists$-consistent $\mathrm{RC}^{\exists}$ queries, since they have roots $A$ and $B$ respectively; it can be further shown that $Q$ is \#P-hard.

An alternative characterisation would be to check $\exists$-consistency and the $\mathrm{RC}^{\exists}$-hierarchical property of the $\mathrm{RC}^{\exists}$ expression $Q_{r}$ representing the rewriting of a non-repeating relational algebra query $Q$ described in Section 3.1 Then $Q$ is tractable when $Q_{r}$ is $\exists$-consistent and RC-hierarchical. Checking these properties can be done efficiently in the size of the input $\mathrm{RC}^{\exists}$ query, yet $Q_{r}$ may be much larger than $Q$ (as per discussion at the end of Section 3.1. It is open whether the characterisation of tractable non-repeating relational algebra queries can be done more efficiently than following this procedure via RC-hierarchical $\exists$-consistency, which incurs an exponential blowup in the size of the query.

\subsection{Non-repeating $\mathrm{RC}^{\exists}$}

There are subtle differences between non-repeating relational algebra and non-repeating $\mathrm{RC}^{\exists}$ that revolve around $\mathrm{RC}^{\exists}$ 's flexibility to allow disjunction and negation on sub-queries of different schemas. For instance, the non-repeating $\mathrm{RC}^{\exists}$ queries $S(x, y) \wedge \neg R(x)$ and $S(x, y) \wedge(R(x) \vee T(y))$ cannot be expressed in non-repeating relational algebra. Whereas the former query is tractable, the latter is \#P-hard: This means that non-repeating relational algebra cannot express both tractable and hard non-repeating $\mathrm{RC}^{\exists}$ queries.

For non-repeating $\mathrm{RC}^{\exists}$, the RC-hierarchical property alone does not characterise the tractable queries, even when we take away disjunction. Indeed, the $\mathrm{RC}^{\exists}$ query equivalent to the $1 \mathrm{RA}^{-}$query from Fig. 3, that is, $Q=\exists_{A} \exists_{B}[R(A) \wedge S(B) \wedge \neg(U(A) \wedge V(B))]$, does not satisfy the RC-hierarchical property since neither $A$ nor $B$ are root in the expression 
and they cannot be pushed further down. However, as for $1 \mathrm{RA}^{-}$queries, we can rewrite a non-repeating $\mathrm{RC}^{\exists}$ query $Q$ into an $\mathrm{RC}^{\exists}$ query $Q_{r}$ as outlined in Section $3.1 Q_{r}=$ $\exists_{A}[R(A) \wedge \neg U(A)] \wedge \exists_{B} S(B) \vee \exists_{A} R(A) \wedge \exists_{B}[S(B) \wedge \neg V(B)]$ for the above query $Q$, and then again $Q$ is tractable when $Q_{r}$ is RC-hierarchical and $\exists$-consistent.

\section{RELATED WORK}

Negation is a source of complexity already for databases with incomplete information and without probabilities [Abiteboul et al. 1991]. In probabilistic databases, the MystiQ system supports a limited class of NOT EXISTS queries Wang et al. 2008. A framework for the exact and approximate evaluation of full relational algebra queries (thus including negation) in probabilistic databases is part of SPROUT [Fink et al. 2011; Fink et al. 2013]. Further work looks at approximating queries with negation Khanna et al. 2011.

The dichotomy results of this article contribute to a succession of complexity results for queries on probabilistic databases: Starting from a first example of a \#P-hard query Grädel et al. 1998, polynomial-time/\#P-hard dichotomies have been established for non-repeating conjunctive queries Dalvi and Suciu 2004 and their ranking versions Olteanu and Wen 2012], and for unions of conjunctive queries (UCQs) Dalvi and Suciu 2012. Our result for $1 \mathrm{RA}^{-}$strictly generalises the dichotomy for non-repeating conjunctive queries. Whereas tractable $1 \mathrm{RA}^{-}$queries can be recognised efficiently via the hierarchical syntactic property, no such syntactic characterisation of tractable UCQs is known. Further tractability results are known for inequality joins Olteanu and Huang 2008; Olteanu and Huang 2009, Jha and Suciu 2012], and queries with aggregates and group-by clauses |Ré and Suciu 2009| Fink et al. 2012.

The closest in spirit to the proof techniques in this article are those connecting OBDDs with query tractability Olteanu and Huang 2008, Jha and Suciu 2013 and for the UCQ dichotomy result Dalvi and Suciu 2012. The algorithm for tractable UCQ queries translates them into relational calculus expressions that have root variables and satisfy properties similar to what we call canonicalised. Similar to root variables in our algorithm, the existence of separator variables for UCQs ensures that the annotations of the query expression are independent for different valuations of the separator variable. Our notion of $\exists$-consistency for queries with negation is inspired by the notion of inversion-freeness for UCQ queries.

Further related work, which has been developed independently of this work, is a dichotomy for a class of so-called Type-1 relational calculus queries with negation, that is, CNF formulas where each clause has at most two variables and each relational symbol is unary or binary Gribkoff et al. 2014a. The query languages considered in this article are incomparable with the Type-1 class. This work builds upon the UCQ dichotomy Dalvi and Suciu 2012 and as such it does not provide a syntactic characterisation of tractable queries.

The first connection between polysize OBDDs and tractable queries has been shown for hierarchical non-repeating conjunctive queries Olteanu and Huang 2008. For UCQ queries, the inversion-freenes property corresponds to polysize OBDDs [Jha and Suciu 2013. Queries with inequalities have been characterised in terms of their corresponding OBDDs Olteanu and Huang 2008, Olteanu and Huang 2009, Jha and Suciu 2012.

The problems of tractable query evaluation in probabilistic databases and of domainlifted inference for weighted first-order model counting den Broeck 2011] essentially coincide Gribkoff et al. 2014b. A common assumption in much existing work in probabilistic databases is that the probabilities of two tuples of a same relation may differ; this is referred to as the asymmetric probability case. The symmetric case, where all tuples of a relation have the same probability, is more common in lifted inference in AI. A number of complexity results have been recently shown for symmetric first-order model counting Beame et al. 2015. A promising direction of future research is combining the asymmetric and symmetric cases. 
The vast majority of hardness reductions in the above works are from the \#P-hard modelcounting problem for positive (2)DNF formulas Valiant 1979, Provan and Ball 1983. The complexity class \#P was originally defined by Valiant Valiant 1979. An overview of various topics in probabilistic databases has been compiled recently [Suciu et al. 2011].

\section{CONCLUSION}

This paper discusses a fundamental computational aspect of query processing in probabilistic databases, namely the classification of non-repeating conjunctive queries with negation and of quantified queries into tractable (polynomial-time) and intractable (\#P-hard) ones. The existence of an efficient recognition procedure for tractable queries allows a probabilistic query engine to switch between exact evaluation for tractable queries and approximate evaluation for intractable queries. A future challenge is understanding which extensions of the considered languages, e.g., with restricted union or repeating relation symbols, would still admit an efficient characterisation of tractable queries.

Acknowledgements. The authors are indebted to Dan Suciu for insightful discussions and to the anonymous reviewers for useful feedback on earlier drafts.

\section{REFERENCES}

Serge Abiteboul, Richard Hull, and Victor Vianu. 1995. Foundations of Databases. Addison-Wesley.

Serge Abiteboul, Paris Kanellakis, and Gösta Grahne. 1991. On the Representation and Querying of Sets of Possible Worlds. Theor. Comput. Sci. 78, 1 (1991), 158-187.

Vince Bárány, Balder ten Cate, and Martin Otto. 2012. Queries with Guarded Negation. PVLDB 5, 11 (2012), 1328-1339.

Paul Beame, Guy Van den Broeck, Eric Gribkoff, and Dan Suciu. 2015. Symmetric Weighted First-Order Model Counting. In Proc. PODS. 313-328.

Andrew Carlson, Justin Betteridge, Bryan Kisiel, Burr Settles, Estevam Junior Hruschka, and Tom Mitchell. 2010. Toward an Architecture for Never-Ending Language Learning. In AAAI.

Nilesh Dalvi and Dan Suciu. 2004. Efficient Query Evaluation on Probabilistic Databases. In VLDB. 864875 .

Nilesh Dalvi and Dan Suciu. 2007a. Efficient Query Evaluation on Probabilistic Databases. VLDB Journal 16, 4 (2007), 523-544.

Nilesh Dalvi and Dan Suciu. 2007b. Management of Probabilistic Data: Foundations and Challenges. In Proc. PODS. 1-12.

Nilesh N. Dalvi and Dan Suciu. 2012. The dichotomy of probabilistic inference for unions of conjunctive queries. J. ACM 59, 6 (2012), 30.

Guy Van den Broeck. 2011. On the Completeness of First-Order Knowledge Compilation for Lifted Probabilistic Inference. In NIPS. 1386-1394.

Xin Luna Dong, Evgeniy Gabrilovich, Geremy Heitz, Wilko Horn, Ni Lao, Kevin Murphy, Thomas Strohmann, Shaohua Sun, and Wei Zhang. 2014. Knowledge Vault: A Web-scale approach to probabilistic knowledge fusion. In SIGKDD. 601-610.

Robert Fink, Larisa Han, and Dan Olteanu. 2012. Aggregation in Probabilistic Databases via Knowledge Compilation. PVLDB 5, 5 (2012), 490-501.

Robert Fink, Jiewen Huang, and Dan Olteanu. 2013. Anytime Approximation in Probabilistic Databases. $V L D B$ J. 22, 6 (2013), 823-848.

Robert Fink and Dan Olteanu. 2014. A dichotomy for non-repeating queries with negation in probabilistic databases. In PODS. 144-155.

Robert Fink, Dan Olteanu, and Swaroop Rath. 2011. Providing Support for Full Relational Algebra Queries in Probabilistic Databases. In ICDE. 315-326.

Erich Grädel, Yuri Gurevich, and Colin Hirsch. 1998. The Complexity of Query Reliability. In Proc. PODS. $227-234$.

Todd J. Green, Grigoris Karvounarakis, and Val Tannen. 2007. Provenance Semirings. In PODS. 31-40.

Eric Gribkoff, Guy Van den Broeck, and Dan Suciu. 2014a. Understanding the Complexity of Lifted Inference and Asymmetric Weighted Model Counting. In UAI. 280-289.

Eric Gribkoff, Dan Suciu, and Guy Van den Broeck. 2014b. Lifted Probabilistic Inference: A Guide for the Database Researcher. IEEE Data Eng. Bull. 37, 3 (2014), 6-17. 
M. Grohe, Y. Gurevich, D. Leinders, N. Schweikardt, J. Tyszkiewicz, and J. V. den Bussche. 2009. Database query processing using finite cursor machines. Theory of Computing Syst. 44, 4 (2009), 533-560.

Jiewen Huang, Lyublena Antova, Christoph Koch, and Dan Olteanu. 2009. MayBMS: A Probabilistic Database Management System. In Proc. SIGMOD. 1071-1074.

T. Imielinski and W. Lipski. 1984. Incomplete information in relational databases. Journal of ACM 31, 4 (1984), 761-791.

Abhay Kumar Jha and Dan Suciu. 2012. On the tractability of query compilation and bounded treewidth. In Proc. ICDT. 249-261.

Abhay Kumar Jha and Dan Suciu. 2013. Knowledge Compilation Meets Database Theory: Compiling Queries to Decision Diagrams. Theory Comput. Syst. 52, 3 (2013), 403-440.

Sanjeev Khanna, Sudeepa Roy, and Val Tannen. 2011. Queries with Difference on Probabilistic Databases. PVLDB 4, 11 (2011), 1051-1062.

Paraschos Koutris and Dan Suciu. 2011. Parallel Evaluation of Conjunctive Queries. In Proc. PODS. 223234.

Dan Olteanu and Jiewen Huang. 2008. Using OBDDs for Efficient Query Evaluation on Probabilistic Databases. In Proc. SUM. 326-340.

Dan Olteanu and Jiewen Huang. 2009. Secondary-Storage Confidence Computation for Conjunctive Queries with Inequalities. In Proc. SIGMOD. 389-402.

Dan Olteanu and Hongkai Wen. 2012. Ranking Query Answers in Probabilistic Databases: Complexity and Efficient Algorithms. In ICDE. 282-293.

Dan Olteanu and Jakub Závodný. 2012. Factorised representations of query results: size bounds and readability. In Proc. ICDT. 285-298.

Dan Olteanu and Jakub Závodný. 2015. Size Bounds for Factorised Representations of Query Results. ACM Trans. Database Syst. 40, 1 (2015), 2.

J. Scott Provan and Michael O. Ball. 1983. The Complexity of Counting Cuts and of Computing the Probability that a Graph is Connected. SIAM J. Comput. 12, 4 (1983), 777-788.

Ralf Rantzau. 2004. Frequent Itemset Discovery with SQL Using Universal Quantification. In Database Support for Data Mining Applications. 194-213.

Sudhir Rao, Antonio Badia, and Dirk Van Gucht. 1996. Providing Better Support for a Class of Decision Support Queries. In Proc. SIGMOD. 217-227.

Christopher Ré and Dan Suciu. 2009. The Trichotomy of HAVING Queries on a Probabilistic Database. VLDB J. 18, 5 (2009), 1091-1116.

Omer Reingold. 2008. Undirected connectivity in log-space. J. ACM 55, 4 (2008), 17.

Dan Suciu, Dan Olteanu, Christopher Ré, and Christoph Koch. 2011. Probabilistic Databases. Morgan \& Claypool Publishers.

Leslie Valiant. 1979. The Complexity of Enumeration and Reliability Problems. SIAM J. Comput. 8 (1979), 410-421.

Ting-You Wang, Christopher Ré, and Dan Suciu. 2008. Implementing NOT EXISTS Predicates over a Probabilistic Database. In $Q D B / M U D .73-86$.

Ingo Wegener. 2004. BDDs-design, analysis, complexity, and applications. Discrete Applied Mathematics 138, 1-2 (2004), 229-251.

\section{A. RECOGNITION ALGORITHM FOR THE HIERARCHICAL PROPERTY}

The hierarchical property for $1 \mathrm{RA}^{-}$is given in Definition 1.2 The membership problem

Problem IsHierarchical: Input: $1 \mathrm{RA}^{-}$query $Q$

Output: "Yes" if $Q$ is hierarchical, "No" else

is the same problem as for conjunctive queries without negation Dalvi and Suciu 2007b], with the exception that IsHierarchical requires the computation of transitively joined attributes, while they are explicitly given in the case of conjunctive queries. Since the hierarchical property can be decided in $\mathrm{AC}_{0}$ Dalvi and Suciu 2007b and since deciding whether two attributes are transitively joined is in LOGSPACE, we may expect that IsHierarchical is in LOGSPACE.

Algorithm 3 gives an alternative, explicit LOGSPACE algorithm for deciding IsHierarchical. For each pair of variables $A, B$, the algorithm iterates over the relation symbols in 


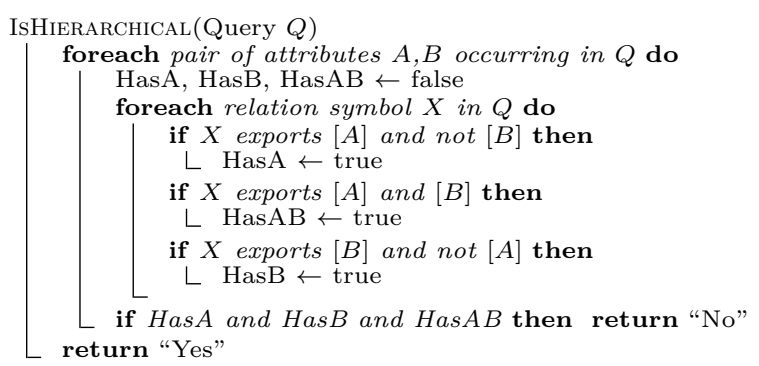

Algorithm 3: Algorithm to decide the hierarchical property for $1 \mathrm{RA}^{-}$queries.

$Q$ and indicates by three Boolean flags whenever one of the relations $R^{[A][\neg B]}, S^{[A][B]}$, or $T^{[\neg A][B]}$ has been found. This amounts to checking whether two attributes are transitively joined in $Q$, i.e. whether $A^{\prime} \in[A]$. The LOGSPACE complexity is due to the following argument. It uses a constant number of Boolean flags and a constant number of iterators over $Q$. Moreover, the transitive join condition $A^{\prime} \in[A]$ can be cast as the LOGSPACEproblem Reingold 2008] of checking whether $A$ and $A^{\prime}$ are connected in the undirected graph whose vertices are the attributes of $Q$ and which has an edge between $X$ and $Y$ if and only if $Q$ contains an operator $\bowtie_{\rho}$ with $(X=Y) \in \rho$ or an operator $-\rho$ with $(X \leftrightarrow Y) \in \rho$.

Corollary A.1 ([DAlVI AND SuCIu 2007B; ReINGOld 2008]). The decision problem IsHierarchical is in LOGSPACE.

Received November 2014; revised July 2015; accepted October 2015 